TRANSACTIONS OF THE

AMERICAN MATHEMATICAL SOCIETY

Volume 359, Number 3, March 2007, Pages 1205-1252

S 0002-9947(06)03904-3

Article electronically published on October 16, 2006

\title{
COX RINGS AND COMBINATORICS
}

\author{
FLORIAN BERCHTOLD AND JÜRGEN HAUSEN
}

\begin{abstract}
Given a variety $X$ with a finitely generated total coordinate ring, we describe basic geometric properties of $X$ in terms of certain combinatorial structures living in the divisor class group of $X$. For example, we describe the singularities, we calculate the ample cone, and we give simple Fano criteria. As we show by means of several examples, the results allow explicit computations. As immediate applications we obtain an effective version of the Kleiman-Chevalley quasiprojectivity criterion, and the following observation on surfaces: a normal complete surface with finitely generated total coordinate ring is projective if and only if any two of its non-factorial singularities admit a common affine neighbourhood.
\end{abstract}

\section{INTRODUCTION}

The remarkable success of Cox's homogeneous coordinate ring in toric geometry [8] has stimulated many authors to study related rings for more general varieties; see [1], 3], 99, [16] and [17]. Here we consider the version discussed in [9]: let $X$ be a normal variety with only constant invertible global functions and finitely generated free divisor class group $\mathrm{Cl}(X)$. Choose a subgroup $K$ of the group of Weil divisors mapping isomorphically onto $\mathrm{Cl}(X)$. Then the modules of global sections fit together to the total coordinate ring:

$$
\mathcal{R}(X):=\bigoplus_{D \in K} \Gamma(X, \mathcal{O}(D)) .
$$

For a toric variety $X$, the total coordinate ring is a polynomial ring [8]. In general, it turns out to be a factorial ring after all; see [3] and, more generally, 9]. The present paper is devoted to the study of varieties $X$ with a finitely generated total coordinate ring. For example, finite generation of $\mathcal{R}(X)$ is known to hold for smooth Fano varieties of dimension at most three [17] and for smooth, unirational varieties with a complexity one group action [21].

In [17, Keel and $\mathrm{Hu}$ characterized, for $\mathbb{Q}$-factorial projective varieties $X$, finite generation of $\mathcal{R}(X)$ in terms of Mori Theory. Moreover, they observed a connection to GIT: if $X$ is $\mathbb{Q}$-factorial projective with $\mathcal{R}(X)$ finitely generated, then $X$ is the geometric quotient of a set $\widehat{X} \subset \bar{X}$ of semistable points of the affine variety $\bar{X}=\operatorname{Spec}(\mathcal{R}(X))$ with respect to the natural action of the Neron Severi torus. The small modifications of $X$ are reflected in the associated GIT chamber structure, and the various cones of divisors arising in this context, such as the effective cone, the closure of the ample cone, and the Mori cone are all polyhedral.

Received by the editors August 23, 2004 and, in revised form, December 10, 2004.

2000 Mathematics Subject Classification. Primary 14C20, 14J45, 14J70, 14M20, 14M25, 14Q15. 
In the present paper, we extend the picture developed in [17] to certain normal, not necessarily (quasi)projective varieties $X$. The main aim then is to provide a framework that allows explicit descriptions of geometric properties of $X$ in terms of combinatorial data living in the divisor class group $\mathrm{Cl}(X)$. The approach generalizes the description of toric varieties in terms of "bunches of cones" presented in 4. As we shall see, it allows a systematic treatment of many non-toric examples, and thus enlarges the "testing ground of algebraic geometry" provided by the toric varieties. For example, many torus quotients of Grassmannians fit into our framework.

We now present the results in a little more detail. Similar to [4, we start with a combinatorial input datum, and then obtain a variety by means of a certain multigraded homogeneous spectrum construction. The input is what we call a bunched ring $(R, \mathfrak{F}, \Phi)$; the precise definition is given in Section 2 , For the moment, it suffices to know that $R$ is a finitely generated factorial algebra, graded by a lattice $K$, that $\mathfrak{F}$ is a system of $K$-homogeneous prime generators $f_{1}, \ldots, f_{r}$ of $R$, and that $\Phi$ is a certain finite collection of pairwise overlapping polyhedral cones in $K \otimes_{\mathbb{Z}} \mathbb{Q}$, each of which is generated by the degrees of some of the $f_{i}$.

The basic construction then associates to the bunched ring $(R, \mathfrak{F}, \Phi)$ a normal variety $X=X(R, \mathfrak{F}, \Phi)$ of dimension $\operatorname{dim}(R)-\operatorname{rank}(K)$ having divisor class group $K$ and total coordinate $\operatorname{ring} R$; see Section 2. Geometrically, $X$ is a good quotient space of an open subset $\widehat{X} \subset \bar{X}$ of $\bar{X}:=\operatorname{Spec}(\mathcal{R}(X))$ by the torus action arising from the $K$-grading. The variety $X$ admits embeddings into toric varieties, and thus inherits the $A_{2}$-property, i.e., any pair $x, x^{\prime} \in X$ has a common affine neighbourhood; compare [31. In fact $X$ is even $A_{2}$-maximal in the following sense: for any open embedding $X \subset X^{\prime}$ into an $A_{2}$-variety $X^{\prime}$ such that $X^{\prime} \backslash X$ is of codimension at least two, we have $X=X^{\prime}$. Theorem 4.2 shows the converse.

Theorem. Every $A_{2}$-maximal (e.g., projective) normal variety with free finitely generated divisor class group and finitely generated total coordinate ring arises from a bunched ring.

As indicated, a central aim is to describe geometric properties of the variety $X$ arising from a bunched ring $(R, \mathfrak{F}, \Phi)$ in terms of the defining data. A first observation is that the system $\mathfrak{F}$ provides a decomposition of the variety $X$. The generators $f_{i} \in \mathfrak{F}$ induce certain divisors $D_{X}^{i} \subset X$ (see Section 3 ), and for any face $\gamma_{0}$ of the positive orthant $\gamma:=\mathbb{Q}_{\geq 0}^{r}$, one may consider the locally closed stratum

$$
X\left(\gamma_{0}\right):=\bigcap_{e_{i} \notin \gamma_{0}} D_{X}^{i} \backslash \bigcup_{e_{i} \in \gamma_{0}} D_{X}^{i} \subset X .
$$

We call a face $\gamma_{0} \preceq \gamma$ relevant if the corresponding $X\left(\gamma_{0}\right)$ is non-empty, and we denote the set of relevant faces by $\operatorname{rlv}(\Phi)$. Thus, $X$ is decomposed into the $X\left(\gamma_{0}\right)$, where $\gamma_{0}$ runs through the relevant faces. Moreover, we need the linear map $Q: \mathbb{Z}^{r} \rightarrow K$ sending the canonical base vector $e_{i}$ to the degree of $f_{i}$. A first result shows that the decomposition of $X$ is equisingular in a raw sense; see Theorem 5.4 .

Theorem. Consider a stratum $X\left(\gamma_{0}\right) \subset X$ and a point $x \in X\left(\gamma_{0}\right)$. Then $X$ is $\mathbb{Q}$ factorial (factorial) at $x$ if and only if $Q\left(\gamma_{0}\right)$ is of full dimension $\left(Q\right.$ maps $\operatorname{lin}\left(\gamma_{0}\right) \cap E$ onto $K)$.

In general, there is no way to describe the smooth points in combinatorial terms. Nevertheless, if the ring $R$ is "regular enough" in the sense that $\widehat{X}$ is smooth, then the factorial points of $X$ are even smooth; see Proposition 5.6. This criterion applies 
to all our examples. Furthermore, in this setting, the singularities of $X$ are at most rational.

If we pick out the set theoretically minimal cones from the relevant faces, we arrive at the covering collection $\operatorname{cov}(\Phi) \subset \operatorname{rlv}(\Phi)$. In terms of this covering collection, we determine in Proposition 7.1 the Picard group.

Theorem. In the divisor class group $K=\mathrm{Cl}(X)$, the Picard group of $X$ is given by

$$
\operatorname{Pic}(X)=\bigcap_{\gamma_{0} \in \operatorname{cov}(\Phi)} Q\left(\operatorname{lin}\left(\gamma_{0}\right) \cap E\right) .
$$

Moreover, in Proposition 7.2 and Theorem 7.3, we describe the effective cone, the moving cone, the semiample cone and, finally, the ample cone. The statement generalizes corresponding results on toric varieties; compare [4] and [16].

Theorem. In the divisor class group $K=\mathrm{Cl}(X)$, we have the following descriptions:

$$
\begin{array}{cr}
\operatorname{Eff}(X)=Q(\gamma), & \operatorname{Mov}(X)=\bigcap_{\gamma_{0} \text { facet of } \gamma} Q\left(\gamma_{0}\right), \\
\operatorname{Sample}(X)=\bigcap_{\tau \in \Phi} \tau, & \operatorname{Ample}(X)=\bigcap_{\tau \in \Phi} \tau^{\circ} .
\end{array}
$$

An immediate application is the description of the Mori cone given in Corollary 7.5. As a further application, we give in Section 8 an effective KleimanChevalley Criterion, which in the case of varieties with finitely generated coordinate ring sharpens considerations presented in 23]: suppose that $X$ is $\mathbb{Q}$-factorial and complete, and let $k$ be the rank of the divisor class group of $X$. Then $X$ is projective if and only if any $k$ points of $X$ admit a common affine neighbourhood in $X$.

If the variety $X$ is sort of an "intrinsic complete intersection", then one can determine its canonical divisor class in $K=\mathrm{Cl}(X)$, and thus obtains Fano criteria; see Proposition 8.5 and Corollary 8.7

Theorem. Let $d:=r-\operatorname{dim}(X)-\operatorname{dim}(K)$, and suppose that $g_{1}, \ldots, g_{d}$ are $K$ homogeneous generators for the relations between $f_{1}, \ldots, f_{r}$. Then

$$
\sum \operatorname{deg}\left(g_{j}\right)-\sum \operatorname{deg}\left(f_{i}\right)
$$

is the canonical divisor class of $X$ in $K=\mathrm{Cl}(X)$. Moreover, $X$ is a Fano variety if and only if

$$
\sum \operatorname{deg}\left(f_{i}\right)-\sum \operatorname{deg}\left(g_{j}\right) \in \bigcap_{\tau \in \Phi} \tau^{\circ} \cap \bigcap_{\gamma_{0} \in \operatorname{cov}(\Phi)} Q\left(\operatorname{lin}\left(\gamma_{0}\right) \cap E\right) .
$$

The language of bunched rings can be used as well to produce and study (new) examples. To indicate how this may run, we consider in Section 9 the case of coordinate rings defined by a single relation. In that situation, the collection of relevant faces can be easily and explicitly determined in terms of the coefficients of the defining equation. For the case of a divisor class group of rank two and coordinate rings defined by a quadric, we provide a classification extending Kleinschmidt's classification of toric varieties with few invariant divisors [20]; see Theorem 9.9 , 
Theorem. The smooth intrinsic quadrics of full rank with divisor class group $\mathbb{Z}^{2}$ and only constant functions arise from bunched rings $(R, \mathfrak{F}, \Phi)$ with $\Phi$ given by a figure as follows:
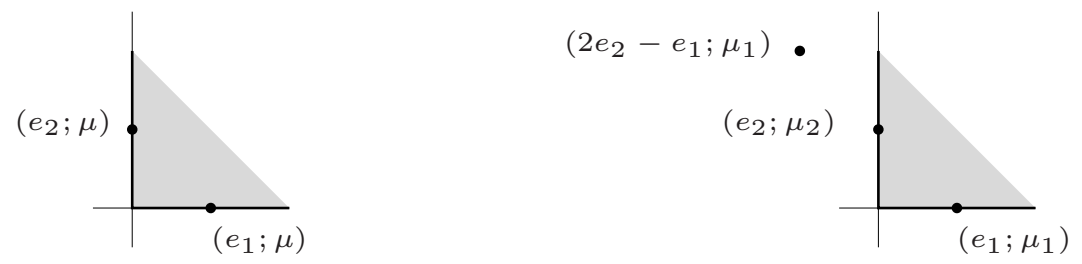

All these varieties are projective. They are Fano if and only if $\Phi$ arises from a figure on the left-hand side. Moreover, different figures belong to non-isomorphic varieties.

In these pictures, the thick points indicate the degrees of the generators $f_{i} \in \mathfrak{F}$; note that these degrees may occur with multiplicity, which is indicated by the $\mu$ 's. The shadowed cones are the elements of the respective "F-bunches" $\Phi$; in the above situation, each of these $\Phi$ consists of a single cone.

The proof is elementary: it is an interplay of the combinatorics of bunches with the symmetry conditions imposed by the defining quadric. Classification problems for class groups of higher rank, and defining equations of higher degree(s), seem to pose new, elementary, but non-trivial combinatorial challenges.

Finally, we take a closer look at complete surfaces $X$ with finitely generated total coordinate ring $R:=\mathcal{R}(X)$. First we discuss general properties. It turns out that $X$ is already determined by $R$; see Proposition 11.1 Thus, in the description $X=X(R, \mathfrak{F}, \Phi)$, there is a certain freedom of choice for $\mathfrak{F}$ and $\Phi$. This is the key to the following observation; see Corollary 11.3.

Proposition. Let $X$ be a normal complete surface with free finitely generated divisor class group and finitely generated total coordinate ring. If any two non-factorial singularities of $X$ admit a common affine neighbourhood in $X$, then $X$ is projective.

Then we consider some explicit examples, and indicate how the results presented before can be used for explicit computations. We study a certain two-dimensional Mumford quotient of the Grassmannian $G(2,4)$ and determine its singularities, the Picard group, the (semi-)ample cone, and the canonical divisor. Moreover, we consider the minimal resolution of the $E_{6}$ cubic surface, studied recently by Hasset and Tschinkel [16].

We would like to thank the referee for his careful reading, and for a long list of very helpful comments and valuable suggestions for improvement.

\section{Bunches AND TORIC VARIETIES}

The combinatorial description of toric varieties in terms of bunches presented in [4] is an essential ingredient of the present paper. In this section we recall the necessary concepts. It should be mentioned that we only need a special case: here the word "bunch" will refer to what we called in 44 a "free standard bunch".

In the entire paper, by a variety we mean an integral scheme over an algebraically closed field $\mathbb{K}$ of characteristic zero, and the word "point" refers to a closed point. Concerning toric varieties, we assume the reader to be familiar with the basic theory; see for example [11]. 
To begin, let us fix terminology and notation from convex geometry. A lattice is a finitely generated free abelian group. Given a lattice $N$, we denote by $N_{\mathbb{Q}}:=N \otimes_{\mathbb{Z}} \mathbb{Q}$ the associated rational vector space. For a homomorphism $P: F \rightarrow N$ of lattices, the induced map $F_{\mathbb{Q}} \rightarrow N_{\mathbb{Q}}$ is again denoted by $P$.

By a cone in a lattice $N$ we mean a (not necessarily strictly) convex polyhedral cone $\sigma \subset N_{\mathbb{Q}}$. The relative interior of a cone $\sigma$ is denoted by $\sigma^{\circ}$, its dual cone by $\sigma^{\vee}$, and its orthogonal space by $\sigma^{\perp}$. If $\sigma_{0}$ is a face of a cone $\sigma$, then we write $\sigma_{0} \preceq \sigma$. A cone is called simplicial if it is generated by linearly independent vectors, and it is called regular if it is generated by part of a lattice basis.

A fan in a lattice $N$ is a finite collection $\Delta$ of strictly convex cones in $N$ such that for $\sigma \in \Delta$ every $\sigma_{0} \preceq \sigma$ also belongs to $\Delta$, and for any two $\sigma_{1}, \sigma_{2} \in \Delta$ we have $\sigma_{1} \cap \sigma_{2} \preceq \sigma_{i}$. Given a fan $\Delta$, we denote by $\Delta^{\max }$ the set of its maximal cones and by $\Delta^{(k)}$ the set of its $k$-dimensional cones.

Let us now recall the basic concepts and constructions from 44. By a projected cone here we mean a pair $(E \stackrel{Q}{\longrightarrow} K, \gamma)$, where $Q: E \rightarrow K$ is a surjection of lattices, and $\gamma \subset E_{\mathbb{Q}}$ is a regular polyhedral convex cone of full dimension. A projected face is the image $Q\left(\gamma_{0}\right)$ of a face $\gamma_{0} \preceq \gamma$.

Definition 1.1. A bunch in a projected cone $(E \stackrel{Q}{\longrightarrow} K, \gamma)$ is a non-empty collection $\Theta$ of projected faces with the following properties:

(i) A projected face $\sigma$ belongs to $\Theta$ if and only if $\emptyset \neq \sigma^{\circ} \cap \tau^{\circ} \neq \tau^{\circ}$ holds for all $\tau \in \Theta$ with $\tau \neq \sigma$.

(ii) For each facet $\gamma_{0} \preceq \gamma$, the images of the primitive generators of $\gamma_{0}$ generate the lattice $K$, and there is a $\tau \in \Theta$ with $\tau^{\circ} \subset Q\left(\gamma_{0}\right)^{\circ}$.

Note that a collection $\Theta$ of projected faces satisfies Definition 1.1(i) if and only if any two cones of $\Theta$ overlap, $\Theta$ is irredundant in the sense that no two cones of $\Theta$ are strictly contained one in the other, and $\Theta$ is maximal in the sense that any projected face that overlaps with each cone of $\Theta$ must contain a cone of $\Theta$.

The basic construction of 4 associates to a given bunch $\Theta$ living in a projected cone $(E \stackrel{Q}{\longrightarrow} K, \gamma)$ a toric variety $Z_{\Theta}$. This construction is purely combinatorial; using a certain Gale duality, one transforms the bunch $\Theta$ into a fan $\Delta$ and thus obtains a toric variety.

Here comes the exact procedure: consider the dual projected cone $(F \stackrel{P}{\longrightarrow} N, \delta)$; that means that we have $F=\operatorname{Hom}(E, \mathbb{Z})$, the map $P: F \rightarrow N$ is dual to the inclusion $M \rightarrow E$ of $M:=\operatorname{ker}(Q)$, and $\delta:=\gamma^{\vee}$ is the dual cone of $\gamma$. Then we have the face correspondence

$$
\operatorname{faces}(\gamma) \rightarrow \operatorname{faces}(\delta), \quad \gamma_{0} \mapsto \gamma_{0}^{*}:=\gamma_{0}^{\perp} \cap \delta .
$$

The covering collection $\operatorname{cov}(\Theta)$ of the bunch $\Theta$ is the set of all faces $\gamma_{0} \preceq \gamma$ that are minimal with respect to the property that $Q\left(\gamma_{0}\right)$ contains a cone of $\Theta$. Via the face correspondence, we define a fan in $F$ :

$$
\widehat{\Delta}:=\left\{\delta_{0} ; \delta_{0} \preceq \gamma_{0}^{*} \text { for some } \gamma_{0} \in \operatorname{cov}(\Theta)\right\} .
$$

Property 1.1 (i) implies that $\widehat{\Delta}$ is a maximal projectable fan in $(F \stackrel{P}{\longrightarrow} N, \delta)$ in the sense that it consists of faces of $\delta$ and is maximal with respect to the following property: any two maximal cones of $\widehat{\Delta}$ can be separated by linear forms that are invariant with respect to $L:=\operatorname{Hom}(K, \mathbb{Z})$, i.e., that vanish on $L$. 
As a consequence of property 1.1](ii), one obtains that the projected faces $P\left(\delta_{0}\right)$, where $\delta_{0} \in \widehat{\Delta}^{\max }$, are strictly convex. Thus, the $P\left(\delta_{0}\right)$ are the maximal cones of a fan $\Delta$ in the lattice $N$, the quotient fan of $\widehat{\Delta}$. In conclusion, transforming $\Theta$ into $\Delta$ runs according to the following scheme:

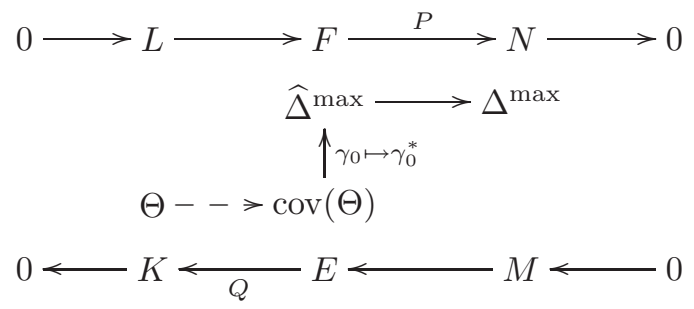

where the two exact sequences are dual to each other, and the dashed arrow indicates the passage to the covering collection. Note that this procedure may also be reversed. In fact, in [4, Sec. 5] it is shown that it provides a one-to-one correspondence between bunches and maximal projectable fans.

Definition 1.2. The toric variety $Z_{\Theta}$ associated to the bunch $\Theta$ is the toric variety arising from the fan $\Delta$ in the lattice $N$.

In the sequel, we shall often omit the subscript $\Theta$ and just speak of the toric variety $Z$ associated to the bunch $\Theta$. Recall from [4, Theorem 7.4 and Prop. 10.6] that $Z_{\Theta}$ is non-degenerate in the sense that $\mathcal{O}^{*}\left(Z_{\Theta}\right)=\mathbb{K}^{*}$ holds, and that $Z_{\Theta}$ has free divisor class group, canonically isomorphic to $K$.

We need a description of the orbit stratification of the toric variety $Z$ associated to $\Theta$. First recall from 11 that the cones of $\Delta$ correspond to the orbits of the big torus $T_{Z} \subset Z$ via $\sigma \mapsto T_{Z} \cdot z_{\sigma}$. Here $z_{\sigma} \in Z$ denotes the distinguished point of the affine chart $Z_{\sigma}=\operatorname{Spec}(\mathbb{K}[\sigma \cap N])$ of $Z$; it is determined by

$$
\chi^{u}\left(z_{\sigma}\right)=1 \quad \text { if } u \in \sigma^{\perp}, \quad \chi^{u}\left(z_{\sigma}\right)=0 \quad \text { if } u \in \sigma^{\vee} \backslash \sigma^{\perp} .
$$

We want to translate this into the language of bunches. Call a face $\gamma_{0} \preceq \gamma$ relevant if $Q\left(\gamma_{0}\right)^{\circ} \supset \tau^{\circ}$ holds for some $\tau \in \Theta$, and denote by $\operatorname{rlv}(\Theta)$ the collection of relevant faces. Then [4, Prop. 5.11] tells us that the cones of the fan $\Delta$ are exactly the images $P\left(\gamma_{0}^{*}\right)$, where $\gamma_{0}^{*} \in \operatorname{rlv}(\Theta)$. This gives the desired description of the orbit stratification.

Proposition 1.3. There is a canonical bijection

$$
\operatorname{rlv}(\Theta) \rightarrow\left\{T_{Z} \text {-orbits of } Z\right\}, \quad \gamma_{0} \mapsto Z\left(\gamma_{0}\right):=T_{Z} \cdot z_{P\left(\gamma_{0}^{*}\right)}
$$

We shall frequently need the fact that the toric morphism $p_{Z}: \widehat{Z} \rightarrow Z$ arising from the map $P: F \rightarrow N$ of the fans $\widehat{\Delta}$ and $\Delta$ is a Cox construction. That means that we have:

Proposition 1.4. Every ray of $\delta$ occurs in $\widehat{\Delta}$, and the map $P: F \rightarrow N$ induces bijections $\widehat{\Delta}^{\max } \rightarrow \Delta^{\max }$ and $\widehat{\Delta}^{(1)} \rightarrow \Delta^{(1)}$. Moreover, $P$ maps the primitive generator of any ray $\widehat{\varrho} \in \widehat{\Delta}^{(1)}$ to a primitive lattice vector in $N$.

Let us also interpret this geometrically. Consider the affine toric variety $\bar{Z}:=$ $\operatorname{Spec}(\mathbb{K}[\gamma \cap E])$ with its big torus $T_{\bar{Z}}:=\operatorname{Spec}(\mathbb{K}[E])$. The $K$-grading of $\mathbb{K}[\gamma \cap E]$ defines actions of $T:=\operatorname{Spec}(\mathbb{K}[K])$ on $\bar{Z}$ and on the $T_{\bar{Z}}$-invariant open subset 
$\widehat{Z} \subset \bar{Z}$ corresponding to the fan $\widehat{\Delta}$. The geometric version of Proposition 1.4 is the following.

Proposition 1.5. (i) The toric morphism $p_{Z}: \widehat{Z} \rightarrow Z$ is a good quotient for the $T$-action. That means that it is affine, $T$-invariant and defines an isomorphism $\mathcal{O}_{Z} \rightarrow p_{*}\left(\mathcal{O}_{\widehat{Z}}\right)^{T}$.

(ii) Let $W_{Z} \subset Z$ and $W_{\bar{Z}} \subset \bar{Z}$ be the unions of all at most one codimensional orbits of the respective big tori. Then $W_{\bar{Z}}=p_{Z}^{-1}\left(W_{Z}\right)$ and $W_{\bar{Z}} \subset \widehat{Z}$ hold, and $T$ acts freely on $W_{\bar{Z}}$.

For later purposes, we note here an elementary observation on existence of polytopal fans with a prescribed set of rays; compare also [16, Prop. 3.4].

Lemma 1.6. Let $N$ be a lattice, and let $v_{1}, \ldots, v_{r} \in N$ be vectors generating $N$ as a lattice and $N_{\mathbb{Q}}$ as a cone. Then there is a polytopal fan $\Delta$ in $N$ having precisely $\mathbb{Q}_{\geq 0} v_{1}, \ldots, \mathbb{Q}_{\geq 0} v_{r}$ as its rays.

Proof. First note that there is a polytopal fan $\Delta_{0}$ in $N$ the rays of which form a subset of the prescribed choice, say $\mathbb{Q}_{\geq 0} v_{1}, \ldots, \mathbb{Q}_{\geq 0} v_{s}$ for some $s \leq r$; see e.g. [27. Then we may construct a new polytopal fan $\Delta_{1}$ from $\Delta_{0}$ by inserting the ray $\mathbb{Q}_{>0} v_{s+1}$ via stellar subdivision. Iterating this process, we finally arrive at the desired fan.

We shall also need the bunch theoretical analogue of the above observation. It is the following.

Lemma 1.7. Let $(E \stackrel{Q}{\longrightarrow} K, \gamma)$ be a projected cone such that $Q(\gamma)$ is strictly convex, and let $\gamma_{1}, \ldots, \gamma_{r}$ be the facets of $\gamma$. Suppose that each $Q\left(\operatorname{lin}\left(\gamma_{i}\right) \cap E\right)$ generates $K$ as a lattice, and that for any two $\gamma_{i}, \gamma_{j}$ we have $Q\left(\gamma_{i}\right)^{\circ} \cap Q\left(\gamma_{j}\right)^{\circ} \neq \emptyset$. Then $Q\left(\gamma_{1}\right)^{\circ} \cap \ldots \cap Q\left(\gamma_{r}\right)^{\circ} \neq \emptyset$ holds.

Proof. First consider the dual projected cone $(F \stackrel{P}{\longrightarrow} N, \delta)$. Then the images $v_{1}, \ldots, v_{r} \in N$ of the primitive generators of $\delta$ are as in Lemma 1.6. Hence, we find a polytopal fan $\Delta$ in $N$ having precisely $\mathbb{Q}_{\geq 0} v_{1}, \ldots, \mathbb{Q}_{\geq 0} v_{r}$ as its rays.

Now consider the bunch $\Theta$ in $(E \stackrel{Q}{\longrightarrow} K, \gamma)$ corresponding to $\Delta$. According to 4, Thm. 10.2], the intersection over all relative interiors $\tau^{\circ}$, where $\tau \in \Theta$, is nonempty. Since every $\mathbb{Q}\left(\gamma_{i}\right)^{\circ}$ contains some $\tau^{\circ}$, the assertion follows.

We conclude this section with a few words towards the visualization of bunches. Look at the following example: let $K:=\mathbb{Z}$, and consider the elements $w_{1}=2$, $w_{2}=4$, and $w_{3}=5$ in $K$. Attach to each $w_{i}$ a multiplicity $\mu_{i}$ by setting $\mu_{1}:=1$, $\mu_{2}:=3$, and $\mu_{3}:=2$. Finally, set $\Theta:=\left\{\mathbb{Q}_{\geq 0}\right\}$. Then these data fit into the following figure:

$$
(2 ; 1) \quad(4 ; 3) \quad(5 ; 2)
$$

We obtain an associated projected cone $(E \stackrel{Q}{\longrightarrow} K, \gamma)$ by taking the lattice $E:=$ $\mathbb{Z}^{6}$, the map $Q: E \rightarrow K$, defined by $e_{1} \mapsto 2, e_{2}, e_{3}, e_{4} \mapsto 4$, and $e_{5}, e_{6} \mapsto 5$, and finally, the cone $\gamma:=\mathbb{Q}_{>0}^{6}$. The associated toric variety turns out to be the weighted projective space $\mathbb{P}(2,4,4,4,5,5)$.

This principle also allows us to draw pictures in general: any bunch can represented by a set $\left\{\left(w_{i} ; \mu_{i}\right) ; i \in I\right\}$ of lattice vectors $w_{i}$ carrying multiplicities $\mu_{i}$, and 
a collection $\Theta$ of cones, each of which is generated by some of the $w_{i}$, such that the whole setup satisfies conditions analogous to those of Definition 1.1 .

\section{BUNCHED RINGS AND VARIETIES}

In this section, we first put the concept of a bunch into a more general framework, and we introduce the notion of a bunched ring. Then the central construction of the paper is presented: a generalized multigraded proj-construction that associates to any bunched ring a variety coming along with a certain stratification.

Let us fix the setup of the section. $R$ is a factorial, finitely generated $\mathbb{K}$-algebra, faithfully graded by some lattice $K \cong \mathbb{Z}^{k}$. Here, faithfully graded means that $K$ is generated as a lattice by the degrees $w \in K$ admitting non-trivial homogeneous elements $f \in R_{w}$.

Moreover, $\mathfrak{F}=\left\{f_{1}, \ldots, f_{r}\right\} \subset R$ is a system of homogeneous pairwise nonassociated non-zero prime elements generating $R$ as an algebra. Since we assume the grading to be faithful, the degrees $w_{i}:=\operatorname{deg}\left(f_{i}\right)$ generate the lattice $K$.

The projected cone $(E \stackrel{Q}{\longrightarrow} K, \gamma)$ associated to the system of generators $\mathfrak{F} \subset R$ consists of the surjection $Q$ of the lattices $E:=\mathbb{Z}^{r}$ and $K$ sending the $i$-th canonical base vector $e_{i}$ to $w_{i}$, and the cone $\gamma \subset E_{\mathbb{Q}}$ generated by $e_{1}, \ldots, e_{r}$.

Definition 2.1. Let $(E \stackrel{Q}{\longrightarrow} K, \gamma)$ denote the projected cone associated to $\mathfrak{F} \subset R$.

(i) We call $\gamma_{0} \preceq \gamma$ an $\mathfrak{F}$-face if the product over all $f_{i}$ with $e_{i} \in \gamma_{0}$ does not belong to the ideal $\sqrt{\left\langle f_{j} ; e_{j} \notin \gamma_{0}\right\rangle} \subset R$.

(ii) By an $\mathfrak{F}$-bunch we mean a non-empty collection $\Phi$ of projected $\mathfrak{F}$-faces such that

- a projected $\mathfrak{F}$-face $\tau$ belongs to $\Phi$ if and only if for each $\tau \neq \sigma \in \Phi$ we have $\emptyset \neq \tau^{\circ} \cap \sigma^{\circ} \neq \sigma^{\circ}$,

- for each facet $\gamma_{0} \preceq \gamma$, the image $Q\left(\gamma_{0} \cap E\right)$ generates the lattice $K$, and there is a $\tau \in \Phi$ such that $Q\left(\gamma_{0}\right)^{\circ} \supset \tau^{\circ}$ holds.

If we want to specify these data, then we also speak of the $\mathfrak{F}$-bunch in the projected cone $(E \stackrel{Q}{\longrightarrow} K, \gamma)$. We shall frequently use the following more geometric characterization of the $\mathfrak{F}$-faces.

Remark 2.2. Let $\bar{X}:=\operatorname{Spec}(R)$. Then a face $\gamma_{0} \preceq \gamma$ is an $\mathfrak{F}$-face if and only if there is a point $x \in \bar{X}$ satisfying $e_{i} \in \gamma_{0} \Leftrightarrow f_{i}(x) \neq 0$.

We come to the central definition of the paper: the notion of a bunched ring. As we shall see, this generalizes the usual notion of a bunch in a projected cone.

Definition 2.3. A bunched ring is a triple $(R, \mathfrak{F}, \Phi)$, where

(i) $R$ is a finitely generated factorial $\mathbb{K}$-algebra with $R^{*}=\mathbb{K}^{*}$, faithfully graded by a finite-dimensional lattice $K$,

(ii) $\mathfrak{F}=\left\{f_{1}, \ldots, f_{r}\right\}$ is a system of homogeneous pairwise non-associated nonzero prime elements generating $R$ as an algebra,

(iii) $\Phi$ is an $\mathfrak{F}$-bunch in the projected cone $(E \stackrel{Q}{\longrightarrow} K, \gamma)$ associated to $\mathfrak{F}$.

Let us illustrate this definition by means of some examples. The second one shows how the usual notion of a bunch discussed in the previous section fits into the framework of $\mathfrak{F}$-bunches and bunched rings. 
Example 2.4 (Trivially bunched rings). Any finitely generated factorial $\mathbb{K}$-algebra $R$ with $R^{*}=\mathbb{K}^{*}$ can be made trivially into a bunched ring: take the trivial grading by $K=\{0\}$, any system $\mathfrak{F}=\left\{f_{1}, \ldots, f_{r}\right\}$ of pairwise non-associated prime generators, and the trivial $\mathfrak{F}$-bunch $\Phi=\{0\}$ in the projected cone $(E \stackrel{Q}{\longrightarrow} K, \gamma)$, where $E:=\mathbb{Z}^{r}$, and $\gamma \subset E_{\mathbb{Q}}$ is the positive orthant.

Example 2.5 (Bunched polynomial rings). Let $R=\mathbb{K}\left[T_{1}, \ldots, T_{r}\right]$. Suppose that each variable $T_{i}$ is homogeneous with respect to the $K$-grading of $R$, and let $\mathfrak{F}:=$ $\left\{T_{1}, \ldots, T_{r}\right\}$. Then any bunch $\Theta$ in the projected cone associated to $\mathfrak{F}$ is an $\mathfrak{F}$-bunch, and thus defines a bunched ring $(R, \mathfrak{F}, \Theta)$.

Example 2.6 (Homogeneous coordinates of the Grassmannian $G(2,4)$ ). Take the lattice $K:=\mathbb{Z}$, and consider the $K$-graded factorial ring defined by

$$
R:=\mathbb{K}\left[T_{1}, \ldots, T_{6}\right] /\left\langle T_{1} T_{6}-T_{2} T_{5}+T_{3} T_{4}\right\rangle, \quad \operatorname{deg}\left(T_{i}\right):=1,1 \leq i \leq 6 .
$$

The projected cone $(E \stackrel{Q}{\longrightarrow} K, \gamma)$ associated to the system $\mathfrak{F}:=\left\{T_{1}, \ldots, T_{6}\right\}$ of generators is given by $E:=\mathbb{Z}^{6}$, the map $Q: E \rightarrow K$ sending $e_{i}$ to 1 , and the cone $\gamma:=\mathbb{Q}_{\geq 0}^{6}$. Setting $\Phi:=\left\{\mathbb{Q}_{\geq 0}\right\}$, we obtain a bunched ring $(R, \mathfrak{F}, \Phi)$.

Let us now present the basic construction of the paper. As mentioned, it associates to a given bunched ring $(R, \mathfrak{F}, \Phi)$ a variety $X(R, \mathfrak{F}, \Phi)$. The first step is to generalize the concepts of relevant faces and the covering collection defined in Section 1

Definition 2.7. Let $\Phi$ be an $\mathfrak{F}$-bunch in the projected cone $(E \stackrel{Q}{\longrightarrow} K, \gamma)$.

(i) The collection $\operatorname{rlv}(\Phi)$ of relevant faces consists of those $\mathfrak{F}$-faces $\gamma_{0} \preceq \gamma$ such that $Q\left(\gamma_{0}\right)^{\circ} \supset \tau^{\circ}$ holds for some $\tau \in \Phi$.

(ii) The covering collection $\operatorname{cov}(\Phi)$ of $\Phi$ is the set of all minimal cones of $\operatorname{rlv}(\Phi)$.

Note that this indeed gives nothing new for a usual bunch when we regard it as an $\mathfrak{F}$-bunch in the sense of Example 2.5

Remark 2.8. Let $\Phi$ be an $\mathfrak{F}$-bunch with associated projected cone $(E \stackrel{Q}{\longrightarrow} K, \gamma)$, and let $\gamma_{0}, \gamma_{1} \in \operatorname{cov}(\Phi)$. Then $Q\left(\gamma_{0}\right)^{\circ} \cap Q\left(\gamma_{1}\right)^{\circ} \neq \emptyset$.

Let $(R, \mathfrak{F}, \Phi)$ be a bunched ring, and let $(E \stackrel{Q}{\longrightarrow} K, \gamma)$ be the projected cone associated to $\mathfrak{F}$. To construct the variety $X(R, \mathfrak{F}, \Phi)$, we consider the affine variety $\bar{X}:=\operatorname{Spec}(R)$, the action of the torus $T:=\operatorname{Spec}(\mathbb{K}[K])$ on $\bar{X}$ induced by the $K$-grading of $R$, and the invariant open subset

$$
\widehat{X}=\bigcup_{\gamma_{0} \in \operatorname{cov}(\Phi)} \bar{X}_{\gamma_{0}}, \quad \text { where } \bar{X}_{\gamma_{0}}=\bar{X}_{f^{u}} \text { for some } u \in \gamma_{0}^{\circ} .
$$

Here we use the notation $f^{u}:=f_{1}^{u_{1}} \ldots f_{r}^{u_{r}}$ for $u \in E=\mathbb{Z}^{r}$. Note that the open affine subsets $\bar{X}_{\gamma_{0}} \subset \bar{X}$ do not depend on the particular choices of the lattice vectors $u \in \gamma_{0}^{\circ}$. The basic observation is the following.

Proposition 2.9. There is a good quotient $\widehat{X} \rightarrow \widehat{X} / / T$ for the $T$-action on $\widehat{X}$.

Proof. First consider any two cones $\gamma_{i}, \gamma_{j} \in \operatorname{cov}(\Phi)$. Since $Q\left(\gamma_{i}\right)^{\circ} \cap Q\left(\gamma_{j}\right)^{\circ}$ is nonempty, we find $u^{i} \in \gamma_{i}^{\circ}$ and $u^{j} \in \gamma_{j}^{\circ}$ with $Q\left(u^{i}\right)=Q\left(u^{j}\right)$. Let $f_{i}, f_{j} \in R$ denote the 
functions corresponding to $u^{i}, u^{j}$. Then we obtain a commutative diagram

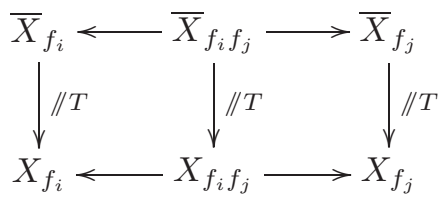

where the upper horizontal maps are open embeddings, the downwards maps are good quotients for the respective $T$-actions, and the lower horizontal arrows indicate the induced morphisms of the affine quotient spaces.

By the choice of $f_{i}$ and $f_{j}$, the quotient $f_{j} / f_{i}$ is an invariant function on $\bar{X}_{f_{i}}$, and the inclusion $\bar{X}_{f_{i} f_{j}} \subset \bar{X}_{f_{i}}$ is just the localization by $f_{j} / f_{i}$. Since $f_{j} / f_{i}$ is invariant, the latter holds as well for the quotient spaces; that means that the map $X_{f_{i} f_{j}} \rightarrow X_{f_{i}}$ is localization by $f_{j} / f_{i}$.

In particular, $X_{f_{i} f_{j}} \rightarrow X_{f_{i}}$ are open embeddings, and we may glue the maps $\bar{X}_{f_{i}} \rightarrow X_{f_{i}}$ along $\bar{X}_{f_{i} f_{j}} \rightarrow X_{f_{i} f_{j}}$. This gives a good quotient $\widehat{X} \rightarrow \widehat{X} / / T$ for the $T$-action. Note that the quotient space is separated, because the multiplication maps $\mathcal{O}\left(X_{f_{i}}\right) \otimes \mathcal{O}\left(X_{f_{j}}\right) \rightarrow \mathcal{O}\left(X_{f_{i} f_{j}}\right)$ are surjective.

Definition 2.10. In the notation of Proposition 2.9 the variety associated to the bunched ring $(R, \mathfrak{F}, \Phi)$ is the quotient space $X(R, \mathfrak{F}, \Phi):=\widehat{X} / / T$.

For the subsequent studies and results, it is important to observe that the variety $X:=X(R, \mathfrak{F}, \Phi)$ associated to a bunched $\operatorname{ring}(R, \mathfrak{F}, \Phi)$ inherits a certain decomposition from its construction.

Consider the quotient map $p_{X}: \widehat{X} \rightarrow X$. Then, for any $\gamma_{0} \in \operatorname{rlv}(\Phi)$, we have the open affine subset $X_{\gamma_{0}}:=\bar{X}_{\gamma_{0}} / / T$ of $X=\widehat{X} / / T$. Note that $p_{X}^{-1}\left(X_{\gamma_{0}}\right)=\bar{X}_{\gamma_{0}}$ holds, and for a further $\gamma_{1} \in \operatorname{rlv}(\Phi)$ with $\gamma_{0} \preceq \gamma_{1}$, we have $X_{\gamma_{1}} \subset X_{\gamma_{0}}$ and $\bar{X}_{\gamma_{1}} \subset \bar{X}_{\gamma_{0}}$. We define locally closed subsets in $X$ and $\widehat{X}$ :

$$
X\left(\gamma_{0}\right):=X_{\gamma_{0}} \backslash \bigcup_{\gamma_{0} \prec \gamma_{1} \in \operatorname{rlv}(\Phi)} X_{\gamma_{1}}, \quad \widehat{X}\left(\gamma_{0}\right):=p_{X}^{-1}\left(X\left(\gamma_{0}\right)\right) .
$$

Proposition 2.11. The variety $X:=X(R, \mathfrak{F}, \Phi)$ associated to the bunched ring $(R, \mathfrak{F}, \Phi)$ is a disjoint union of non-empty locally closed subsets:

$$
X=\bigcup_{\gamma_{0} \in \operatorname{rlv}(\Phi)} X\left(\gamma_{0}\right) .
$$

Moreover, the strata $\widehat{X}\left(\gamma_{0}\right), \gamma_{0} \in \operatorname{rlv}(\Phi)$, of the lifted decomposition on $\widehat{X}$ are explicitly given by

$$
\widehat{X}\left(\gamma_{0}\right)=\left\{x \in \bar{X} ; \prod_{e_{i} \in \gamma_{0}} f_{i}(x) \neq 0, \forall \gamma_{0} \prec \gamma_{1} \in \operatorname{rlv}(\Phi): \prod_{e_{j} \in \gamma_{1}} f_{j}(x)=0\right\} .
$$

In order to illustrate the construction of the variety associated to a bunched ring, we look again at Examples 2.4, 2.5 and 2.6.

Example 2.12. Let $(R, \mathfrak{F}, \Theta)$ be a trivially bunched ring as in Example 2.4. Then the associated variety is $X(R, \mathfrak{F}, \Theta)=\operatorname{Spec}(R)$.

Example 2.13. Let $(R, \mathfrak{F}, \Theta)$ be a bunched polynomial ring as in Example 2.5, Then $X(R, \mathfrak{F}, \Theta)$ is the toric variety corresponding to $\Theta$, and the decomposition of $X(R, \mathfrak{F}, \Theta)$ is the orbit stratification. 
Example 2.14. Consider the bunched ring $(R, \mathfrak{F}, \Phi)$ of Example 2.6. Then the associated variety $X(R, \mathfrak{F}, \Phi)$ equals the Grassmannian $G(2,4)$.

Indeed, $\bar{X}:=\operatorname{Spec}(R)$ is the affine cone over $G(2,4)$ (see, e.g., [30, Sec. 5.13]), and the action of $T:=\operatorname{Spec}(\mathbb{K}[K])$ on $\bar{X}$ is the usual $\mathbb{C}^{*}$-action. Moreover, $\widehat{X}=\bar{X} \backslash\{0\}$, because $\operatorname{cov}(\Phi)$ consists of all rays of $\gamma$, and thus $\widehat{X}$ consists of all $x \in \bar{X}$ admitting an $f_{j}$ with $f_{j}(x) \neq 0$. Thus, $X(R, \mathfrak{F}, \Phi)=\widehat{X} / / T$ equals $G(2,4)$.

\section{TORIC AMBIENT VARIETIES}

In the previous section, we presented the basic construction of the paper, which associates to any bunched ring a stratified variety. In this section we show that these varieties admit closed embeddings into toric varieties such that the stratification is induced from the orbit stratification.

Throughout the whole section, we fix a bunched ring $(R, \mathfrak{F}, \Phi)$, and we denote the projected cone associated to $\mathfrak{F}$ by $(E \stackrel{Q}{\longrightarrow} K, \gamma)$. The construction of toric ambient varieties is based on the following observation.

Lemma 3.1. There exists a bunch $\Theta$ in $(E \stackrel{Q}{\longrightarrow} K, \gamma)$ such that $\sigma^{\circ} \cap \tau^{\circ} \neq \emptyset$ holds for any $\sigma \in \Theta$ and any $\tau \in \Phi$. Moreover, for every such bunch $\Theta$ one has

$$
\operatorname{rlv}(\Phi)=\left\{\gamma_{0} \in \operatorname{rlv}(\Theta) ; \gamma_{0} \text { is an } \mathfrak{F} \text {-face }\right\} .
$$

Note that for $\Theta$ and $\Phi$ as in this lemma, every cone of $\Phi$ contains in its relative interior the relative interior of some cone of $\Theta$.

Proof of Lemma 3.1. To verify the first statement, we construct $\Theta$ as follows: let $\Theta_{0}:=\Phi$. If there is a projected face $\tau \notin \Theta_{0}$ such that $\tau^{\circ} \cap \sigma^{\circ} \neq \emptyset$ holds for all $\sigma \in \Theta_{0}$, then we set $\Theta_{1}:=\Theta_{0} \cup\{\tau\}$. After finitely many such steps, we arrive at a collection $\Theta_{m}$, which can no longer be enlarged in the above sense. Then the set $\Theta$ of minimal cones of $\Theta_{m}$ is a bunch, as desired.

We turn to the second statement. Let $\gamma_{0} \in \operatorname{rlv}(\Phi)$. Then $\gamma_{0}$ is an $\mathfrak{F}$-face. Moreover, $Q\left(\gamma_{0}\right)^{\circ} \supset \tau^{\circ}$ holds for some $\tau \in \Phi$. Hence $Q\left(\gamma_{0}\right)^{\circ}$ intersects all $\sigma^{\circ}$, where $\sigma \in \Theta$. By the defining property 1.1(i) of a bunch, $Q\left(\gamma_{0}\right)^{\circ} \supset \sigma^{\circ}$ holds for some $\sigma \in \Theta$. This in turn implies $\gamma_{0} \in \operatorname{rlv}(\Theta)$.

Conversely, let $\gamma_{0} \in \operatorname{rlv}(\Theta)$ be an $\mathfrak{F}$-face. Then there is a $\sigma \in \Theta$ such that $Q\left(\gamma_{0}\right)^{\circ}$ contains $\sigma^{\circ}$. Thus the open projected $\mathfrak{F}$-face $Q\left(\gamma_{0}\right)^{\circ}$ meets $\tau^{\circ}$ for every $\tau \in \Phi$. By the properties 2.1(ii) of an $\mathfrak{F}$-bunch, we have $Q\left(\gamma_{0}\right)^{\circ} \supset \tau^{\circ}$ for some $\tau \in \Phi$. But this means $\gamma_{0} \in \operatorname{rlv}(\Phi)$.

Now, fix any bunch $\Theta$ in $(E \stackrel{Q}{\longrightarrow} K, \gamma)$ as provided by Lemma 3.1. Let $e_{1}, \ldots, e_{r}$ denote the canonical base vectors of $E=\mathbb{Z}^{r}$. The semigroup algebra $\mathbb{K}[\gamma \cap E]$ becomes $K$-graded by defining the degree of $\chi^{e_{i}}$ to be $Q\left(e_{i}\right)$. Moreover, we have a $K$-graded surjection

$$
\mathbb{K}[\gamma \cap E] \rightarrow R, \quad \chi^{e_{i}} \mapsto f_{i} .
$$

Geometrically, this corresponds to a closed embedding of $\bar{X}:=\operatorname{Spec}(R)$ into the toric variety $\bar{Z}:=\operatorname{Spec}(\mathbb{K}[\gamma \cap E])=\mathbb{K}^{r}$, and this embedding is equivariant with respect to the actions of the torus $T:=\operatorname{Spec}(\mathbb{K}[K])$ on $\bar{X}$ and $\bar{Z}$ defined by the $K$-gradings of $R$ and $\mathbb{K}[\gamma \cap E]$.

Let $\widehat{\Delta}$ be the maximal projectable fan associated to the bunch $\Theta$, and let $\widehat{Z} \subset \bar{Z}$ be the corresponding open toric subvariety. Let $Z$ be the toric variety arising from the quotient fan $\Delta$ of $\widehat{\Delta}$, i.e., there is a good quotient $p_{Z}: \widehat{Z} \rightarrow Z$. For $\gamma_{0} \in \operatorname{rlv}(\Theta)$, 
let $z\left(\gamma_{0}\right) \in Z$ denote the corresponding distinguished point. This means that the orbit stratification reads as

$$
Z=\bigcup_{\gamma_{0} \in \operatorname{rlv}(\Theta)} T_{Z} \cdot z\left(\gamma_{0}\right)
$$

Now, recall from the preceding section that $X:=X(R, \mathfrak{F}, \Theta)$ was defined as the good quotient of an invariant open subset $\widehat{X} \subset \bar{X}$ by the $T$-action. The meaning of the toric varieties $\widehat{Z}$ and $Z$ is the following.

Proposition 3.2. We have $\widehat{X}=\bar{X} \cap \widehat{Z}$, and there is a commutative diagram, where the horizontal maps are closed embeddings:

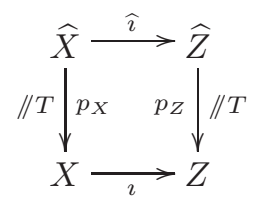

Moreover, the decompositions of $X$ and $Z$ are compatible: a cone $\gamma_{0} \in \operatorname{rlv}(\Theta)$ satisfies $X \cap T_{Z} \cdot z\left(\gamma_{0}\right) \neq \emptyset$ if and only if $\gamma_{0}$ is an $\mathfrak{F}$-face; and in that case one has

$$
X\left(\gamma_{0}\right)=X \cap T_{Z} \cdot z\left(\gamma_{0}\right) .
$$

Note that a toric variety as in this proposition is not determined by its properties. Nevertheless, the embedding construction will play an important role, and hence we define:

Definition 3.3. Let $(R, \mathfrak{F}, \Phi)$ be a bunched ring, and let $\Theta$ be a bunch as in Lemma 3.1. Then we say that $\Theta$ extends $\Phi$, and that the toric variety $Z$ defined by $\Theta$ is an ambient toric variety of $X(R, \mathfrak{F}, \Phi)$.

Proof of Proposition 3.2. Consider the decomposition of the toric variety $\widehat{Z}$ obtained by lifting the orbit stratification of $Z$ with respect to the quotient map $p_{Z}: \widehat{Z} \rightarrow Z:$

$$
\widehat{Z}=\bigcup_{\gamma_{0} \in \operatorname{rlv}(\Theta)} \widehat{Z}\left(\gamma_{0}\right), \quad \text { where } \widehat{Z}\left(\gamma_{0}\right):=p_{Z}^{-1}\left(T_{Z} \cdot z\left(\gamma_{0}\right)\right) .
$$

One can express this lifted stratification explicitly as well, in terms of the coordinates $\chi^{e_{i}}=z_{i}$ of $\bar{Z}=\mathbb{K}^{r}$. Namely, one directly verifies

$$
\widehat{Z}\left(\gamma_{0}\right)=\left\{z \in \bar{Z} ; \prod_{e_{i} \in \gamma_{0}} z_{i} \neq 0, \forall \gamma_{0} \prec \gamma_{1} \in \operatorname{rlv}(\Theta): \prod_{e_{j} \in \gamma_{1}} z_{j}=0\right\} .
$$

In view of the description of the decomposition of $X$ given in Proposition 2.11, we only have to verify the following claim: for $\gamma_{0} \in \operatorname{rlv}(\Theta)$, one has $\bar{X} \cap \widehat{Z}\left(\gamma_{0}\right) \neq \emptyset$ if and only if $\gamma_{0}$ is an $\mathfrak{F}$-face. One implication is easy: if $\gamma_{0}$ is an $\mathfrak{F}$-face, then the $T_{\widehat{Z}}$-orbit of the distinguished point $\widehat{Z}\left(\gamma_{0}\right) \in \widehat{Z}$ corresponding to $\gamma_{0}^{*}$ lies in $\widehat{Z}\left(\gamma_{0}\right)$, and it contains a point of $\bar{X} \cap \widehat{Z}\left(\gamma_{0}\right)$.

For the converse, note first that $T_{\widehat{Z}} \cdot \widehat{z}\left(\gamma_{0}\right)$ is closed in $\widehat{Z}\left(\gamma_{0}\right)$ and that it maps onto $T_{Z} \cdot z\left(\gamma_{0}\right)$. Now, consider a point $x \in \bar{X} \cap \widehat{Z}\left(\gamma_{0}\right)$. Then $p_{Z}(x)$ belongs to $T_{Z} \cdot z\left(\gamma_{0}\right)$. Hence, by basic properties of good quotients, the closures of $T \cdot x$ and $T_{\widehat{Z}} \cdot \widehat{z}\left(\gamma_{0}\right)$ have a point in common, say $x^{\prime}$. By $T$-invariance, $x^{\prime} \in \bar{X}$ holds, and by construction, we have $f_{i}\left(x^{\prime}\right) \neq 0$ if and only if $e_{i} \in \gamma_{0}$. 
Example 3.4. Consider the bunched ring $(R, \mathfrak{F}, \Phi)$ of Example 2.6 Then $\Theta:=$ $\left\{\mathbb{Q}_{\geq 0}\right\}$ extends $\Phi$, the associated toric variety is $\mathbb{P}_{5}$, and there is a diagram

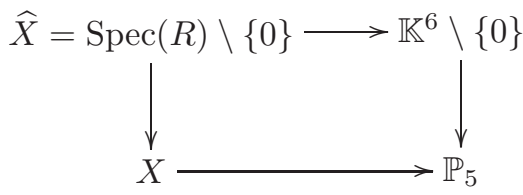

Note that the resulting embedding of $X$ is just the Plücker Embedding of the Grassmannian $G(2,4)=X$ into the projective space $\mathbb{P}_{5}$.

We now present an intrinsic characterization of the $\mathfrak{F}$-bunch $\Phi$ and the stratification of $X$. For this, we first observe that "most" of the variety $X$ is contained in the "big" orbits of the ambient toric variety $Z$. Denote by $W_{Z} \subset Z$ and $W_{\bar{Z}} \subset \bar{Z}$ the unions of all at most one-codimensional orbits of the respective big tori.

Lemma 3.5. Set $W_{X}:=X \cap W_{Z}$ and $W_{\bar{X}}:=\bar{X} \cap W_{\bar{Z}}$. Then the respective complements of these sets in $X$ and $\bar{X}$ are of codimension at least two. Moreover, we have $W_{\bar{X}}=p_{X}^{-1}\left(W_{X}\right)$, and $T$ acts freely on this set.

Proof. First recall from Proposition 1.5 that the corresponding statements hold for the sets $W_{Z}$ and $W_{\bar{Z}}$. Thus, $T$ acts freely on $W_{\bar{X}}$, and the commutative diagram of Proposition 3.2 shows $W_{\bar{X}}=p_{X}^{-1}\left(W_{X}\right)$.

It remains to verify the statements on the codimensions. For this, we describe $W_{\bar{Z}}$ in terms of the coordinates $\chi^{e_{i}}=z_{i}$ of $\bar{Z}=\mathbb{K}^{r}$. It is given by

$$
W_{\bar{Z}}=\bar{Z} \backslash \bigcup_{i<j} V\left(\bar{Z} ; z_{i}, z_{j}\right)
$$

According to the fact that $f_{i}=\left.z_{i}\right|_{\bar{X}}$ holds, we obtain

$$
W_{\bar{X}}=\bar{X} \backslash \bigcup_{i<j} V\left(\bar{X} ; f_{i}, f_{j}\right) \text {. }
$$

Since the functions $f_{i}$ are pairwise coprime, we can conclude that the complement $\bar{X} \backslash p_{X}^{-1}\left(W_{X}\right)$ is of codimension at least two in $\bar{X}$. Using semicontinuity of the fibre dimension, one obtains the analogous statement for $X \backslash W_{X}$ in $X$.

This lemma allows us to define a pullback $\imath^{*}: \operatorname{WDiv}^{T z}(Z) \rightarrow \operatorname{WDiv}(X)$ for invariant Weil divisors (note that by Proposition 3.2, $X$ meets the big torus of $Z$ ): first restrict to the smooth set $W_{Z} \subset Z$, then pull back Cartier divisors to $W_{X}$, and, finally, apply the unique extension $\operatorname{CDiv}\left(W_{X}\right) \rightarrow \mathrm{WDiv}(X)$.

Similarly, we can define a pullback of Weil divisors $p_{X}^{*}: \operatorname{WDiv}(X) \rightarrow \operatorname{WDiv}(\widehat{X})$. As a quotient space of the locally factorial variety $W_{\bar{X}}$ by a free torus action, $W_{X}$ is again locally factorial. Hence we have a well-defined pullback $W \operatorname{Div}\left(W_{X}\right) \rightarrow$ $\operatorname{WDiv}\left(W_{\bar{X}}\right)$. Again, the observation on the codimensions of the complements gives the desired extension.

Proposition 3.6. Let $D_{Z}^{i}$ be the invariant prime divisors of the ambient toric variety $Z$, numbered in such a way that $p_{Z}^{*}\left(D_{Z}^{i}\right)=\operatorname{div}\left(\chi^{e_{i}}\right)$, and set $D_{X}^{i}:=\imath^{*}\left(D_{Z}^{i}\right)$.

(i) The pullbacks with respect to $p_{X}: \widehat{X} \rightarrow X$ satisfy $p_{X}^{*}\left(D_{X}^{i}\right)=\operatorname{div}\left(f_{i}\right)$. 
(ii) A face $\gamma_{0} \preceq \gamma$ belongs to $\operatorname{rlv}(\Phi)$ if and only if there is a point $x \in X$ with

$$
x \in \bigcap_{e_{i} \notin \gamma_{0}} D_{X}^{i} \backslash \bigcup_{e_{i} \in \gamma_{0}} D_{X}^{i} .
$$

(iii) For each $\gamma \in \operatorname{rlv}(\Phi)$, the stratum $X\left(\gamma_{0}\right) \subset X$ consists precisely of the points $x \in X$ satisfying the condition of (ii).

Proof. The first statement follows immediately from functoriality of the pullback and from

$$
\operatorname{div}\left(f_{i}\right)=\widehat{\imath}^{*}\left(\operatorname{div}\left(\chi^{e_{i}}\right)\right), \quad \operatorname{div}\left(\chi^{e_{i}}\right)=p_{Z}^{*}\left(D_{Z}^{i}\right) .
$$

The second and the third statement follow from Proposition 3.2 and the corresponding description of the toric orbit stratification in terms of the $D_{Z}^{i}$; see, e.g., [8].

As mentioned, the ambient toric varieties need not be unique. One may overcome this problem by admitting ambient toric varieties that do not come from maximal projectable fans. Consider the following collection of cones in the lattice $N:=$ $\operatorname{ker}(Q)^{\vee}$ :

$$
\Delta(R, \mathfrak{F}, \Phi):=\left\{\sigma \subset N_{\mathbb{Q}} ; \sigma \preceq P\left(\gamma_{0}^{*}\right) \text { for some } \gamma_{0} \in \operatorname{rlv}(\Phi)\right\} .
$$

This is a fan. Let $Z(R, \mathfrak{F}, \Phi)$ denote the corresponding open toric variety. Then, by construction, we have:

Proposition 3.7. $Z(R, \mathfrak{F}, \Phi)$ is an open toric subvariety of any ambient toric variety of $X=X(R, \mathfrak{F}, \Phi)$, it contains $X$ as a closed subvariety, and every closed orbit of the big torus of $Z(R, \mathfrak{F}, \Phi)$ meets $X$.

\section{Geometric characterization}

In this section we characterize the class of varieties arising from bunched rings in terms of their geometric properties. As we will show in Theorem 4.2, among the varieties with finitely generated total coordinate ring, one obtains precisely those that are in a certain sense maximal with the property of being embeddable into a toric variety.

We first recall the precise definitions. Following Włodarczyk [31, we say that $X$ is an $A_{2}$-variety if any two points $x, x^{\prime} \in X$ admit a common affine neighbourhood in $X$. According to [31, Theorem A], a normal variety is $A_{2}$ if and only if it admits a closed embedding into a toric variety.

Definition 4.1. We say that an $A_{2}$-variety $X$ is $A_{2}$-maximal if it does not admit an open embedding $X \subsetneq X^{\prime}$ into an $A_{2}$-variety $X^{\prime}$ such that $X^{\prime} \backslash X$ is of codimension at least two in $X^{\prime}$.

Next, we recall the total coordinate ring studied in 9 : let $X$ be a normal variety with $\mathcal{O}^{*}(X)=\mathbb{K}^{*}$ and finitely generated free divisor class group $\mathrm{Cl}(X)$. Choose a subgroup $K \subset \mathrm{WDiv}(X)$ of the group of Weil divisors such that the canonical map $K \rightarrow \mathrm{Cl}(X)$ is an isomorphism. Then this gives a total coordinate ring (depending only up to isomorphism on the choice made):

$$
\mathcal{R}(X):=\bigoplus_{D \in K} \Gamma(X, \mathcal{O}(D)) .
$$


Theorem 4.2. Let $(R, \mathfrak{F}, \Phi)$ be a bunched ring, graded by the lattice $K$. Then the associated $X:=X(R, \mathfrak{F}, \Phi)$ is a normal $A_{2}$-maximal variety, and it satisfies

$\mathcal{O}^{*}(X)=\mathbb{K}^{*}, \quad \mathrm{Cl}(X) \cong K, \quad \mathcal{R}(X) \cong R, \quad \operatorname{dim}(X)=\operatorname{dim}(R)-\operatorname{rank}(K)$.

Conversely, every normal $A_{2}$-maximal variety $X$ with $\mathcal{O}^{*}(X)=\mathbb{K}^{*}$, finitely generated free divisor class group $\mathrm{Cl}(X)$, and finitely generated total coordinate ring $\mathcal{R}(X)$ arises from a bunched ring.

An important ingredient of the proof is factoriality of the total coordinate ring. This holds in fact for any noetherian scheme with a finitely generated divisor class group; see [9, Corollary 1.2]. In our situation, it can also be deduced from results of [3].

Proposition 4.3. Let $X$ be a normal variety with $\mathcal{O}^{*}(X)=\mathbb{K}^{*}$ and finitely generated free divisor class group. Then the following hold:

(i) The total coordinate ring $\mathcal{R}(X)$ of $X$ is a factorial ring satisfying $\mathcal{R}(X)^{*}=$ $\mathbb{K}^{*}$, and its grading is faithful.

(ii) If $\mathcal{R}(X)$ is finitely generated, then it admits a system of homogeneous pairwise non-associated non-zero prime generators.

Proof. The open set $X^{\prime} \subset X$ of smooth points of $X$ satisfies $\operatorname{Pic}\left(X^{\prime}\right)=\mathrm{Cl}(X)$, and has homogeneous coordinate ring $\mathcal{A}\left(X^{\prime}\right)=\mathcal{R}(X)$ in the sense of [3]. Thus, 3 , Remark 8.8 and Prop. 8.4] yield that $\mathcal{R}(X)$ is factorial. Moreover, $\mathcal{R}(X)$ is faithfully graded, because any divisor class is represented by a difference of two effective divisors.

In order to see $\mathcal{R}(X)^{*}=\mathbb{K}^{*}$, consider an element $f \in \mathcal{R}(X)^{*}$ and its inverse $g \in \mathcal{R}(X)^{*}$. Since $f g=1$ is homogeneous, so $f$ and $g$ are also, having Weil divisors $D$ and $E$ as their respective degrees. We obtain $D+E=0$, and considering the zero set of $f g$ gives

$$
\emptyset=Z(f g)=\operatorname{Supp}(\operatorname{div}(f g)+D+E)=\operatorname{Supp}(\operatorname{div}(f)+D) \cup \operatorname{Supp}(\operatorname{div}(g)+E) .
$$

This implies $D=-\operatorname{div}(f)$ and $E=-\operatorname{div}(g)$. In other words, $D$ and $E$ are principal. Since the grading group of $\mathcal{R}(X)$ maps isomorphically onto $\mathrm{Cl}(X)$, we can conclude $D=E=0$. Thus, we obtain $f, g \in \mathcal{O}^{*}(X)=\mathbb{K}^{*}$, and the first assertion is proved.

As to the second assertion, let $\left\{g_{1}, \ldots, g_{s}\right\}$ be any system of non-zero homogeneous generators of $\mathcal{R}(X)$. Since $\mathcal{R}^{*}(X)=\mathbb{K}^{*}$ holds, we may assume that no $g_{i}$ is a unit in $\mathcal{R}(X)$. Decompose each $g_{i}$ into prime factors, and fix a system $\left\{f_{1}, \ldots, f_{r}\right\}$ of pairwise non-associated representatives for the prime factors occuring in $g_{1}, \ldots, g_{s}$. Then the $f_{j}$ are homogeneous, and they generate $\mathcal{R}(X)$ as well.

Now we turn to the proof of the theorem. It is split into two parts. In the first part, we prove in particular the second assertion of the theorem, and then, in the second part, the first assertion is verified.

Proof of Theorem 4.2, Part 1. Let $X$ be a normal $A_{2}$-variety $X$ with $\mathcal{O}^{*}(X)=\mathbb{K}^{*}$, finitely generated free class divisor group, and finitely generated total coordinate $\operatorname{ring} R:=\mathcal{R}(X)$.

Assertion 4.2 a). For any system $\mathfrak{F} \subset R$ of non-zero homogeneous pairwise nonassociated prime generators, there is an $\mathfrak{F}$-bunch $\Phi$ and an open embedding $X \subset$ $X(R, \mathfrak{F}, \Phi)$ such that the complement is of codimension at least two. 
We prove this assertion. Choose a sublattice $K \subset \mathrm{WDiv}(X)$ of the group of Weil divisors such that the canonical map $K \rightarrow \mathrm{Cl}(X)$ is an isomorphism. Consider the associated sheaf of $K$-graded $\mathcal{O}_{X}$-algebras

$$
\mathcal{R}:=\bigoplus_{D \in K} \mathcal{O}_{X}(D)
$$

According to Proposition 4.3(i), the ring $R=\mathcal{R}(X)$ of global sections is a unique factorization domain, and it satisfies $R^{*}=\mathbb{K}^{*}$. Moreover, by assumption, $R$ is finitely generated. Let $\bar{X}:=\operatorname{Spec}(R)$, and consider the relative spectrum $\widehat{X}:=\operatorname{Spec}_{X}(\mathcal{R})$ with the canonical affine map $p_{X}: \widehat{X} \rightarrow X$.

We show that the relative spectrum $\hat{X}$ is an open subvariety of $\bar{X}$. For this, note first that $\widehat{X}$ is covered by open affine varieties of the form $p_{X}^{-1}\left(X_{f}\right)$, where $f \in R$ is homogeneous and $X_{f}$ is defined as

$$
X_{f}:=X \backslash \operatorname{Supp}(\operatorname{div}(f)+D) .
$$

This is due to the fact that every affine open $U \subset X$ is of the form $U=X_{f}$, because $X \backslash U$ is the support of some effective Weil divisor, which in turn occurs as the zero set of a section $f \in \Gamma\left(X, \mathcal{O}_{D}\right) \subset R$ of some $D \in K \cong \mathrm{Cl}(X)$.

Next note that the canonical morphism $\widehat{X} \rightarrow \bar{X}$ maps $p_{X}^{-1}\left(X_{f}\right)$ isomorphically onto $\bar{X}_{f}$. This follows from the fact that, if $f \in R$ is homogeneous, say of degree $D \in K$, then we have

$$
\mathcal{R}\left(X_{f}\right)=R_{f} .
$$

In conclusion, we obtain that $\widehat{X} \rightarrow \bar{X}$ is a local isomorphism and hence an open embedding (use, e.g., Zariski's Main Theorem). In particular, we see that $\widehat{X}$ is quasiaffine and that $p_{X}^{-1}\left(X_{f}\right)$ equals $\bar{X}_{f}$.

We shall make use of the actions of the torus $T:=\operatorname{Spec}(\mathbb{K}[K])$ on $\bar{X}$ and $\widehat{X}$ defined by the $K$-grading of the $\mathcal{O}_{X}$-algebra $\mathcal{R}$. First note that the open embedding $\widehat{X} \rightarrow \bar{X}$ is $T$-equivariant, and that the canonical morphism $p_{X}: \widehat{X} \rightarrow X$ is a good quotient for the $T$-action.

Proposition 4.3(ii) enables us to fix a system $\mathfrak{F}=\left(f_{1}, \ldots, f_{r}\right)$ of pairwise nonassociated homogeneous prime elements that generate $R=\mathcal{R}(X)$. Moreover, let $T$ act diagonally on $\mathbb{K}^{r}$ such that the coordinate $z_{i}$ has the same degree as the function $f_{i}$. This gives rise to a $T$-equivariant closed embedding

$$
\bar{X} \rightarrow \mathbb{K}^{r}, \quad x \mapsto\left(f_{1}(x), \ldots, f_{r}(x)\right) .
$$

The first major step is to show that the good quotient $p_{X}: \widehat{X} \rightarrow X$ by $T$ admits a toric extension, that means that there is an open toric subvariety $\widehat{Z} \subset \mathbb{K}^{r}$ admitting a good quotient $p_{Z}: \widehat{Z} \rightarrow Z$ for the $T$-action such that $\widehat{X}=\widehat{Z} \cap \bar{X}$ holds. Note that then restricting $p_{Z}$ to $\widehat{X}$ gives back the initial map $p_{X}: \widehat{X} \rightarrow X$.

Let $\mathbb{T}^{r} \subset \mathbb{K}^{r}$ denote the standard $r$-torus. The candidate for our $\widehat{Z}$ is the minimal $\mathbb{T}^{r}$-invariant open subset of $\mathbb{K}^{r}$, which contains $\widehat{X}$ :

$$
\widehat{Z}:=\left\{z \in \mathbb{K}^{r} ; \overline{\mathbb{T}^{r} \cdot z} \cap \widehat{X} \neq \emptyset\right\} .
$$

Then $\widehat{Z}$ satisfies $\widehat{X}=\widehat{Z} \cap \bar{X}$. Indeed, the inclusion "С" is clear by definition. For the reverse inclusion, recall that $\widehat{X}$ is the union of the subsets $\bar{X}_{f_{i}}$, and that 
$f_{i}=\left.z_{i}\right|_{\bar{X}}$ holds. Thus, we have

$$
\widehat{Z} \cap \bar{X} \subset \bigcup_{i=1}^{r} \mathbb{K}_{z_{i}}^{r} \cap \bar{X}=\widehat{X} .
$$

Our task is to show that $\widehat{Z}$ admits a good quotient. To verify this, it suffices to show that any two points $z, z^{\prime} \in \widehat{Z}$ admit a common $T$-invariant affine neighbourhood in $\widehat{Z}$; see $[6$, Theorem $\mathrm{C}]$. The latter property will be established by refining an argument used in [14. Choose

$$
x \in \overline{\mathbb{T}^{r} \cdot z} \cap \bar{X}, \quad x^{\prime} \in \overline{\mathbb{T}^{r} \cdot z^{\prime}} \cap \bar{X} .
$$

Then it is enough to show that there is a common $T$-invariant affine open neighbourhood $U \subset \widehat{Z}$ of $x$ and $x^{\prime}$. Namely, any such $U$ intersects the $\mathbb{T}^{r}$-orbits of $z$ and $z^{\prime}$. Thus, as an intersection of non-empty open subsets of $\mathbb{T}^{r}$, the set

$$
V:=\left\{t \in \mathbb{T}^{r} ; t^{-1} \cdot z \in U\right\} \cap\left\{t \in \mathbb{T}^{r} ; t^{-1} \cdot z^{\prime} \in U\right\}
$$

is non-empty. Take any element $t_{0} \in V$. Then the translate $t_{0} \cdot U$ contains both points, $z$ and $z^{\prime}$, and hence it is the desired common $T$-invariant affine open neighbourhood of $z$ and $z^{\prime}$ in $\widehat{Z}$.

In order to show the existence of a common $T$-invariant neighbourhood $U$ for the points $x$ and $x^{\prime}$, we make use of the $A_{2}$-property of $X$ : we choose a common affine open neighbourhood $X_{0} \subset X$ of the images $p_{X}(x)$ and $p_{X}\left(x^{\prime}\right)$.

Then the inverse image $p_{X}^{-1}\left(X_{0}\right)$ is affine, and hence its complement in $\bar{X}$ is the support of an effective Weil divisor. Thus, by factoriality of $R$, there is a $T$ homogeneous $h \in R$ with $p_{X}^{-1}\left(X_{0}\right)=\bar{X}_{h}$. As the $f_{i}$ generate $R$, we can write $h=H\left(f_{1}, \ldots, f_{r}\right)$ for some $T$-homogeneous polynomial $H \in \mathbb{K}\left[z_{1}, \ldots, z_{r}\right]$.

Consider the good quotient $q: \mathbb{K}_{H}^{r} \rightarrow \mathbb{K}_{H}^{r} / / T$, which exists because $\mathbb{K}_{H}^{r}$ is an affine variety. Then we have

$$
q\left(\overline{T \cdot x} \cup \overline{T \cdot x^{\prime}}\right) \cap q\left(\mathbb{K}_{H}^{r} \backslash \widehat{Z}\right)=\emptyset .
$$

Take any affine neighbourhood $V \subset \mathbb{K}_{H}^{r} / / T$ of $q(x)$ and $q\left(x^{\prime}\right)$ that does not intersect $q\left(\mathbb{K}_{H}^{r} \backslash \widehat{Z}\right)$. Then the inverse image $U:=q^{-1}(V)$ is a $T$-invariant affine neighbourhood of $x$ and $x^{\prime}$ in $\widehat{Z}$.

So, we obtained the desired toric extension $p_{Z}: \widehat{Z} \rightarrow Z$ of the good quotient $p_{X}: \widehat{X} \rightarrow X$. Now, consider any enlargement of our toric extension. This means a toric open subset $\widehat{Z}^{\prime} \subset \mathbb{K}^{r}$ admitting a good quotient $p_{Z^{\prime}}: \widehat{Z}^{\prime} \rightarrow Z^{\prime}$ for the $T$-action such that $\widehat{Z}$ is a $p_{Z^{\prime}}$-saturated subset of $\widehat{Z}^{\prime}$.

Cutting down to $\bar{X}$, we obtain an open inclusion $\widehat{X} \subset \widehat{X}^{\prime}$, and hence $X \subset X^{\prime}$ on the level of quotients. Since $\widehat{X}$ and $\widehat{X}^{\prime}$ have the same global functions, the complement $\widehat{X}^{\prime} \backslash \widehat{X}$ is of codimension at least two. By semicontinuity of the fibre dimension, the same holds for $X^{\prime} \backslash X$.

In the above procedure, we may assume that $\widehat{Z}^{\prime}$ is $T$-maximal, i.e., cannot be enlarged itself. In this situation, we shall construct an $\mathfrak{F}$-bunch $\Phi$ such that $X^{\prime}=$ $X(R, \mathfrak{F}, \Phi)$ holds. For the sake of readable notation, we perform a reset: we replace $X^{\prime}$ with $X$ and $Z^{\prime}$ with $Z$, etc.

The crucial point is to show that the toric morphism $p_{Z}: \widehat{Z} \rightarrow Z$ is a Cox construction for $Z$. For this, it is most convenient to verify the conditions of geometric characterization of Proposition [1.5. The first property is clear by construction. Thus, we are left with checking condition 1.5(ii). 
Let $V_{X} \subset X$ denote the set of smooth points, and consider the inverse image $V_{\widehat{X}}=p_{X}^{-1}\left(V_{X}\right)$. This set has a complement of codimension at least two in $\bar{X}$, because, by normality, the same holds for the subset $V_{X} \subset X$, and hence we obtain for the global functions

$$
\mathcal{O}\left(V_{\widehat{X}}\right)=\mathcal{R}\left(V_{X}\right)=R=\mathcal{O}(\bar{X}) .
$$

Moreover, $V_{\widehat{X}}$ meets the big torus $\mathbb{T}^{r} \subset \mathbb{K}^{r}$. It meets also every $\mathbb{T}^{r}$-orbit of codimension one: these orbits are the sets $B_{i}$ given by $z_{i}=0$ for some $i$ and $z_{j} \neq 0$ for all $j \neq i$. Thus, the intersection of such an orbit with $\bar{X}$ is given by

$$
B_{i} \cap \bar{X}=\left\{x \in \bar{X} ; f_{i}(x)=0, f_{j}(x) \neq 0 \text { for all } j \neq i\right\} .
$$

Since the $f_{k}$ are relatively coprime, these intersections are non-empty, and hence of codimension one in $\bar{X}$. Since $V_{\widehat{X}} \subset \bar{X}$ has a complement of codimension at least two, every $B_{i} \cap V_{\widehat{X}}$ is non-empty.

Eventually, we claim that $T$ acts freely on the set $V_{\widehat{X}}$. This is most easily seen in terms of homogeneous functions: on $V_{X}$ every divisor $D \in K$ is Cartier. Hence, locally on $V_{\widehat{X}}$, there exists an invertible homogeneous function in every degree $D \in K$. This implies freeness of the $T$-action on $V_{\widehat{X}}$.

In conclusion, for the set $W_{\bar{Z}} \subset \bar{Z}:=\mathbb{K}^{r}$ consisting of the at most one codimensional $\mathbb{T}^{r}$-orbits, the preceding observations imply that $T$ acts freely on $W_{\bar{Z}}$, that $W_{\bar{Z}} \subset \widehat{Z}$ holds, and that every fibre of $p_{Z}^{-1}\left(p_{Z}(z)\right)$, where $z \in W_{\bar{Z}}$, consists of a single (free) $T$-orbit. Thus we verified condition [1.5)(ii).

Knowing that $\widehat{Z}$ is $T$-maximal and that $p_{Z}: \widehat{Z} \rightarrow Z$ is a Cox construction, we may apply [4, Lemma 7.8]. It tells us that $p_{Z}: \widehat{Z} \rightarrow Z$ arises from a bunch $\Theta$ in the projected cone $\left(\mathbb{Z}^{r} \stackrel{Q}{\longrightarrow} K, \mathbb{Q}_{\geq 0}^{r}\right)$, where $Q$ maps each $e_{i}$ to the degree of the coordinate $z_{i}$.

Now we are ready to define the $\mathfrak{F}$-bunch $\Phi$. Consider the collection $\Omega \subset \operatorname{rlv}(\Theta)$ of all $\mathfrak{F}$-faces. Let $\Phi$ be the collection of all minimal cones among the images $Q\left(\gamma_{0}\right)$, where $\gamma_{0} \in \Omega$. Then $\Phi$ is an $\mathfrak{F}$-bunch, extended by $\Theta$. Using Proposition 3.2, it is straightforward to check that $X=X(R, \mathfrak{F}, \Phi)$ holds.

Proof of Theorem 4.2, Part 2. We verify the claimed properties for the variety $X$ arising from a bunched ring $(R, \mathfrak{F}, \Phi)$. We work in terms of $\bar{X}:=\operatorname{Spec}(R)$, the torus $T:=\operatorname{Spec}(\mathbb{K}[K])$, the subset $\widehat{X} \subset \bar{X}$ and the good quotient $p_{X}: \widehat{X} \rightarrow X$ as introduced in the construction of $X$.

First of all note that, as a good quotient space of the normal variety $\widehat{X}$, the variety $X$ is normal. Moreover, choosing any ambient toric variety $X \subset Z$, we obtain that $X$ inherits the $A_{2}$-property.

To determine the coordinate ring $\mathcal{R}(X)$, consider the subset $W_{X}=X \cap W_{Z}$ as discussed in Lemma 3.5. Then $p_{X}^{-1}\left(W_{X}\right)$ is locally factorial, and $T$ acts freely on this set. Thus, the results of $[3]$ give us the Picard group $\operatorname{Pic}\left(W_{X}\right)$ and the homogeneous coordinate ring $\mathcal{A}\left(W_{X}\right)$ as defined in [3]:

$$
\operatorname{Pic}\left(W_{X}\right) \cong K, \quad \mathcal{A}\left(W_{X}\right) \cong \mathcal{O}\left(p_{X}^{-1}\left(W_{X}\right)\right) \cong \mathcal{O}(\bar{X})=R .
$$

Since $X \backslash W_{X}$ is of codimension at least two, we obtain that $\operatorname{Pic}\left(W_{X}\right)$ is isomorphic to $\mathrm{Cl}(X)$, and that $\mathcal{A}\left(W_{X}\right)$ is isomorphic to $\mathcal{R}(X)$. In fact, we shall later provide an explicit isomorphism $K \cong \mathrm{Cl}(X)$, making the present proof independent from the reference 3 . 
It remains to show that $X$ is $A_{2}$-maximal. So, assume that there is an open embedding $X \subset X^{\prime}$ with an $A_{2}$-variety $X^{\prime}$ such that the complement $X^{\prime} \backslash X$ is of codimension at least two in $X^{\prime}$. We may assume that $X^{\prime}$ is normal; otherwise, take its normalization instead. Then the inclusion $X \subset X^{\prime}$ gives rise to commutative diagrams of isomorphisms
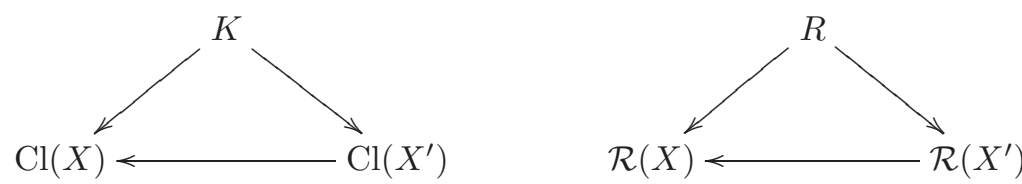

According to Proposition 4.3(ii) and Assertion 4.2 a), we find an $\mathfrak{F}$-bunch $\Phi^{\prime}$ defining a commutative diagram where the lower row is an open embedding having a complement of codimension at least two:

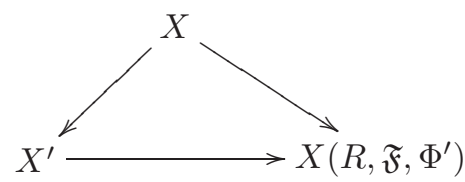

Hence, we may even assume that $X^{\prime}$ equals $X\left(R, \mathfrak{F}, \Phi^{\prime}\right)$. Now choose extending bunches $\Theta$ and $\Theta^{\prime}$ for $\Phi$ and $\Phi^{\prime}$. Consider the corresponding ambient toric varieties $Z$ and $Z^{\prime}$. Using Lemma3.5 (and its notation), we obtain the following commutative diagram:

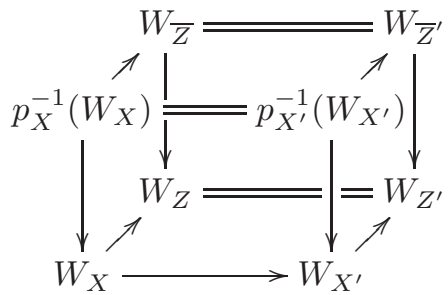

In particular, we can conclude that the induced morphism $W_{X} \rightarrow W_{X^{\prime}}$ is the identity map. Consequently, the divisors $D_{X}^{i}$ and $D_{X^{\prime}}^{i}$ considered in Proposition 3.6 satisfy

$$
\left.D_{X^{\prime}}^{i}\right|_{X}=D_{X}^{i} .
$$

Now we may use the intrinsic characterization of relevant faces provided in Proposition 3.6 (ii). It yields $\operatorname{rlv}(\Phi) \subset \operatorname{rlv}\left(\Phi^{\prime}\right)$. By the maximality properties of $\mathfrak{F}$-bunches, we obtain $\Phi=\Phi^{\prime}$. This eventually gives $X=X^{\prime}$.

We conclude this section with two immediate applications concerning toric varieties. For $\mathbb{Q}$-factorial projective varieties $X$, the first statement was also observed in [17, Cor. 2.10].

Corollary 4.4. Let $X$ be a normal, $A_{2}$-maximal variety with $\mathcal{O}(X)=\mathbb{K}$, finitely generated free divisor class group and finitely generated total coordinate ring $\mathcal{R}(X)$. Then the following statements are equivalent:

(i) $X$ is a toric variety.

(ii) $\mathcal{R}(X)$ is a polynomial ring. 
Proof. By [8], the total coordinate ring of a toric variety is a polynomial ring. Conversely, let us assume that the total coordinate ring $R:=\mathcal{R}(X)$ of $X$ is a polynomial ring. Then the set $\mathfrak{F}=\left\{T_{1}, \ldots, T_{r}\right\}$ consisting of the indeterminates is a system of generators for $R$.

Since we assumed $\mathcal{O}(X)=\mathbb{K}$, we have $R_{0}=\mathbb{K}$, and thus the torus action on $\mathbb{K}^{r}=\operatorname{Spec}(R)$ induced by the $\mathrm{Cl}(X)$-grading is linearizable; see [18. In other words, we may assume that the $T_{i}$ are homogeneous. Assertion 4.2 a) then says that $X$ arises from a bunched polynomial $\operatorname{ring}(R, \mathfrak{F}, \Phi)$, and hence is a toric variety.

Note that $A_{2}$-maximality is essential in this statement: the projective plane with four different points removed has $\mathbb{K}\left[T_{1}, T_{2}, T_{3}\right]$ as its total coordinate ring, but it can never be made into a toric variety.

Here comes a characterization of $A_{2}$-maximality for a toric variety in terms of its fan (inside the category of toric varieties, the statement is obvious).

Corollary 4.5. Let $\Delta$ be a fan in a lattice $N$, such that $N$ is generated as a lattice by the primitive vectors of the rays of $\Delta$, and $\Delta$ cannot be enlarged without adding new rays. Then the corresponding toric variety $X$ is $A_{2}$-maximal.

Proof. The assumptions on the fan $\Delta$ guarantee that $X$ arises from a bunched (polynomial) ring; compare [4, Theorem 7.3 (ii)]. Hence, Theorem 4.2 applies.

\section{Divisor ClASSES AND SINGULARITIES}

In this section, we take a closer look at the divisors of the variety $X$ associated to a given bunched $\operatorname{ring}(R, \mathfrak{F}, \Phi)$. We provide an explicit isomorphism $K \rightarrow \mathrm{Cl}(X)$ from the grading lattice onto the divisor class group, and we investigate the behaviour of divisors in terms of this isomorphism and the stratification of $X$. As an application, we study the singularities of $X$.

For the definition of the isomorphism $K \rightarrow \mathrm{Cl}(X)$, recall that $X$ is the good quotient of an open subset $\widehat{X}$ of $\bar{X}:=\operatorname{Spec}(R)$ by the action of $T:=\operatorname{Spec}(\mathbb{K}[K])$. Moreover, we showed in Lemma 3.5 that there is an open set $W_{X} \subset X$ such that $T$ acts freely on the inverse image $W_{\bar{X}}:=p_{X}^{-1}\left(W_{X}\right)$ under the quotient map $p_{X}: \widehat{X} \rightarrow X$, and both sets have small complement in $X$ and $\bar{X}$, respectively.

Lemma 5.1. For every $w \in K$, choose a $w$-homogeneous function $h_{w} \in \mathbb{K}(\widehat{X})^{*}$ and cover the set $W_{\bar{X}}$ by $T$-invariant open sets $W_{j}$ admitting $w$-homogeneous functions $h_{j} \in \mathcal{O}^{*}\left(W_{j}\right)$. Then there is a (well-defined) isomorphism

$$
\overline{\mathfrak{D}}_{X}: K \rightarrow \operatorname{Cl}(X), \quad w \mapsto \overline{\mathfrak{D}}_{X}(w), \text { where }\left.\overline{\mathfrak{D}}_{X}(w)\right|_{p_{X}\left(W_{j}\right)}=\left[\operatorname{div}\left(h_{w} / h_{j}\right)\right] .
$$

Moreover, in the notation of Proposition [3.6. we have $\overline{\mathfrak{D}}_{X}\left(w_{i}\right)=\left[D_{X}^{i}\right]$, where $w_{i}=\operatorname{deg}\left(f_{i}\right)$ for $f_{i} \in \mathfrak{F}$.

Proof. First note that the open subset $W_{\bar{X}} \subset \operatorname{Spec}(R)$ is locally factorial, and that $\operatorname{Pic}\left(W_{\bar{X}}\right)$ is trivial. Both statements hold, because $R$ is a factorial ring. From the first one we infer that, as a free $T$-quotient of the locally factorial $W_{\bar{X}}$, the variety $W_{X}$ is again locally factorial.

To proceed, we work in terms of $T$-linearized line bundles. Any $w \in K$ corresponds to a character $\chi^{w}: T \rightarrow \mathbb{K}^{*}$, and this character defines a linearization of the trivial bundle, namely

$$
t \cdot(x, z):=\left(t \cdot x, \chi^{w}(t) z\right)
$$


Thus, every $w \in K$ defines an element in the group $\operatorname{Pic}_{T}\left(W_{\bar{X}}\right)$ of equivariant isomorphy classes of $T$-linearized line bundles on $W_{\bar{X}}$. Since $\operatorname{Pic}\left(W_{\bar{X}}\right)=0$ and $\mathcal{O}^{*}(W)=\mathbb{K}^{*}$ hold, this assignment even defines an isomorphism (use 22, Lemma 2.2]):

$$
\alpha: K \rightarrow \operatorname{Pic}_{T}\left(W_{\bar{X}}\right) .
$$

Moreover, according to [22, Proposition 4.2] one obtains a pullback isomorphism $\operatorname{Pic}\left(W_{X}\right) \rightarrow \operatorname{Pic}_{T}\left(W_{\bar{X}}\right)$ by endowing each pullback bundle with the trivial linearization. Denoting by $\beta$ the inverse of this pullback isomorphism, we obtain an isomorphism

$$
\beta \circ \alpha: K \rightarrow \operatorname{Pic}\left(W_{X}\right) .
$$

Since the complement $X \backslash W_{X}$ is of codimension at least two in $X$, the isomorphism $\beta \circ \alpha$ induces an isomorphism $K \rightarrow \mathrm{Cl}(X)$. One directly verifies that this is the map $\overline{\mathfrak{D}}_{X}$ defined in the assertion.

In order to verify the last statement, recall from Proposition 3.6(i) that $p_{X}^{*}\left(D_{X}^{i}\right)$ equals $\operatorname{div}\left(f_{i}\right)$. Thus, locally, we have

$$
p_{X}^{*}\left(D_{X}^{i}\right)=\operatorname{div}\left(f_{i}\right)=\operatorname{div}\left(f_{i} / h_{j}\right)=p_{X}^{*}\left(\operatorname{div}\left(f_{i} / h_{j}\right)\right),
$$

where the $h_{j}$ are functions of degree $w_{i}$ as in the definition of $\overline{\mathfrak{D}}_{X}$. Since pullback is injective on the level of divisors, the assertion follows.

Note that the isomorphism $\overline{\mathfrak{D}}_{X}: K \rightarrow \mathrm{Cl}(X)$ does not depend on the choices of $h_{w}$ and the $h_{j}$ made in its definition.

Moreover, $\overline{\mathfrak{D}}_{X}$ is compatible with any ambient toric variety $Z$ of $X$ : in terms of the projected cone $(E \stackrel{Q}{\longrightarrow} K, \gamma)$ associated to $\mathfrak{F}$, we have a (well-defined) isomorphism (compare [4, Section 10]):

$$
\overline{\mathfrak{D}}_{Z}: K \rightarrow \operatorname{Cl}(Z), \quad w \mapsto \sum_{i=1}^{r}\left\langle\widehat{w}, e_{i}^{*}\right\rangle\left[D_{i}^{Z}\right], \quad \text { where } Q(\widehat{w})=w .
$$

Proposition 5.2. The closed embedding $\imath: X \rightarrow Z$ induces a commutative diagram of isomorphisms:

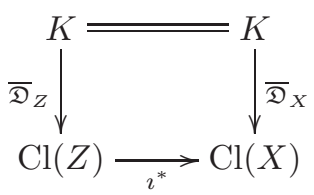

Proof. As noted earlier, Lemma 3.5 ensures that the pullback map $\imath^{*}: \mathrm{Cl}(Z) \rightarrow$ $\mathrm{Cl}(X)$ is in fact well defined. The rest is an immediate consequence of the last statement of Lemma 5.1.

In terms of the map $\overline{\mathfrak{D}}_{X}$, we can say when a given Weil divisor $D \in \operatorname{WDiv}(X)$ is $\mathbb{Q}$-Cartier or even Cartier at a point $x \in X$.

Proposition 5.3. Let $\gamma_{0} \in \operatorname{rlv}(\Phi)$, and let $x \in X\left(\gamma_{0}\right)$ be a point in the corresponding stratum.

(i) A class $\overline{\mathfrak{D}}_{X}(w)$ is $\mathbb{Q}$-Cartier at $x$ if and only if $w \in \operatorname{lin}\left(Q\left(\gamma_{0}\right)\right)$ holds.

(ii) A class $\overline{\mathfrak{D}}_{X}(w)$ is Cartier at $x$ if and only if $w \in Q\left(\operatorname{lin}\left(\gamma_{0}\right) \cap E\right)$ holds. 
Proof. It suffices to verify the second assertion. For this let $D$ represent the class $\overline{\mathfrak{D}}_{X}(w)$, and note that $D$ is Cartier at $x$ if and only if the stalk $\mathcal{O}(D)_{x}$ is generated by a single element $g_{x} \in \mathcal{O}(D)_{x}$. This is equivalent to saying that the fibre $Y:=p_{X}^{-1}(x)$ admits an invertible homogeneous element $g \in \mathcal{O}(Y)_{w}$.

Let $Z$ be any ambient toric variety of $X$, and let $\widehat{Z}$ be as in Proposition 3.2 Then the fibre $Y$ is closed in the toric variety $\widehat{Z}$, and the affine toric chart $\widehat{Z}_{\gamma_{0}^{*}} \subset$ $\widehat{Z}$ corresponding to the cone $\gamma_{0}^{*}$ is the smallest $T_{\widehat{Z}}$-invariant affine open subset containing $Y$.

Consequently, there exists an invertible element $g \in \mathcal{O}(Y)_{w}$ if and only if the toric neighbourhood $\widehat{Z}_{\gamma_{0}^{*}} \subset \widehat{Z}$ admits an invertible character function $\chi^{u} \in \mathcal{O}\left(\widehat{Z}_{\gamma_{0}^{*}}\right)_{w}$. But the latter is equivalent to the existence of a linear form $u \in \operatorname{lin}\left(\gamma_{0}\right) \cap E$ with $Q(u)=w$.

As a first application, we give basic statements on the singularities of $X=$ $X(R, \mathfrak{F}, \Phi)$. In particular, we will see that the decomposition by relevant faces is "equisingular" in a certain raw sense. We say that a point $y$ of a variety $Y$ is $\mathbb{Q}$-factorial if it is normal and, near $y$, every closed subset of codimension one is the set of zeroes of a regular function.

Corollary 5.4. Let $\gamma_{0} \in \operatorname{rlv}(\Phi)$, and consider a point $x \in X\left(\gamma_{0}\right)$.

(i) The point $x$ is factorial if and only if $Q$ maps $\operatorname{lin}\left(\gamma_{0}\right) \cap E$ onto $K$.

(ii) The point $x$ is $\mathbb{Q}$-factorial if and only if $Q\left(\gamma_{0}\right)$ is of full dimension.

Proof. This is an immediate consequence of two things: first a point $x \in X$ is factorial $(\mathbb{Q}$-factorial) if and only if every Weil divisor is Cartier $(\mathbb{Q}$-Cartier) at $x$; second, the characterization of the latter properties in terms of relevant faces performed in Proposition 5.3 .

Let us have a look at the case that the open set $\widehat{X} \subset \bar{X}$ occuring in the construction of $X$ is smooth. This situation will occur in all the examples studied later. First we note a general statement.

Proposition 5.5. Suppose that $\widehat{X}$ is smooth. Then $X$ has at most rational singularities. In particular, $X$ is Cohen-Macaulay.

Proof. This follows from general theorems saying that quotients of smooth varieties by reductive groups have at most rational singularities; see [7] and [15.

In general, there is no hope to decide in terms of combinatorial data whether or not a given $x \in X$ is a smooth point. But under the additional assumption that $\widehat{X}$ is smooth, we have the following.

Proposition 5.6. Suppose that $\widehat{X}$ is smooth, let $\gamma_{0} \in \operatorname{rlv}(\Phi)$, and let $x \in X\left(\gamma_{0}\right)$. Then $x$ is a smooth point if and only if $Q$ maps $\operatorname{lin}\left(\gamma_{0}\right) \cap E$ onto $K$.

Proof. The "only if" part is clear by Corollary 5.4 So, let $Q$ map $\operatorname{lin}\left(\gamma_{0}\right) \cap E$ onto $K$. Then the quotient presentation $p_{Z}: \widehat{Z} \rightarrow Z$ of an ambient toric variety, is a principal bundle over the affine chart $Z_{\gamma_{0}}:=p_{Z}\left(\widehat{Z}_{\gamma_{0}^{*}}\right)$ of $Z$ corresponding to $\gamma_{0}$. The latter holds as well for $\widehat{X} \rightarrow X$ over $X_{\gamma_{0}}=X \cap Z_{\gamma_{0}}$. Since we have $X\left(\gamma_{0}\right) \subset X_{\gamma_{0}}$, we see that $x$ is smooth.

According to Proposition 5.3 the behaviour of singularities is reflected in the ambient toric varieties studied in Section 3 . 
Proposition 5.7. The embedding $X \subset Z$ into an ambient toric variety $Z$ has the following property: a point $x \in X$ is a factorial $(\mathbb{Q}$-factorial) point of $X$ if and only if it is a smooth $(\mathbb{Q}$-factorial) point of $Z$.

Recall from [8], that in Cox's quotient presentation $p_{Z}: \widehat{Z} \rightarrow Z$ of a toric variety $Z$, a fibre $p_{Z}^{-1}(z)$ over a point $z \in Z$ consists of a single closed orbit if and only if $z$ is a $\mathbb{Q}$-factorial point. Thus, Corollary 5.4, and Propositions 5.7 and 3.2 fit together to produce the following statement.

Corollary 5.8. For $X=X(R, \mathfrak{F}, \Phi)$, the following statements are equivalent:

(i) $p_{X}: \widehat{X} \rightarrow X$ is a geometric quotient;

(ii) $X$ is $\mathbb{Q}$-factorial;

(iii) $\Phi$ consists of cones of full dimension.

As before, if $\widehat{X}$ is smooth, then we may replace "factorial" with "smooth" in Proposition 5.7. Moreover, if, in this setup, we take the embedding $X \subset Z(\Phi)$ discussed in Proposition 3.7. then we arrive at the statement that $X$ is smooth (Q্-factorial) if and only if $Z(\Phi)$ is smooth.

\section{Functoriality properties}

In this section, we make the assignment from bunched rings to varieties into a functor, and study basic properties of this functor. For example, we figure out when two bunched rings define isomorphic varieties; see Corollary 6.8

First, we have to introduce the concept of a morphism of bunched rings. In doing this, we follow closely the ideas of [3]. To any bunched ring $(R, \mathfrak{F}, \Phi)$ we associate an irrelevant ideal:

$$
I(R, \mathfrak{F}, \Phi):=\sqrt{\left\langle f^{u} ; u \in \gamma_{0}^{\circ}, \gamma_{0} \in \operatorname{rlv}(\Phi)\right\rangle},
$$

where $f^{u}:=f_{1}^{u_{1}} \ldots f_{r}^{u_{r}}$ as in (2.8.1). Geometrically, the ideal $I(R, \mathfrak{F}, \Phi)$ is the vanishing ideal of the complement $\bar{X} \backslash \widehat{X}$, where $\bar{X}:=\operatorname{Spec}(R)$ and $\widehat{X} \subset \bar{X}$ is the open set introduced in (2.8.1).

Now, let $\left(R_{1}, \mathfrak{F}_{1}, \Phi_{1}\right)$, and $\left(R_{2}, \mathfrak{F}_{2}, \Phi_{2}\right)$ be bunched rings. We set $\bar{X}_{i}:=\operatorname{Spec}\left(R_{i}\right)$, etc. The definition of a morphism is built up in two steps. The first one is

Definition 6.1. By a graded homomorphism of the bunched rings $\left(R_{2}, \mathfrak{F}_{2}, \Phi_{2}\right)$ and $\left(R_{1}, \mathfrak{F}_{1}, \Phi_{1}\right)$, we mean a $\mathbb{K}$-algebra homomorphism $\mu: R_{2} \rightarrow R_{1}$ such that

(i) there is a homomorphism $\widetilde{\mu}: K_{2} \rightarrow K_{1}$ of the respective grading groups with

$$
\mu\left(R_{2, w}\right) \subset R_{1, \widetilde{\mu}(w)} .
$$

(ii) for the respective irrelevant ideals we have

$$
I\left(R_{1}, \mathfrak{F}_{1}, \Phi_{1}\right) \subset \sqrt{\left\langle\mu\left(I\left(R_{2}, \mathfrak{F}_{2}, \Phi_{2}\right)\right)\right\rangle} .
$$

Geometrically such a graded homomorphism is a morphism $\bar{\varphi}: \bar{X}_{1} \rightarrow \bar{X}_{2}$ that maps $\widehat{X}_{1}$ into $\widehat{X}_{2}$ and is equivariant in the sense that $\bar{\varphi}(t \cdot x)=m(t) \cdot \bar{\varphi}(x)$ holds with $m: T_{1} \rightarrow T_{2}$ denoting the homomorphism of tori arising from $\mu: K_{2} \rightarrow K_{1}$.

Definition 6.2. We say that two graded homomorphisms $\mu, \nu$ of the bunched rings $\left(R_{2}, \mathfrak{F}_{2}, \Phi_{2}\right)$ and $\left(R_{1}, \mathfrak{F}_{1}, \Phi_{1}\right)$ are equivalent if there is a homomorphism $\kappa: K_{2} \rightarrow \mathbb{K}^{*}$ such that $\left.\mu\right|_{R_{2, w}}=\left.\kappa(w) \nu\right|_{R_{2, w}}$ always holds. 
From the geometric point of view, two graded homomorphisms are equivalent if and only if the associated equivariant morphisms differ by multiplication with a torus element.

Note that equivalence of graded homomorphisms of bunched rings is compatible with composition, that is, $\mu \circ \nu$ and $\mu^{\prime} \circ \nu^{\prime}$ are equivalent provided both $\mu, \mu^{\prime}$ and $\nu, \nu^{\prime}$ are equivalent. Thus, the following definition of morphism makes the class of bunched rings into a category.

Definition 6.3. A morphism of bunched rings is an equivalence class of graded homomorphisms of bunched rings.

Consider a morphism of the bunched rings $\left(R_{2}, \mathfrak{F}_{2}, \Phi_{2}\right)$ and $\left(R_{1}, \mathfrak{F}_{1}, \Phi_{1}\right)$, and let it be represented by $\mu: R_{2} \rightarrow R_{1}$. Then the associated morphism $\bar{\varphi}: \bar{X}_{1} \rightarrow \bar{X}_{2}$ defines a commutative diagram

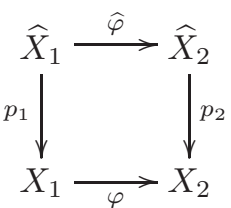

where $X_{i}:=X\left(R_{i}, \mathfrak{F}_{i}, \Phi_{i}\right)$. Note that the morphism $\varphi: X_{1} \rightarrow X_{2}$ does not depend on the choice of the representative $\mu$. Moreover, this construction is compatible with composition, and hence we obtain:

Proposition 6.4. The assignments $(R, \mathfrak{F}, \Phi) \rightsquigarrow X(R, \mathfrak{F}, \Phi)$ and $[\mu] \mapsto \varphi$ define a covariant functor from the category of bunched rings to the category of algebraic varieties.

In general this functor is neither injective nor surjective on the level of morphisms; counterexamples already occur in the case of toric varieties; see 4, Ex. 9.7 and 9.8]. For a more detailed study, the following concept is crucial, compare [2] for the toric case.

Definition 6.5. Let $\varphi: X_{1} \rightarrow X_{2}$ be a dominant morphism. We say that a homomorphism $\eta: \operatorname{WDiv}\left(X_{2}\right) \rightarrow \mathrm{WDiv}\left(X_{1}\right)$ of the respective groups of Weil divisors is a $\varphi$-pullback if

(i) for every principal divisor $D$ on $X_{2}$ we have $\eta(D)=\varphi^{*}(D)$;

(ii) for every effective divisor $D$ on $X_{2}$, the image $\eta(D)$ is again effective;

(iii) for the supports, we have $\operatorname{Supp}(\eta(D)) \subset \varphi^{-1}(\operatorname{Supp}(D))$.

In terms of this notion, we can describe when a given dominant morphism $X_{1} \rightarrow$ $X_{2}$ arises from a morphism of bunched rings. The result generalizes a corresponding statement on toric varieties; see [2, Theorem 2.3].

Theorem 6.6. Let $\varphi: X_{1} \rightarrow X_{2}$ be a dominant morphism. Then the morphisms of the bunched rings $\left(R_{2}, \mathfrak{F}_{2}, X_{2}\right)$ and $\left(R_{1}, \mathfrak{F}_{1}, X_{1}\right)$ that map to $\varphi$ are in one-to-one correspondence with the $\varphi$-pullbacks $\operatorname{WDiv}\left(X_{2}\right) \rightarrow \operatorname{WDiv}\left(X_{1}\right)$.

Before giving the proof of this statement, we list a couple of immediate consequences.

Corollary 6.7. Let $\varphi: X_{1} \rightarrow X_{2}$ be a dominant morphism such that $\varphi^{-1}\left(\operatorname{Sing}\left(X_{2}\right)\right)$ is of codimension at least two in $X_{1}$. Then $\varphi$ arises from a unique morphism of the bunched rings $\left(R_{2}, \mathfrak{F}_{2}, X_{2}\right)$ and $\left(R_{1}, \mathfrak{F}_{1}, X_{1}\right)$. 
This applies in particular to isomorphisms. Thus, we can answer the question of when two bunched rings define isomorphic varieties.

Corollary 6.8. Every isomorphism $X_{1} \rightarrow X_{2}$ arises from a unique isomorphism of the bunched rings $\left(R_{2}, \mathfrak{F}_{2}, X_{2}\right)$ and $\left(R_{1}, \mathfrak{F}_{1}, X_{1}\right)$. In particular, $X_{1}$ and $X_{2}$ are isomorphic if and only if their defining bunched rings are isomorphic.

For a smooth variety $X_{2}$, the following result generalizes [3, Theorem 5.7].

Corollary 6.9. If $X_{2}$ is smooth, then every dominant morphism $X_{1} \rightarrow X_{2}$ arises from a unique morphism of the defining bunched rings.

Proof of Theorem 6.6. We assign to each $\varphi$-pullback $\eta$ : $\operatorname{WDiv}\left(X_{2}\right) \rightarrow \operatorname{WDiv}\left(X_{1}\right)$ a morphism of the bunched rings $\left(R_{2}, \mathfrak{F}_{2}, \Phi_{2}\right)$ and $\left(R_{1}, \mathfrak{F}_{1}, \Phi_{1}\right)$ that induces $\varphi$. This morphism will satisfy the property

$$
p_{1}^{*} \circ \eta=\widehat{\varphi}^{*} \circ p_{2}^{*},
$$

where $p_{i}^{*}: \operatorname{WDiv}\left(X_{i}\right) \rightarrow \operatorname{WDiv}\left(\widehat{X}_{i}\right)$ denotes the pullback discussed in Section 3 and $\widehat{\varphi}: \widehat{X}_{1} \rightarrow \widehat{X}_{2}$ denotes the map arising from any representative $\mu: R_{2} \rightarrow R_{1}$ of our morphism of bunched rings.

First, suppose that $X_{2}$ is a toric variety. In this step, $\varphi: X_{1} \rightarrow X_{2}$ need not be dominant; we only require that $\varphi\left(X_{1}\right)$ intersects the big torus $T_{X_{2}} \subset X_{2}$. Moreover, we replace $\mathrm{WDiv}\left(X_{2}\right)$ with the group of invariant Weil divisors of $X_{2}$. Then it makes again sense to speak of a $\varphi$-pullback.

Recall that $p_{2}: \widehat{X}_{2} \rightarrow X_{2}$ is a Cox construction. Consider the invariant prime divisors $D_{X_{2}}^{i}$ and the corresponding coordinate functions $f_{i}^{2} \in \mathfrak{F}_{2}$ on $\bar{X}_{2}$. Since $R_{1}$ is factorial, we may choose for each $i$ an element $h_{i} \in R_{1}$ with

$$
\operatorname{div}\left(h_{i}\right)=\overline{p_{1}^{*}\left(\eta\left(D_{X_{2}}^{i}\right)\right)} .
$$

As its divisor is invariant, this function is homogeneous, and, by Lemma 5.1, the degree of $h_{i}$ corresponds to the class of $\eta\left(D_{X_{2}}^{i}\right)$ under the identification $K_{1} \cong$ $\mathrm{Cl}\left(X_{1}\right)$. Using condition 6.5 (i), we obtain a family of graded homomorphisms: for $\alpha \in\left(\mathbb{K}^{*}\right)^{r_{2}}$, we set

$$
\mu_{\alpha}: R_{2} \rightarrow R_{1}, \quad f_{i}^{2} \mapsto \alpha_{i} h_{i} .
$$

We now adjust $\alpha$ in such a way that the map $\mathcal{O}\left(T_{\widehat{X}_{2}}\right) \rightarrow \mathbb{K}\left(\widehat{X}_{1}\right)$ induced by $\mu_{\alpha}$ coincides in degree zero with the pullback $\varphi^{*}: \mathcal{O}\left(T_{X_{2}}\right) \rightarrow \mathbb{K}\left(X_{1}\right)$. This amounts to solving the equations

$$
\prod \alpha_{i}^{u_{i}}=p_{1}^{*} \circ \varphi^{*}\left(\prod\left(f_{i}^{2}\right)^{u_{i}}\right) \prod h_{i}^{-u_{i}}
$$

where the vectors $u=\left(u_{1}, \ldots, u_{r_{2}}\right) \in \mathbb{Z}^{r_{2}}$ run through a basis of the space of exponents of the monomials of degree $0 \in K_{2}$. Note that the expressions on the right-hand side are in fact non-vanishing constants, because we have $R_{1}^{*}=\mathbb{K}^{*}$.

Since the vectors $u$ form a matrix of maximal rank, the above system of equations is solvable. Thus, we may fix a solution $\alpha_{0}$. We denote the resulting graded homomorphism by $\mu: R_{2} \rightarrow R_{1}$, and the corresponding map of the spectra by $\bar{\varphi}: \bar{X}_{1} \rightarrow \bar{X}_{2}$. Note that, by construction, any invariant divisor satisfies

$$
\overline{p_{1}^{*} \circ \eta(D)}=\bar{\varphi}^{*}\left(\overline{p_{2}^{*}(D)}\right) \text {. }
$$

If $X_{2}$ is general, then we first construct $\bar{\varphi}: \bar{X}_{1} \rightarrow \bar{Z}_{2}$ for the map $X_{1} \rightarrow Z_{2}$ with an ambient toric variety $Z_{2}$ of $X_{2}$. By our adjustment of $\alpha$, we then see that $\bar{\varphi}$ 
gives an equivariant map $\bar{X}_{1} \rightarrow \bar{X}_{2}$. Note that this map satisfies condition (6.9.2), now for any divisor. Let $\mu: R_{2} \rightarrow R_{1}$ denote the corresponding homomorphism.

We show that $\mu: R_{2} \rightarrow R_{1}$ is a graded homomorphism of bunched rings. For this, we only have to show that the corresponding morphism $\bar{\varphi}: \bar{X}_{1} \rightarrow \bar{X}_{2}$ maps $\widehat{X}_{1}$ into $\widehat{X}_{2}$.

Assume the converse. Then there is an $x \in \widehat{X}_{1}$ with $\bar{\varphi}(x) \in \bar{X}_{2} \backslash \widehat{X}_{2}$. Translated into terms of $\mathfrak{F}_{2}=\left\{f_{1}^{2}, \ldots, f_{s}^{2}\right\}$ and the corresponding divisors $D_{X_{2}}^{i}$ of Proposition 3.6. this means that there is a non-empty set of indices $I \subset\{1, \ldots, s\}$ fulfilling

$$
f_{i}^{2}(\bar{\varphi}(x))=0 \Leftrightarrow i \in I, \quad \bigcap_{i \in I} D_{X_{2}}^{i}=\emptyset .
$$

The first equation shows $x \in \bar{\varphi}^{*}\left(\overline{p_{2}^{*}\left(D_{X_{2}}^{i}\right)}\right)$ for every $i \in I$. Condition (6.9.2) and $x \in \widehat{X}_{1}$ give $x \in p_{1}^{*}\left(\eta\left(D_{X_{2}}^{i}\right)\right)$. But then property 6.5 (iii) leads to a contradiction:

$$
\varphi\left(p_{1}(x)\right) \in \bigcap_{i \in I} D_{X_{2}}^{i}=\emptyset .
$$

The remaining task is to construct the inverse mapping from the set of morphisms of bunched rings inducing $\varphi$ to the set of $\varphi$-pullbacks $\operatorname{WDiv}\left(X_{2}\right) \rightarrow \operatorname{WDiv}\left(X_{1}\right)$. So, let $\mu$ be a graded ring homomorphism inducing $\varphi$, and let $\widehat{\varphi}: \widehat{X}_{1} \rightarrow \widehat{X}_{2}$ be as in (6.3.1).

Consider $D \in \mathrm{WDiv}\left(X_{2}\right)$. Then $E:=\widehat{\varphi}^{*}\left(p_{2}^{*}(D)\right)$ is well defined, because $\varphi$ is dominant. Moreover, this divisor is principal, say $E=\operatorname{div}(h)$ with $h \in \mathbb{K}\left(\widehat{X}_{1}\right)$. Since $E$ is $T_{1}$-invariant, the function $h$ is $T_{1}$-homogeneous, say of degree $w \in K_{1}$.

Since the degrees of the $f_{i}^{1} \in \mathfrak{F}_{1}$ generate $K_{1}$, the function $\widehat{g}:=h / \prod\left(f_{i}^{1}\right)^{u_{i}}$ is of degree zero for suitable $u_{i} \in \mathbb{Z}$. But this implies $\widehat{g}=p_{1}^{*}(g)$ for some $g \in \mathbb{K}\left(X_{1}\right)$. Set

$$
\eta(D):=\operatorname{div}(g)+\sum_{i=1}^{r} u_{i} D_{X_{1}}^{i} .
$$

This gives a well-defined map $\eta: \operatorname{WDiv}\left(X_{2}\right) \rightarrow \mathrm{WDiv}\left(X_{1}\right)$, for which the properties of a $\varphi$-pullback are directly verified. By construction, condition (6.9.1) is satisfied.

To conclude the proof, we have to check that the two assignments are inverse to each other. Starting with a $\varphi$-pullback, constructing the associated morphism of bunched rings, and then returning to the $\varphi$-pullbacks gives nothing new, because condition (6.9.1) determines the $\varphi$-pullbacks. For the other way around, we may argue similarly: the class of a graded homomorphism is fixed by condition (6.9.1).

\section{Picard Group, Semiample, ANd Ample Cone}

We present explicit descriptions of the Picard group, the semiample and the ample cone of the variety $X$ arising from a bunched ring $(R, \mathfrak{F}, \Phi)$. The descriptions are given in terms of the isomorphism $\overline{\mathfrak{D}}_{X}: K \rightarrow \mathrm{Cl}(X)$ provided in Lemma 5.1 they generalize the corresponding results on toric varieties of [4, Sec. 10].

Proposition 7.1. Let $X$ be the variety arising from a bunched ring $(R, \mathfrak{F}, \Phi)$, and let $(E \stackrel{Q}{\longrightarrow} K, \gamma)$ be the projected cone associated to $\mathfrak{F}$. Then, in $K \cong \mathrm{Cl}(X)$, the Picard group is given by

$$
\operatorname{Pic}(X)=\bigcap_{\gamma_{0} \in \operatorname{cov}(\Phi)} Q\left(\operatorname{lin}\left(\gamma_{0}\right) \cap E\right) .
$$


Proof. We have to figure out when $w \in K$ represents a Cartier divisor. According Proposition 5.3, this is the case if and only if

$$
w \in \bigcap_{\gamma_{0} \in \operatorname{rlv}(\Phi)} Q\left(\operatorname{lin}\left(\gamma_{0}\right) \cap E\right) .
$$

Now recall that $\operatorname{cov}(\Phi)$ is a subset of $\operatorname{rlv}(\Phi)$, and that for any element $\gamma_{0} \in \operatorname{rlv}(\Phi)$, there is a face $\gamma_{1} \preceq \gamma_{0}$ with $\gamma_{1} \in \operatorname{cov}(\Phi)$. So, it suffices to take the intersection over $\operatorname{cov}(\Phi)$.

We now describe some cones of rational divisor classes on $X$ that are of general interest. First we consider the cone $\operatorname{Eff}(X) \subset \mathrm{Cl}_{\mathbb{Q}}(X)$ generated by the classes of the effective divisors and the moving cone $\operatorname{Mov}(X) \subset \mathrm{Cl}_{\mathbb{Q}}(X)$. For the definition of the latter, recall that the base locus of a divisor $D \in \operatorname{WDiv}(X)$ is

$$
\bigcap_{f \in \Gamma(X, \mathcal{O}(D))} Z(f), \quad \text { where } Z(f):=\operatorname{Supp}(\operatorname{div}(f)+D) .
$$

Then the moving cone $\operatorname{Mov}(X) \subset \mathrm{Cl}_{\mathbb{Q}}(X)$ is the cone generated by the classes of those divisors, the base loci of which are of codimension at least two. Our result generalizes corresponding statements in the toric case; for the description of the toric moving cone see, e.g., [16, Prop. 3.1].

Proposition 7.2. Let $(R, \mathfrak{F}, \Phi)$ be a bunched ring with associated projected cone $(E \stackrel{Q}{\longrightarrow} K, \gamma)$, and let $X:=X(R, \mathfrak{F}, \Phi)$.

(i) Let $e_{1}, \ldots, e_{r}$ be the primitive generators of $\gamma$. Then, in $K_{\mathbb{Q}}=\mathrm{Cl}_{\mathbb{Q}}(X)$, the effective cone of $X$ is given by

$$
\operatorname{Eff}(X)=\operatorname{cone}\left(Q\left(e_{1}\right), \ldots, Q\left(e_{r}\right)\right) .
$$

(ii) Let $\gamma_{1}, \ldots, \gamma_{r}$ denote the facets of $\gamma$. Then the moving cone of $X$ is of full dimension in $K_{\mathbb{Q}}=\mathrm{Cl}_{\mathbb{Q}}(X)$, and it is given by

$$
\operatorname{Mov}(X)=Q\left(\gamma_{1}\right) \cap \ldots \cap Q\left(\gamma_{r}\right) .
$$

Proof. A divisor $D$ is linearly equivalent to an effective one, if and only if $D$ admits a global section. Since $R$ is the total coordinate ring of $X$, the latter holds, if and only if we have $R_{w} \neq 0$ for the degree $w \in K$ corresponding to the class of $D$. In other words, the effective cone in $K$ is precisely the grading cone of $R$. This proves (i).

To verify (ii), let $D$ be any effective divisor on $X$. We have to show that $D$ has no fixed components if and only if its corresponding degree $w$ lies in every $Q\left(\gamma_{i}\right)$. According to (i), we may assume that $D$ is a non-negative linear combination of the divisors $D_{X}^{i}$ introduced in Proposition 3.6.

Now, by definition, $D_{X}^{i}$ is a fixed component of $D$ if and only if for every section $h \in \Gamma(X, \mathcal{O}(D))$, the prime divisor $D_{X}^{i}$ occurs in $\left.\operatorname{Supp}(\operatorname{div}(h)+D)\right)$. This holds if and only if the pullback divisors with respect to $p_{X}: \widehat{X} \rightarrow X$ as defined in Section 3 satisfy

$$
p_{X}^{*}\left(D_{X}^{i}\right) \leq p_{X}^{*}(D)+\operatorname{div}\left(p_{X}^{*}(h)\right) .
$$

Using factoriality of $R$, we can conclude that $D_{X}^{i}$ is a fixed component of $D$ if and only if $f_{i}$ divides every $f \in R_{w}$, where $w \in K$ denotes the class of $D$. But the latter can be expressed in terms of degrees: it holds if and only if the class $w \in K$ cannot be written as a non-negative linear combination of the $w_{j}=Q\left(e_{j}\right)$ with $j \neq i$. 
In other words, $D_{X}^{i}$ is not a fixed component of $D$ if and only if $D$ corresponds to an element of cone $\left(Q\left(e_{j}\right) ; j \neq i\right)$. This verifies the explicit description of the moving cone. The fact that $\operatorname{Mov}(X)$ is of full dimension is then an immediate consequence of Lemma 1.7.

Next we describe the semiample cone $\operatorname{Sample}(X)$ and the ample cone $\operatorname{Ample}(X)$ in $\mathrm{Cl}_{\mathbb{Q}}(X)$. Recall that, by definition, the semiample cone is generated by the classes of the divisors having an empty base locus. Moreover, the ample cone is generated by the classes of $\operatorname{divisors} D \in \operatorname{WDiv}(X)$ that provide a cover of $X$ by affine open sets of the form $X \backslash Z(f)$ with $f \in \Gamma(X, \mathcal{O}(D))$.

The descriptions of the semiample and the ample cone can be done purely in terms of the $\mathfrak{F}$-bunch $\Phi$.

Theorem 7.3. Let $X$ be the variety arising from a bunched ring $(R, \mathfrak{F}, \Phi)$ with grading lattice $K$. Then, in $K_{\mathbb{Q}} \cong \mathrm{Cl}_{\mathbb{Q}}(X)$, the semiample and the ample cone are given by

$$
\operatorname{Sample}(X)=\bigcap_{\tau \in \Phi} \tau, \quad \operatorname{Ample}(X)=\bigcap_{\tau \in \Phi} \tau^{\circ} .
$$

Proof. Consider the embedding $X \subset Z:=Z(R, \mathfrak{F}, \Phi)$ into the minimal toric variety presented in Proposition 3.7. Recall that every closed $T_{Z}$-orbit has non-trivial intersection with $X$. Moreover, $X \subset Z$ defines a pullback isomorphism $\mathrm{Cl}(Z) \rightarrow$ $\mathrm{Cl}(X)$. This is due to Proposition 5.2 and the fact $Z$ is an open toric subvariety of any ambient toric variety of $X$.

We claim that the semiample cone $\operatorname{Sample}(Z)$ is mapped onto Sample $(X)$. Indeed, given a class $[D] \in \operatorname{Sample}(X)$, this class extends to a class $\left[D^{\prime}\right] \in \mathrm{Cl}(Z)$, represented by some $T_{Z}$-invariant divisor $D^{\prime}$ on $Z$. Since the base locus of $D^{\prime}$ is $T_{Z}$-invariant and $X$ meets every closed $T_{Z}$-orbit, it follows that $D^{\prime}$ is semiample. This proves $\operatorname{Sample}(Z)=\operatorname{Sample}(X)$. Thus, we only have to verify

$$
\operatorname{Sample}(Z)=\bigcap_{\tau \in \Phi} \tau \text {. }
$$

Consider the projected cone $(E \stackrel{Q}{\longrightarrow} K, \gamma)$ associated to $\mathfrak{F} \subset R$, and its dual projected cone $(F \stackrel{P}{\longrightarrow} N, \delta)$. We denote by $\widehat{\Delta}$ the fan in $F$ having $\gamma_{0}^{*}$, where $\gamma_{0} \in \operatorname{cov}(\Phi)$, as its maximal cones. Then, by definition, the fan $\Delta:=\Delta(R, \mathfrak{F}, \Phi)$ of $Z$ has the images $P\left(\delta_{0}\right)$, where $\delta_{0} \in \widehat{\Delta}^{\text {max }}$, as its maximal cones.

In the sequel, we follow the lines of the proof of [4, Theorem 10.2]. Let $e_{\varrho}$ denote the primitive generator of $\delta$, which is mapped to the primitive generator of $\varrho \in \Delta^{(1)}$. Then [4, Prop. 10.1 and 10.6], provide an isomorphism

$$
\mathfrak{D}_{Z}: E \rightarrow \operatorname{WDiv}^{T_{Z}}(Z), \quad \widehat{w} \mapsto \sum_{\varrho \in \Delta^{(1)}}\left\langle\widehat{w}, e_{\varrho}\right\rangle \overline{T_{Z} \cdot z_{\varrho}} .
$$

Next recall from [11 that an invariant divisor $D$ on $Z$ is semiample if and only if it is described by a convex conewise linear function $\left(u_{\sigma}\right)$ living on the support of the fan $\Delta$. Here convexity means that $u_{\sigma}-u_{\sigma^{\prime}}$ is non-negative on $\sigma \backslash \sigma^{\prime}$ for any two $\sigma, \sigma^{\prime} \in \Delta^{\max }$. Here we do not restrict ourselves to integral linear forms $u_{\sigma}$, but we also admit rational ones.

Now, let $w \in K$ represent a semiample class. This class comes from a semiample rational divisor $D=\mathfrak{D}_{Z}(\widehat{w})$ with $\widehat{w} \in E_{\mathbb{Q}}$ such that $w=Q(\widehat{w})$. Let $\left(u_{\sigma}\right)$ be a 
convex function describing $D$. We have to interpret $\left(u_{\sigma}\right)$ in terms of the fan $\widehat{\Delta}$. For $\sigma \in \Delta^{\text {max }}$, let $\widehat{\sigma} \in \widehat{\Delta}$ be the (unique) cone with $P(\widehat{\sigma})=\sigma$.

Then, for $\ell_{\sigma}:=\widehat{w}-P^{*}\left(u_{\sigma}\right)$, each $\ell_{\sigma^{\prime}}-\ell_{\sigma}$ is non-negative on $\widehat{\sigma} \backslash \widehat{\sigma}^{\prime}$. Since $\ell_{\sigma} \in \widehat{\sigma}^{\perp}$ holds, this is equivalent to the non-negativity of $\ell_{\sigma^{\prime}}$ on every $\widehat{\sigma} \backslash \widehat{\sigma}^{\prime}$. Since all rays of the cone $\delta$ occur in the fan $\widehat{\Delta}$, the latter is valid if and only if $\ell_{\sigma} \in \widehat{\sigma}^{*}$ holds for all $\sigma$. This in turn implies that for every $\sigma \in \Delta^{\max }$ we have

$$
w=Q(\widehat{w})=Q\left(\ell_{\sigma}\right) \in Q\left(\widehat{\sigma}^{*}\right) .
$$

By the definition of the fan $\Delta$, the cones of $\Phi$ are the minimal ones among the images $Q\left(\widehat{\sigma}^{*}\right)$, where $\sigma \in \Delta^{\max }$. Consequently, $w$ lies in the intersection over the cones of $\Phi$.

Conversely, if $w$ belongs to the intersection of all cones in $\Phi$, then $w$ is as well an element of every image $Q\left(\widehat{\sigma}^{*}\right)$ with $\sigma \in \Delta^{\max }$. In particular, we find for every $\sigma \in \Delta^{\max }$ an $\ell_{\sigma} \in \widehat{\sigma}^{*}$ mapping to $w$. Reversing the above arguments, we see that $u_{\sigma}:=\widehat{w}-\ell_{\sigma}$ is a convex support function describing the representative $D:=\mathfrak{D}_{Z}(\widehat{w})$ of the class $\overline{\mathfrak{D}}_{Z}(w)$.

The description of the ample cone is obtained in an analogous manner, and therefore is left to the reader. For the equality $\operatorname{Ample}(Z)=\operatorname{Ample}(X)$ we just note that any $T_{Z}$-invariant divisor has a $T_{Z}$-invariant ample locus, i.e., the set of points admitting an affine neighbourhood $Z_{f}$ with a global section $f$ of $\mathcal{O}(D)$.

For a variety $X$ with finitely generated free divisor class group, the Picard group $\operatorname{Pic}(X)$ is equal to the Néron-Severi group $N^{1}(X)$. In the associated vector space $N_{\mathbb{Q}}^{1}(X)=\operatorname{Pic}_{\mathbb{Q}}(X)$, one studies the numerically effective cone, i.e., the cone generated by the classes of the Cartier divisors having non-negative intersection with all (effective) curves.

For projective varieties with free finitely generated divisor class group and finitely generated total coordinate ring, we obtain as an immediate consequence of the preceding results and Kleiman's ampleness criterion that the numerically effective cone and the semiample cone coincide; compare [17, Prop. 1.11] for the $\mathbb{Q}$-factorial case.

Corollary 7.4. Let $X$ be a normal projective variety with free finitely generated divisor class group and finitely generated total coordinate ring.

(i) The ample and the semiample cone of $X$ are of full dimension in $\operatorname{Pic}_{\mathbb{Q}}(X)$.

(ii) The ample cone of $X$ is the relative interior of the semiample cone of $X$.

(iii) The numerically effective cone and the semiample cone of $X$ coincide.

Proof. We may assume that $X$ arises from a bunched ring $(R, \mathfrak{F}, \Phi)$. Since $X$ is projective, it admits ample divisors. Thus, Theorem 7.3 tells us that the intersection of all relative interiors $\tau^{\circ}$, where $\tau \in \Phi$, is non-empty. Consequently, Proposition 7.1 and, once more, Theorem 7.3 give the first two assertions; the first one is also a special case of [19, Thm. IV.2.1]. The third assertion follows from the the second one and the fact that for projective varieties, the numerically effective cone is the closure of the ample cone; see [19, Thm. IV.2.1].

The Mori cone is the cone $\operatorname{Mori}(X)$ in the dual space of $N_{\mathbb{Q}}^{1}(X)$ generated by the numerical equivalence classes of effective curves. In our setup, the Mori cone lives in the dual vector space of $\operatorname{Pic}_{\mathbb{Q}}(X)$, and we obtain an explicit description for it. 
Corollary 7.5. Let $X$ be a projective variety arising from a bunched ring $(R, \mathfrak{F}, \Phi)$. Then, in $\operatorname{Pic}_{\mathbb{Q}}(X)^{*}$, the Mori cone of $X$ is the strictly convex cone given by

$$
\operatorname{Mori}(X)=\sum_{\tau \in \Phi}\left(\operatorname{Pic}(X)_{\mathbb{Q}} \cap \tau\right)^{\vee}
$$

Proof. The Mori cone is dual to the numerically effective cone in $\operatorname{Pic}_{\mathbb{Q}}(X)$, by definition of the latter. Hence Corollary 7.4 Proposition 7.1, and Theorem 7.3 give the assertion.

Finally, we note that the proofs of the results of this section show that the various cones of divisor classes are inherited from suitable toric ambient varieties.

Remark 7.6. Let $X:=X(R, \mathfrak{F}, \Phi)$ be the variety arising from a bunched ring $(R, \mathfrak{F}, \Phi)$.

(i) For any toric ambient variety $Z$ of $X$ in the sense of Proposition 3.2 we have

$$
\operatorname{Eff}(X)=\operatorname{Eff}(Z), \quad \operatorname{Mov}(X)=\operatorname{Mov}(Z) .
$$

(ii) For the minimal toric ambient variety $Z:=Z(R, \mathfrak{F}, \Phi)$ of $X$ we have

$$
\operatorname{Sample}(X)=\operatorname{Sample}(Z), \quad \operatorname{Ample}(X)=\operatorname{Ample}(Z) .
$$

It should be mentioned that the second statement may become false if one enlarges the minimal toric ambient variety, e.g., if one makes it $A_{2}$-maximal; see [29] and Proposition 11.6 .

\section{Projectivity and Fano Criteria}

In this section, we apply the description of the ample cone given in the previous section to obtain projectivity and Fano criteria. The first result is an effective version of the Kleiman-Chevalley Criterion. In our setup, it sharpens considerations performed in [23, p. 307].

Theorem 8.1. Let $X$ be a (normal) $A_{2}$-maximal $\mathbb{Q}$-factorial variety with $\mathcal{O}(X)=$ $\mathbb{K}$, free divisor class group of rank $k$ and finitely generated total coordinate ring. If any collection $x_{1}, \ldots, x_{k} \in X$ admits a common affine neighbourhood in $X$, then $X$ is projective.

For the proof, and also for later purposes, we need the following characterization of existence of non-trivial global functions.

Proposition 8.2. Let $X$ arise from a bunched ring $(R, \mathfrak{F}, \Phi)$, and let $(E \stackrel{Q}{\longrightarrow} K, \gamma)$ be the projected cone associated to $\mathfrak{F}$. Then the following statements are equivalent:

(i) $\mathcal{O}(X)=\mathbb{K}$,

(ii) the cone $Q(\gamma)$ is strictly convex, and $Q\left(e_{i}\right) \neq 0$ holds for each primitive generator $e_{i}$ of $\gamma$.

Proof. Choose a bunch $\Theta$ extending $\Phi$, and consider the corresponding ambient toric variety $Z$ of $X$. We claim that $\mathcal{O}(X)=\mathbb{K}$ is equivalent to $\mathcal{O}(Z)=\mathbb{K}$. In terms of $\bar{X}=\operatorname{Spec}(R)$ and $\bar{Z}=\operatorname{Spec}(\mathbb{K}[E \cap \gamma])$, and the torus $T=\operatorname{Spec}(\mathbb{K}[K])$, this means to show that

$$
\mathcal{O}(\bar{X})^{T}=\mathbb{K} \Leftrightarrow \mathcal{O}(\bar{Z})^{T}=\mathbb{K} .
$$

The implication " $\Leftarrow$ " is easy. The reverse implication follows from the fact that $\bar{X}$ meets the big torus and every coordinate hyperplane of $\bar{Z}=\mathbb{K}^{r}$ : if $\bar{Z}$ admits a 
non-constant $T$-invariant function, then it admits also a non-constant $T$-invariant monomial. This in turn restricts to a non-constant function on $\bar{X}$.

Thus we verified that $\mathcal{O}(X)=\mathbb{K}$ is equivalent to $\mathcal{O}(Z)=\mathbb{K}$. But the latter property was characterized in [4, Proposition 8.4] in terms of the bunch $\Theta$ : it is valid if and only if $Q$ contracts no ray of $\gamma$, and $Q(\gamma)$ is strictly convex.

Another preparatory result concerns the question of whether or not an $A_{2^{-}}$ maximal variety $X$ with finitely generated free divisor class group and finitely generated total coordinate ring is necessarily complete if it satisfies $\mathcal{O}(X)=\mathbb{K}$. First note that, in general, the answer to this question is no.

In fact, there exist examples of non-complete fans as in Corollary 4.5 the support of which generates the whole ambient vector space as a cone; see [10]. The corresponding toric varieties are $A_{2}$-maximal and non-complete. However, such varieties can never be quasiprojective, as we will now see.

Proposition 8.3. Let $X$ be an $A_{2}$-maximal variety with free finitely generated divisor class group and finitely generated total coordinate ring. If $X$ is quasiprojective and $\mathcal{O}(X)=\mathbb{K}$ holds, then $X$ is even projective.

Proof. We may assume that $X$ arises from a bunched $\operatorname{ring}(R, \mathfrak{F}, \Phi)$. Then $X$ is in particular a good quotient of an open subset $\widehat{X}$ of $\bar{X}:=\operatorname{Spec}(R)$ by the action of a torus $T$ with $\operatorname{dim}(T)$ equal to $\operatorname{dim}(\bar{X})-\operatorname{dim}(X)$.

Since $X=\widehat{X} / / T$ is quasiprojective, it admits an open embedding into the quotient $X^{\prime}=\widehat{X}^{\prime}(w) / / T$ of the set $\widehat{X}^{\prime} \subset \widehat{X}$ of semistable points of some $T$ linearization of the trivial bundle over $\bar{X}$; compare [26, Converse 1.13]. The assumption $\mathcal{O}(\bar{X})^{T}=\mathcal{O}(X)=\mathbb{K}$ implies that the quotient space $X^{\prime}$ is projective.

Since $\widehat{X}$ has a complement at least two in $\bar{X}$, the same holds for $\widehat{X}$ in $\widehat{X}^{\prime}$, and hence for $X$ in $X^{\prime}$. By $A_{2}$-maximality, we have $X=X^{\prime}$, and hence $X$ is projective.

Proof of Theorem 8.1 . If $X$ is as in the assertion, then $X=X(R, \mathfrak{F}, \Phi)$ holds with a bunched ring $(R, \mathfrak{F}, \Phi)$. According to Proposition 8.3 and Theorem 7.3 , we have to show that the intersection over all relative interiors $\tau^{\circ}$, where $\tau \in \Phi$, is non-empty. As usual, let $(E \stackrel{Q}{\longrightarrow} K, \gamma)$ denote the projected cone associated to $\mathfrak{F}$.

We first perform a reduction step. Consider the convex hull $\vartheta$ of all $\tau \in \Phi$. Then Proposition 8.2 tells us that $\vartheta$ is a strictly convex cone, because we assumed $\mathcal{O}(X)=\mathbb{K}$. Thus, choosing a $w \in \vartheta^{\circ}$ and a suitable affine hyperplane $H \subset K_{\mathbb{Q}}$ orthogonal to $w$, we obtain for every $\tau \in \Phi$ a non-empty polytope $B(\tau):=H \cap \tau$. By Corollary [5.4, we have $\operatorname{dim}(\tau)=k$, so $B(\tau)$ is of dimension $k-1$.

Clearly it suffices to show that the intersection of all $B(\tau)^{\circ}$, where $\tau \in \Phi$, is nonempty. According to Helly's Theorem, see [24, Satz I.7.1], the latter holds if any intersection $B\left(\tau_{1}\right)^{\circ} \cap \ldots \cap B\left(\tau_{k}\right)^{\circ}$ is nonempty. Thus is suffices to that for any collection $\tau_{1}, \ldots, \tau_{k}$, the intersection of their relative interiors is nonempty.

Choose a bunch $\Theta$ extending $\Phi$, and consider the corresponding ambient toric variety $Z$ of $X$. Given $\tau_{1}, \ldots, \tau_{k}$ as before, choose $\gamma_{i} \in \operatorname{rlv}(\Phi)$ with $Q\left(\gamma_{i}\right)=\tau_{i}$, and let $Z^{\prime} \subset Z$ be the associated open toric subvariety, i.e.,

$$
Z^{\prime}=\bigcup_{i=1}^{k} \operatorname{Spec}\left(\mathbb{K}\left[\operatorname{ker}(Q) \cap \gamma_{i}\right]\right)
$$


We claim that $Z^{\prime}$ is quasiprojective. First note that $Z^{\prime}$ is $\mathbb{Q}$-factorial. Moreover, the big torus $T^{\prime} \subset Z^{\prime}$ has at most $k$ closed orbits in $Z^{\prime}$, and any such orbit contains a point of $X$. Thus we find an affine open subset $W \subset Z^{\prime}$ that intersects every closed $T^{\prime}$-orbit of $Z^{\prime}$.

Since $Z^{\prime}$ is also $\mathbb{Q}$-factorial, the complement $Z^{\prime} \backslash W$ is the support of a Cartier divisor $D^{\prime}$ on $Z^{\prime}$. Since the translates $t^{\prime} \cdot W$ cover $Z^{\prime}$, and $D^{\prime}$ is linearly equivalent to an invariant divisor, we can conclude that $D^{\prime}$ is ample. Thus $Z^{\prime}$ is quasiprojective. As the toric part of the proof of Theorem 7.3 shows, this implies that $\tau_{1}^{\circ} \cap \ldots \cap \tau_{k}^{\circ}$ is non-empty.

Corollary 8.4. Every (normal) $\mathbb{Q}$-factorial $A_{2}$-variety $X$ with $\mathcal{O}(X)=\mathbb{K}$, free divisor class group of rank two and finitely generated total coordinate ring is quasiprojective.

Proof. Assertion 4.2 a) and Theorem 4.2 tell us that $X$ is an open subset of some $A_{2}$-maximal variety with the same properties. Thus, Theorem 8.1 gives the desired result.

In this corollary, the assumption that $X$ is an $A_{2}$-variety is essential: one obtains complete non-projective threefolds with divisor class group isomorphic to $\mathbb{Z}^{2}$ as geometric quotient spaces of a certain $\mathbb{K}^{*}$-action on the Grassmannian $G(2,4)$; see for example [5] and [28].

Our next aim is to give some Fano criteria for the variety $X$ arising from a bunched $\operatorname{ring}(R, \mathfrak{F}, \Phi)$. For this, we first have to determine the canonical divisor class of $X$. This can be easily done if $X$ is a complete intersection in some of its ambient toric varieties.

Let $(E \stackrel{Q}{\longrightarrow} K, \gamma)$ be the projected cone associated to $\mathfrak{F}$. Denote the primitive generators of $\gamma$ by $e_{1}, \ldots, e_{r}$, and set $w_{i}:=Q\left(e_{i}\right)$.

Proposition 8.5. Suppose that the relations of $\mathfrak{F}$ are generated by $K$-homogeneous polynomials $g_{1}, \ldots, g_{d} \in \mathbb{K}\left[T_{1}, \ldots, T_{r}\right]$, where $d:=r-\operatorname{rank}(K)-\operatorname{dim}(X)$. Then, in $K \cong \mathrm{Cl}(X)$, the canonical divisor class of $X$ is given by

$$
D_{X}^{c}=\sum_{i=1}^{d} \operatorname{deg}\left(g_{i}\right)-\sum_{i=1}^{r} w_{i} .
$$

Proof. Consider the embedding $X \rightarrow Z$ into any ambient toric variety $Z$ of $X$. Then Proposition 5.7 tells us that the respective embeddings of the smooth loci fit into a commutative diagram

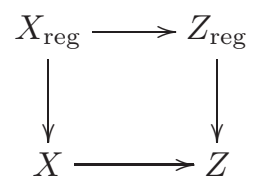

Note that all maps induce isomorphisms on the respective divisor class groups. Moreover, the restrictions of the canonical divisors $K_{X}$ and $K_{Z}$ of $X$ and $Z$ give the canonical divisors of $X_{\text {reg }}$ and $Z_{\text {reg }}$, respectively; see for example [25, p. 164]. Thus, we may assume that $X$ and its ambient toric variety $Z$ are smooth.

Let $\mathcal{I} \subset \mathcal{O}_{Z}$ be the ideal sheaf of $X$. Then the normal sheaf of $X$ in $Z$ is the rank $d$ locally free sheaf $\mathcal{N}_{X}:=\left(\mathcal{I} / \mathcal{I}^{2}\right)^{*}$, and a canonical bundle on $X$ can be obtained 
as follows (see for example [13, Prop. II.8.20]):

$$
\mathcal{K}_{X}=\left.\mathcal{K}_{Z}\right|_{X} \otimes\left(\bigwedge^{d} \mathcal{N}_{X}\right)
$$

Choose a cover of $Z$ by open subsets $U_{i}$ such that $\mathcal{I} / \mathcal{I}^{2}$ is free over $U_{i}$. Then the $g_{l}$ generate the relations of the $f_{j}$ over $\widehat{U}_{i}:=p_{Z}^{-1}\left(U_{i}\right)$. Thus, after suitably refining the cover, we find functions $h_{i l} \in \mathcal{O}^{*}\left(\widehat{U}_{i}\right)$ of degree $\operatorname{deg}\left(g_{l}\right)$ such that $\mathcal{I} / \mathcal{I}^{2}\left(U_{i}\right)$ is generated by $g_{1} / h_{i 1}, \ldots, g_{d} / h_{i d}$. Therefore over $U_{i}$, the $d$-th exterior power of $\mathcal{I} / \mathcal{I}^{2}$ is generated by the function

$$
\frac{g_{1}}{h_{i 1}} \wedge \cdots \wedge \frac{g_{d}}{h_{i d}}
$$

Now, Lemma 5.1 tells us that the class of $\bigwedge^{d} \mathcal{I} / \mathcal{I}^{2}$ in $K$ is minus the sum of the degrees of the $g_{j}$. As $\bigwedge^{d} \mathcal{N}_{X}$ is the dual sheaf, its class is $\operatorname{deg}\left(g_{1}\right)+\ldots+\operatorname{deg}\left(g_{l}\right)$. Furthermore, from [4, Section 10] we know that the class of the canonical divisor of $Z$ in $K$ is given by $-\left(w_{1}+\ldots+w_{r}\right)$. Putting all together we arrive at the assertion.

A variety is said to be $(\mathbb{Q}$-) Gorenstein if (some multiple of) its anticanonical divisor is Cartier. In our setting, we obtain

Corollary 8.6. In the setting of Proposition 8.5, the variety $X$ is

(i) $\mathbb{Q}$-Gorenstein if and only if

$$
\sum_{i=1}^{r} w_{i}-\sum_{j=1}^{d} \operatorname{deg}\left(g_{j}\right) \in \bigcap_{\tau \in \Phi} \operatorname{lin}(\tau)
$$

(ii) Gorenstein if and only if

$$
\sum_{i=1}^{r} w_{i}-\sum_{j=1}^{d} \operatorname{deg}\left(g_{j}\right) \in \bigcap_{\gamma_{0} \in \operatorname{rlv}(\Phi)} Q\left(\operatorname{lin}\left(\gamma_{0}\right) \cap E\right) .
$$

Finally, as announced, we determine, when $X$ is a $(\mathbb{Q}$-)Fano variety, i.e., when (some multiple of) its anticanonical divisor is ample.

Corollary 8.7. In the situation of Proposition 8.5, the variety $X$ is

(i) $\mathbb{Q}$-Fano if and only if we have

$$
\sum_{i=1}^{r} w_{i}-\sum_{j=1}^{d} \operatorname{deg}\left(g_{j}\right) \in \bigcap_{\tau \in \Phi} \tau^{\circ},
$$

(ii) Fano if and only if we have

$$
\sum_{i=1}^{r} w_{i}-\sum_{j=1}^{d} \operatorname{deg}\left(g_{j}\right), \in \bigcap_{\tau \in \Phi} \tau^{\circ} \cap \bigcap_{\gamma_{0} \in \operatorname{rlv}(\Phi)} Q\left(\operatorname{lin}\left(\gamma_{0}\right) \cap E\right) .
$$

\section{INTRINSIC QUADRICS I}

In the following two sections, we consider varieties that have a total coordinate ring defined by a single non-trivial relation. We focus on the case of a quadratic relation, and study these varieties in terms of their bunched rings. For example, we shall classify the smooth complete varieties of this type having a divisor class group of rank two. 
Definition 9.1. By an intrinsic hypersurface, we mean a normal $A_{2}$-maximal variety $X$ with $\mathcal{O}^{*}(X)=\mathbb{K}^{*}$ and free finitely generated divisor class group $K:=\mathrm{Cl}(X)$ such that the total coordinate ring of $X$ admits a representation

$$
\mathcal{R}(X) \cong \mathbb{K}\left[T_{1}, \ldots, T_{r}\right] /\langle g\rangle, \quad \text { with } \mathbb{K}\left[T_{1}, \ldots, T_{r}\right]=\bigoplus_{w \in K} \mathbb{K}\left[T_{1}, \ldots, T_{r}\right]_{w},
$$

where the relation $g$ is homogeneous and non-trivial, and the classes of $T_{1}, \ldots, T_{r}$ are homogeneous pairwise non-associated prime elements, forming a system of generators of minimal length for $\mathcal{R}(X)$.

By Theorem 4.2, every intrinsic hypersurface is of the form $X=X(R, \mathfrak{F}, \Phi)$ with a bunched ring $(R, \mathfrak{F}, \Phi)$. Moreover, if $\mathfrak{F} \subset R$ is a system of generators of minimal length, then Proposition 3.2 says that $X$ is a hypersurface in each of its ambient toric varieties; this explains the name.

We begin with some general observations. Let $R$ be any factorial ring satisfying $R^{*}=\mathbb{K}^{*}$, and suppose that there is a representation

$$
R=\mathbb{K}\left[T_{1}, \ldots, T_{r}\right] /\langle g\rangle
$$

for some non-trivial $g \in \mathbb{K}\left[T_{1}, \ldots, T_{r}\right]$. The following criteria are often helpful for deciding whether or not the class of $T_{i}$ is prime in $R$.

Remark 9.2. Let $g \in \mathbb{K}\left[T_{1}, \ldots, T_{r}\right]$ be such that $R:=\mathbb{K}\left[T_{1}, \ldots, T_{r}\right] /\langle g\rangle$ is factorial.

(i) The class of $T_{i}$ is prime in $R$, if and only if $g\left(T_{1}, \ldots, T_{i-1}, 0, T_{i+1}, \ldots, T_{r}\right)$ is irreducible in $\mathbb{K}\left[T_{1}, \ldots, T_{i-1}, T_{i+1}, \ldots, T_{r}\right]$.

(ii) If $R$ admits an $\mathbb{N}$-grading such that $R_{0}^{*}=\mathbb{K}^{*}$ and $\operatorname{deg}\left(T_{i}\right)= \pm 1$ hold, then the class of $T_{i}$ is prime in $R$.

In the sequel, we will assume that $R$ is as in Definition 9.1. That means that $\mathbb{K}\left[T_{1}, \ldots, T_{r}\right]$ is graded by some lattice $K$, the $T_{i}$ and $g$ are homogeneous, and the classes $f_{i} \in R$ of $T_{i}$ are pairwise non-associated prime elements, forming a system of generators of minimal length for $R$. Moreover, we require that the degrees

$$
w_{i}:=\operatorname{deg}\left(f_{i}\right)=\operatorname{deg}\left(T_{i}\right)
$$

generate $K$ as a lattice. Let $(E \stackrel{Q}{\longrightarrow} K, \gamma)$ denote the projected cone associated to $\mathfrak{F}$. Thus, $E=\mathbb{Z}^{r}$ and $Q\left(e_{i}\right)=w_{i}$ hold, and $\gamma \subset E_{\mathbb{Q}}$ is the positive orthant. We want to determine the $\mathfrak{F}$-faces for $\mathfrak{F}:=\left\{f_{1}, \ldots, f_{r}\right\}$. For a given face $\gamma_{0} \preceq \gamma$, consider the number

$$
\nu\left(\gamma_{0}\right):=\#\left(u \in \gamma_{0} \cap E ; \alpha_{u} \neq 0\right), \quad \text { where } g=\sum \alpha_{u} T^{u} .
$$

Proposition 9.3. A face $\gamma_{0} \preceq \gamma$ is an $\mathfrak{F}$-face if and only if $\nu\left(\gamma_{0}\right)$ differs from one.

Proof. We treat as an example a face of the form $\gamma_{0}:=\operatorname{cone}\left(e_{1}, \ldots, e_{s}\right)$. Consider the polynomial

$$
h\left(T_{1}, \ldots, T_{s}\right):=g\left(T_{1}, \ldots, T_{s}, 0, \ldots, 0\right) .
$$

Then, by Remark 2.2, the cone $\gamma_{0}$ is an $\mathfrak{F}$-face if and only if there is a point $z \in \mathbb{K}^{r}$ with

$$
z_{1}, \ldots, z_{s} \in \mathbb{K}^{*}, \quad z_{s+1}, \ldots, z_{r}=0, \quad g(z)=h\left(z_{1}, \ldots, z_{s}\right)=0 .
$$

Observe that the polynomial $h$ is a sum of exactly $\nu\left(\gamma_{0}\right)$ (non-trivially scaled) monomials. Thus, the desired $z_{1}, \ldots, z_{s}$ exist if and only if $\nu\left(\gamma_{0}\right)$ differs from one. 
We now specialize to the case that $R$ has a defining relation $q:=g \in \mathbb{K}\left[T_{1}, \ldots, T_{r}\right]$ of degree two. Let $\Phi$ be an $\mathfrak{F}$-bunch in the projected cone $(E \stackrel{Q}{\longrightarrow} K, \gamma)$, and let $X:=X(R, \mathfrak{F}, \Phi)$ be the associated variety. We refer to the variety $X$ as an intrinsic quadric.

Proposition 9.4. Let $(R, \mathfrak{F}, \Phi)$ and $X:=X(R, \mathfrak{F}, \Phi)$ be as above. Then the following statements hold:

(i) If $\mathcal{O}(X)=\mathbb{K}$ holds, then the defining quadric $q$ is homogeneous in the usual sense:

$$
q\left(T_{1}, \ldots, T_{r}\right)=\sum_{i \leq j} \alpha_{i j} T_{i} T_{j} .
$$

(ii) If $q$ is homogeneous with a representation as in (i), then its degree $\operatorname{deg}(q) \in$ $K$ satisfies

$$
\operatorname{deg}(Q)=w_{i}+w_{j} \quad \text { whenever } \alpha_{i j} \neq 0 .
$$

Proof. Only for (i) there is something to show. We first show that $q$ contains no linear term. Assume the contrary. Then, after suitably renumbering the indeterminates, we find $\alpha_{1} \in \mathbb{K}^{*}$ and a quadric $q^{\prime}$ not containing $T_{1}$ as a monomial such that we have

$$
q\left(T_{1}, \ldots, T_{r}\right)=\alpha_{1} T_{1}+q^{\prime}\left(T_{1}, \ldots, T_{r}\right) .
$$

We claim that $q^{\prime}$ does not even depend on $T_{1}$. Otherwise, there were a monomial $P$ in $q^{\prime}\left(T_{1}, \ldots, T_{r}\right)$ such that $P=T_{1} P^{\prime}$ holds with a non-trivial monomial $P^{\prime}$. This implies $\operatorname{deg}\left(P^{\prime}\right)=0 \in K$, which, in view of Proposition 8.2, is a contradiction to the assumption $\mathcal{O}(X)=\mathbb{K}$.

Having convinced ourselves that the quadric $q^{\prime}$ does not depend on $T_{1}$, we see that there is a well-defined isomorphism

$$
R \rightarrow \mathbb{K}\left[T_{2}, \ldots, T_{r}\right], \quad T_{1} \mapsto-q^{\prime}\left(T_{2}, \ldots, T_{r}\right), T_{2} \mapsto T_{2}, \ldots, T_{r} \mapsto T_{r} .
$$

Consequently, $R$ is again a polynomial ring. But this contradicts the assumption that the shortest systems of generators of $R$ are of length $r=\operatorname{dim}(R)+1$.

Thus, we saw that $q$ contains no non-trivial linear terms. If $q$ would contain a constant term $c \neq 0$, then $q$ would be of degree zero in $K$. This is excluded by $\mathcal{O}(X)=\mathbb{K}$ and Proposition 8.2 .

We now restrict to the case that $\mathcal{O}(X)=\mathbb{K}$ holds, and that $q$ is of full rank; we then refer to $X$ as a full intrinsic quadric. Note that factoriality of $R$ implies $r \geq 5$ for any full intrinsic quadric $X$.

Examples are provided as follows: take any quadric $q \in \mathbb{K}\left[T_{1}, \ldots, T_{r}\right]$, homogeneous in the usual sense and of full rank $r \geq 5$, any $K$-grading making $q$ and the $T_{i}$ homogeneous such that the degrees of $T_{i}$ are all nontrivial, generate a strictly convex cone, and generate $K$ as a lattice, and, finally, choose an $\mathfrak{F}$-bunch for the system $\mathcal{F}=\left\{f_{1}, \ldots, f_{r}\right\}$ of the classes $f_{i}$ of the indeterminates $T_{i}$.

Proposition 9.5. Let $(R, \mathfrak{F}, \Phi)$ be as above, suppose that the associated intrinsic quadric $X=X(R, \mathfrak{F}, \Phi)$ is full, and let $q=\sum \alpha_{i j} T_{i} T_{j}$ be the defining equation.

(i) For $\gamma_{0} \in \operatorname{rlv}(\Phi)$, the stratum $X\left(\gamma_{0}\right)$ consists of regular points if and only if $Q\left(\operatorname{lin}\left(\gamma_{0}\right) \cap E\right)=K$ holds.

(ii) A face $\gamma_{0} \preceq \gamma$ is an $\mathfrak{F}$-face if and only if the number of pairs $e_{i}, e_{j} \in \gamma_{0}$ with $\alpha_{i j} \neq 0$ differs from one. 
Proof. Since $q$ is of full rank, the origin is the only singular point of $\bar{X}=\operatorname{Spec}(R) \subset$ $\mathbb{K}^{r}$. Since $\mathcal{O}(X)=\mathbb{K}$ holds, the open set $\widehat{X} \subset \bar{X}$ defined in (2.8.1) does not contain the origin. Thus, the first assertion follows from Proposition 5.6. The second assertion is an immediate consequence of Proposition 9.3 .

Proposition 9.6. Let $X$ be a full intrinsic quadric with total coordinate ring $\mathcal{R}(X)$. Then we have

$$
\operatorname{rank}(\mathrm{Cl}(X)) \leq \operatorname{dim}(X)+3, \quad \operatorname{dim}(\mathcal{R}(X)) \leq 2 \operatorname{dim}(X)+3 .
$$

Proof. We may work in the above setup; that means that we have $X=X(R, \mathfrak{F}, \Phi)$, where $R \cong \mathbb{K}\left[T_{1}, \ldots, T_{r}\right] /\langle q\rangle$ with a homogeneous quadric $q$ of full rank, etc. Recall that, counted with multiplicities, we have precisely $r$ degrees $w_{i}=\operatorname{deg}\left(T_{i}\right) \in K$.

Using Proposition 9.4(ii) and the fact that $q$ is of rank $r$, we see that for any $w_{i}$ there is a counterpart $w_{j}$ such that $w_{i}+w_{j}=\operatorname{deg}(q)$ holds. Since the $w_{i}$ generate $K \cong \mathrm{Cl}(X)$ as a lattice, this gives

$$
\operatorname{rank}(\mathrm{Cl}(X)) \leq \frac{r}{2}+1 .
$$

Now, by Theorem 4.2 the rank of $\operatorname{Cl}(X)$ equals $\operatorname{dim}(\mathcal{R}(X))-\operatorname{dim}(X)$. Moreover, $R \cong \mathcal{R}(X)$ is of dimension $r-1$. Together, this gives the assertion.

We shall now study intrinsic quadrics having small divisor class group. In the statements, we visualize the occuring $\mathfrak{F}$-bunches according to the discussion at the end of Section 1. The case of a one-dimensional divisor class group is simple.

Proposition 9.7. Let $X$ be a full intrinsic quadric with $\mathrm{Cl}(X) \cong \mathbb{Z}$. Then $X$ arises from a bunched ring $(R, \mathfrak{F}, \Phi)$ with $\Phi$ given by

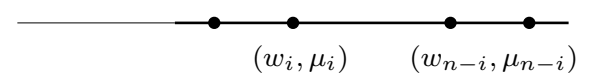

where $w_{1}, \ldots, w_{n}$ is a strictly increasing sequence of positive integers, such that $w_{i}+w_{n-i+1}=w$ holds, for some fixed $w \in \mathbb{N}$, and the weights $\mu_{i}$ satisfy

$$
\mu_{i} \geq 1, \quad \mu_{i}=\mu_{n-i}, \quad \mu_{1}+\ldots+\mu_{n} \geq 5 .
$$

The variety $X$ is always $\mathbb{Q}$-factorial, projective, and $\mathbb{Q}$-Fano, and for its dimension, we have

$$
\operatorname{dim}(X)=\mu_{1}+\ldots+\mu_{n}-2 .
$$

Note that for a full intrinsic quadric $X=X(R, \mathfrak{F}, \Phi)$ with a one-dimensional divisor class group, the minimal toric ambient variety $Z(R, \mathfrak{F}, \Phi)$ as defined in Proposition 3.7 is a weighted projective space. Moreover, $X$ is smooth if and only if all numbers $w_{i}$ equal one, and in this case it is merely a smooth projective quadric.

An explicit example of a smooth projective full intrinsic quadric with divisor class group one is the Grassmannian $G(2,4)$, studied in Examples 2.6, 2.14, and 3.4 One may also consider "weighted" versions of this Grassmannian; this provides singular varieties.

Example 9.8. Let $R:=\mathbb{K}\left[T_{1}, \ldots, T_{6}\right] /\left\langle T_{1} T_{6}-T_{2} T_{5}+T_{3} T_{4}\right\rangle$, and let $\mathfrak{F}$ consist of the classes of the $T_{i}$. Consider the $\mathfrak{F}$-bunch $\Phi$ as in Proposition 9.7 defined by the weights $w_{i}:=i$ and the multiplicities $\mu_{i}=1$, where $i=1, \ldots, 6$.

Then the corresponding variety $X:=X(R, \mathfrak{F}, \Phi)$ is not smooth, its Picard group is of index 60 in its divisor class group $\mathrm{Cl}(X)=\mathbb{Z}$, and its canonical divisor generates a sublattice of index 14 in $\mathrm{Cl}(X)$. In particular, $X$ is not Fano. 
Now we turn to the case of smooth full intrinsic quadrics with divisor class group $\mathbb{Z}^{2}$. We give the "intrinsic quadrics version" of Kleinschmidt's classification [20] of smooth complete toric varieties with Picard number two; compare also [4, Prop. 11.1].

Theorem 9.9. Let $X$ be a smooth full intrinsic quadric with $\mathrm{Cl}(X) \cong \mathbb{Z}^{2}$. Then $X$ arises from a bunched ring $(R, \mathfrak{F}, \Phi)$ with an $\mathfrak{F}$-bunch $\Phi$ given by one of the following figures:
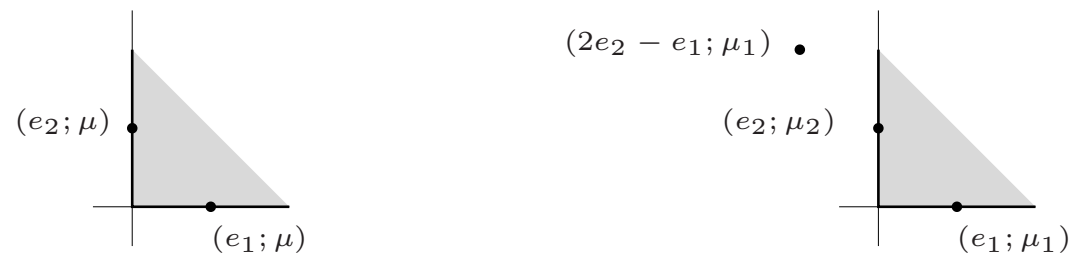

where in the left-hand side case, $\mu \geq 3$ holds, and in the right-hand side case, one has $\mu_{i} \geq 1$ and $2 \mu_{1}+\mu_{2} \geq 5$. Any such $X$ is projective, and its dimension is given by

$$
\operatorname{dim}(X)=2 \mu-3 \quad \text { or } \quad \operatorname{dim}(X)=2 \mu_{1}+\mu_{2}-3,
$$

where the first equation corresponds to the l.h.s. case, and the second one to the r.h.s. case. The variety $X$ is Fano if and only if $\Phi$ belongs to the l.h.s. case. Moreover, different figures define non-isomorphic varieties.

The proof is presented in the next section; it is an interplay of the combinatorics of bunches, and the symmetry condition 9.4(ii). For divisor class groups of higher rank and/or equations of higher degree, this type of classification problem seems to pose new and elementary but non-trivial combinatorial challenges.

\section{INTRINSIC QUADRICS II}

In this section we prove Theorem 9.9. The setup is the following: $X$ is a smooth full intrinsic quadric with divisor class group of rank two, arising from a bunched ring $(R, \mathfrak{F}, \Phi)$. According to Proposition 9.4, the defining quadric then can be written as

$$
q=\sum_{i \leq j} \alpha_{i j} T_{i} T_{j}
$$

As usual, $(E \stackrel{Q}{\longrightarrow} K, \gamma)$ is the projected cone associated to $\mathfrak{F}$, so, the lattice $K$ is of rank two. We set $w:=\operatorname{deg}(q)$, and by $e_{1}, \ldots, e_{r}$ we denote the primitive generators of $\gamma$. We call the images $Q\left(e_{i}\right)$ the weight vectors. Here comes a short list of arguments used frequently in the proof.

Lemma 10.1. In the above situation, we have the following statements:

(i) Let $e_{i}$ and $e_{j}$ be such that $2 Q\left(e_{i}\right), 2 Q\left(e_{j}\right)$ and $Q\left(e_{i}\right)+Q\left(e_{j}\right)$ are all different from $w=\operatorname{deg}(q)$. Then $\operatorname{cone}\left(e_{i}, e_{j}\right)$ is an $\mathfrak{F}$-face.

(ii) Let $e_{i}, e_{j}$ and $e_{k}$ be pairwise different such that $Q\left(e_{j}\right)$ and $Q\left(e_{i}\right)$ lie on a common ray and $2 Q\left(e_{k}\right) \neq w$ holds. Then $Q\left(e_{i}\right)$ and $Q\left(e_{k}\right)$ generate a projected $\mathfrak{F}$-face.

(iii) Let $e_{i}$ and $e_{k}$ be such that $2 Q\left(e_{i}\right)=w$ and $Q\left(e_{k}\right) \notin \mathbb{Q} \geq 0 Q\left(e_{i}\right)$ hold. Then, $Q\left(e_{i}\right)$ and $Q\left(e_{k}\right)$ generate a projected $\mathfrak{F}$-face if and only if there is a $j \neq i$ with $Q\left(e_{j}\right) \in \mathbb{Q}_{\geq 0} Q\left(e_{i}\right)$. 
(iv) For every index $i$ there exists an index $j$, not necessarily different from $i$, such that $Q\left(e_{i}\right)+Q\left(e_{j}\right)=w$ holds.

(v) If cone $\left(e_{i} ; i \in I\right)$ is a relevant $\mathfrak{F}$-face, then the images $Q\left(e_{i}\right), i \in I$, generate the lattice.

Proof. For (i), just note that in this case $\alpha_{i i}, \alpha_{i j}$ and $\alpha_{j j}$ vanish. Thus, the assertion follows from Proposition 9.5)(ii).

For (ii), note that $\alpha_{k k}=0$ holds. To define an $\mathfrak{F}$-face $\gamma_{0} \preceq \gamma$ projecting onto cone $\left(Q\left(e_{i}\right), Q\left(e_{k}\right)\right)$, we go through the possible cases and apply Proposition 9.5(ii):

$$
\begin{aligned}
& \alpha_{i i}=0, \quad \alpha_{i k}=0 \text { : } \\
& \text { 一 }, \quad \alpha_{i k} \neq 0, \quad \alpha_{i j} \neq 0 \text { : } \\
& \gamma_{0}:=\operatorname{cone}\left(e_{i}, e_{k}\right) \text {, } \\
& \text { - }, \quad-\quad, \alpha_{j k} \neq 0 \text { : } \\
& \gamma_{0}:=\operatorname{cone}\left(e_{i}, e_{j}, e_{k}\right) \text {, } \\
& -\quad, \quad- \\
& \alpha_{i j}=\alpha_{j k}=0 \\
& \alpha_{j j}=0 \text { : } \\
& \gamma_{0}:=\operatorname{cone}\left(e_{i}, e_{j}, e_{k}\right) \text {, } \\
& \alpha_{j j} \neq 0: \quad \gamma_{0}:=\operatorname{cone}\left(e_{i}, e_{j}, e_{k}\right) \text {. }
\end{aligned}
$$

By symmetry, this settles as well the case $\alpha_{j j}=0$. If both $\alpha_{i i}$ and $\alpha_{j j}$ differ from zero, then we can take $\gamma_{0}:=\operatorname{cone}\left(e_{i}, e_{j}, e_{k}\right)$.

We prove (iii). The "if" part is a direct application of (ii). To see the "only if" part, assume that only $e_{i}$ is mapped into $\mathbb{Q}_{\geq 0} Q\left(e_{i}\right)$. Then, by regularity of $q$, we must have $\alpha_{i i} \neq 0$.

Moreover, for all $j, l$ different from $i$, with $Q\left(e_{j}\right)$ and $Q\left(e_{l}\right)$ lying in the cone generated by $Q\left(e_{i}\right)$ and $Q\left(e_{k}\right)$, we have $Q\left(e_{j}\right)+Q\left(e_{l}\right) \neq w$, and hence $\alpha_{j l}=0$. Thus, Proposition 9.5(ii) tells us that there is no $\mathfrak{F}$-face $\gamma_{0} \preceq \gamma$ with $Q\left(\gamma_{0}\right)=$ $\operatorname{cone}\left(Q\left(e_{i}\right), Q\left(e_{k}\right)\right)$. Hence the latter cone is not a projected $\mathfrak{F}$-face.

We turn to (iv). Suppose that some index $i$ satisfies $Q\left(e_{i}\right)+Q\left(e_{j}\right) \neq w$ for all indices $j$. Then this implies $\alpha_{i j}=0$ for all $j$. But this contradicts the regularity of the defining quadric $q$.

Finally, assertion (v) is a direct consequence of smoothness of $X$ and Proposition 9.5 (i).

Proof of Theorem 9.9. We have to show that $\Phi$ admits a representation as in the figures shown in Theorem 9.9. First of all, as $X$ admits only constant functions, Proposition 8.2 shows that $\vartheta:=Q(\gamma)$ is strictly convex, and that all weight vectors are different from zero.

Step 1. The $\mathfrak{F}$-bunch $\Phi$ consists of a single cone $\tau$.

Assume the converse. Then we can choose two different cones $\tau_{1}, \tau_{2} \in \Phi$. Both are images of relevant faces, and thus they are two dimensional. By the defining properties of an $\mathfrak{F}$-bunch, we have $\tau_{1}^{\circ} \cap \tau_{2}^{\circ} \neq \emptyset$. Hence, $\tau_{12}:=\tau_{1} \cap \tau_{2}$ is of dimension two. Moreover, $\tau_{12} \neq \tau_{i}$ holds. Thus, there are pairwise different weight vectors $x_{1}$, $x_{2}, y_{1}$ and $y_{2}$ such that

$$
\tau_{1}=\operatorname{cone}\left(x_{1}, y_{1}\right), \quad \tau_{2}=\operatorname{cone}\left(x_{2}, y_{2}\right), \quad \tau_{12}=\operatorname{cone}\left(x_{2}, y_{1}\right) .
$$

We claim that $\tau_{12}$ is not a projected $\mathfrak{F}$-face. Indeed, for any given $\sigma \in \Phi$, we have $\sigma^{\circ} \cap \tau_{i}^{\circ} \neq \emptyset$. Using the fact that $\sigma$ and the $\tau_{i}$ are contained in the strictly convex cone $\vartheta=Q(\gamma)$, we obtain $\sigma^{\circ} \cap \tau_{12}^{\circ} \neq \emptyset$. Thus, if $\tau_{12}$ were a projected $\mathfrak{F}$-face, it would contain an element of $\Phi$. This contradicts $\tau_{12} \subsetneq \tau_{i} \in \Phi$.

Next we claim that there exist two-dimensional $\mathfrak{F}$-faces $\gamma_{i} \preceq \gamma$ with $Q\left(\gamma_{i}\right)=$ $\tau_{i}$. For this, we first exclude $2 x_{2}=w$ : otherwise, since $\tau_{2}$ is a projected $\mathfrak{F}$-face, Lemma 10.1(iii) provides a weight vector $x_{2}^{\prime} \in \mathbb{Q}_{\geq 0} x_{2}$. But then, again using 
Lemma 10.1(iii), we see that $\tau_{12}$ is a projected $\mathfrak{F}$-face. This contradicts the previous claim. Similarly, one excludes $2 y_{1}=w$.

Since $\tau_{12}$ is not a projected $\mathfrak{F}$-face, Lemma 10.1(i) gives $x_{2}+y_{1}=w$. As an immediate consequence, we obtain $x_{i}+y_{i} \neq w$ and $2 y_{2} \neq w \neq 2 x_{1}$. Hence, Lemma10.1(i) tells us that the $\tau_{i}$ are images of two-dimensional $\mathfrak{F}$-faces $\gamma_{i}$, respectively, and the claim is verified.

As remarked before, the last claim implies that the $\tau_{i}$ are regular, and that we may assume $x_{1}=(1,0)$ and $y_{1}=(0,1)$. Write $x_{2}=(a, b)$ with $a, b>0$. Then, by strict convexity of $\vartheta=Q(\gamma)$, we have $y_{2}=(-c, d)$ with $c, d>0$. The regularity of $\tau_{2}$ implies

$$
1=a d+b c \geq 2 .
$$

But this is impossible. Thus, the $\mathfrak{F}$-bunch $\Phi$ cannot contain more than one cone. This ends the proof of Step 1.

In the sequel, we denote by $\tau$ the unique cone of $\Phi$, and we denote by $x$ and $y$ the weight vectors of minimal length satisfying $\tau=\operatorname{cone}(x, y)$.

Step 2. If $v \in \tau^{\circ}$ is a weight vector, then it satisfies $2 v=w$.

Assume that $2 v \neq w$ holds. Then we can exclude $2 x=w$. If so, the fact that $\tau$ is a projected $\mathfrak{F}$-face and Lemma 10.1 (iii) provide $e_{i} \neq e_{j}$, both mapping into $\mathbb{Q}_{\geq 0} x$. Again Lemma 10.1(iii) shows that cone $(x, v)$ is a projected $\mathfrak{F}$-face. This contradicts the defining property of the $\mathfrak{F}$-bunch $\Phi=\{\tau\}$. Similarly we exclude $2 y=w$.

Since we cannot have $x+v=w$ and $y+v=w$ simultaneously, Lemma 10.1(i) shows that at least one of $\operatorname{cone}(x, v)$ and $\operatorname{cone}(v, y)$ is a projected $\mathfrak{F}$-face. As before, this contradicts the defining property of the $\mathfrak{F}$-bunch $\Phi=\{\tau\}$. Thus, we proved that any weight vector $v$ contained in $\tau^{\circ}$ must fulfill $2 v=w$.

Step 3. The cone $\tau$ is regular.

Suppose that $\tau$ is not regular. Since $\tau$ is a projected $\mathfrak{F}$-face, the lattice $K$ is generated by certain weight vectors lying in $\tau$. By non-regularity, at least one of these weight vectors, say $v$, has to lie in $\tau^{\circ}$. By the previous step, there is only one possibility: $v$ is determined by $2 v=w$.

Consider any pair $x^{\prime}, y^{\prime}$ of weight vectors generating the cone $\tau$. Then $2 x^{\prime} \neq w$ and $2 y^{\prime} \neq w$ hold. Using this, we can conclude $x^{\prime}+y^{\prime}=w$, because otherwise, Lemma 10.1(i) and (v) would show that $\tau$ is regular. This implies that $x, y$, and $v$ are the only weight vectors in $\tau$.

Consequently, $x, y, v$ form a system of generators of $K$ satisfying $x+y=2 v$. But then $x, v$, as well as $v, y$, are bases. Thus, we may prescribe $x, v$ and then determine $w$ and $y$ as follows:

$$
x=(1,0), \quad v=(1,1), \quad w=(2,2), \quad y=(1,2) .
$$

Now, suppose that there exists a weight vector $u$ different from $x, y$ and $v$. Then, because of Lemma 10.1(iv) and $w=2 v$, there must exist a weight vector $u^{\prime} \neq u$ with $u+u^{\prime}=w$. Since $u$ as well as $u^{\prime}$ does not belong to $\tau$, and $u+u^{\prime} \in \tau^{\circ}$ holds, we have $\tau \subset$ cone $\left(u, u^{\prime}\right)$. By possibly interchanging $u$ and $u^{\prime}$, we achieve that cone $(x, u)$ contains $\tau$.

Lemma 10.1(i) and (v) say that $x$ and $u$ generate the lattice. Consequently, $u=(a, 1)$ for some integer $a$. Note that $a \leq 0$ holds, because of $u \notin \tau$. But this 
leads to a contradiction:

$$
u^{\prime}=w-u=(2-a, 1) \in \tau .
$$

So, we arrived at the following picture: the only weight vectors are $x, v$ and $y$, and we have $\tau=\operatorname{cone}(x, y)$. The multiplicities must satisfy $\mu(x)=\mu(y)$ and $\mu(v)=1$. The latter is, once more, a consequence of Lemma 10.1(iii): a higher multiplicity $\mu(v)$ would imply that $\operatorname{cone}(x, v)$ and $\operatorname{cone}(v, y)$ are projected $\mathfrak{F}$-faces, which contradicts $\Phi=\{\tau\}$.

Since $q$ has at least 5 variables, we see that $\mu(x)=\mu(y) \geq 2$ holds. Having in mind that $q$ is a regular quadric, this implies that there are at least two different pairs $i, j$ and $i^{\prime}, j^{\prime}$ with $Q\left(e_{i}\right)=Q\left(e_{i^{\prime}}\right)=x$ and $Q\left(e_{j}\right)=Q\left(e_{j^{\prime}}\right)=y$ such that $\alpha_{i j} \neq 0 \neq \alpha_{i^{\prime} j^{\prime}}$ holds. Thus, Proposition 9.5 and Lemma 10.1(v) tell us that $x$ and $y$ generate the lattice. A contradiction.

Step 4 . The weight vectors $x$ and $y$ form a lattice basis.

First consider the case that there is a weight vector $v \in \tau^{\circ}$. Then we know by Step 2 that $2 v=w$ holds. Consider any pair $x^{\prime}, y^{\prime}$ of weight vectors generating $\tau$ as a convex cone. If we have $x^{\prime}+y^{\prime} \neq w$, then Lemma 10.1(i) and (v) shows that $x^{\prime}$ and $y^{\prime}$ generate the lattice, hence $x^{\prime}=x$ and $y^{\prime}=y$, and we are done.

So, we may assume $x^{\prime}+y^{\prime}=w$. But this fixes $x^{\prime}$ and $y^{\prime}$. Thus, we again have that $x$ and $y$ are the only weight vectors lying in the boundary of $\tau$. Lemma 10.1(v) says that $x, y$ and $v$ generate the lattice.

Because of $x+y=2 v$, the pair $x, v$ is a basis. Thus, we may assume that $x=(1,0)$ and $v=(1,1)$ holds. This implies

$$
y=w-x=2 v-x=(1,2) .
$$

So, we arrived at a contradiction to regularity of $\tau$. That means $x+y=w$ cannot happen, and the case that there exists a weight vector $v \in \tau^{\circ}$ is settled.

Let us consider the case that all weight vectors of $\tau$ lie in the boundary. If $x$ and $y$ are the only ones, then we succeed with Lemma 10.1(v). So, suppose that on some ray, there are at least two different weight vectors, say $y^{\prime}, y^{\prime \prime} \in \mathbb{Q}_{\geq 0} y$.

Then, if $2 y^{\prime}=w$, then Lemma 10.1(i) and (v) show that $x$ and $y^{\prime \prime}$ generate the lattice. This implies $y^{\prime \prime}=y$, and we are done. Similarly, we settle the case $2 y^{\prime \prime}=w$. Next, if $2 x, 2 y^{\prime}$ and $2 y^{\prime \prime}$ all differ from $w$, then Lemma 10.1(i) and (v) show that either the pair $x, y^{\prime}$ or the pair $x, y^{\prime \prime}$ is a lattice basis.

Finally, suppose that $2 x=w$ holds. Then, by a suitable choice of coordinates, we may achieve that

$$
x=(a, 0), \quad w=(2 a, 0), \quad y^{\prime}=\left(0, b^{\prime}\right), \quad y^{\prime \prime}=\left(0, b^{\prime \prime}\right)
$$

hold with positive integers $a$, and $b^{\prime} \neq b^{\prime \prime}$. According to Lemma 10.1(iv), we have weight vectors

$$
z^{\prime}=w-y^{\prime}=\left(2 a,-b^{\prime}\right), \quad z^{\prime \prime}=w-y^{\prime \prime}=\left(2 a,-b^{\prime \prime}\right) .
$$

By Lemma 10.1(i) and (v), the vectors $y^{\prime}$ and $z^{\prime \prime}$ generate the lattice. This implies $2 a b^{\prime}= \pm 1$, which is impossible.

So, having passed Step 4, we know that $x$ and $y$ form a lattice basis. According to Lemma 10.1(iv), there exist weight vectors $x^{\prime}$ and $y^{\prime}$ with $x+x^{\prime}=y+y^{\prime}=w$.

Step 5 . The case $x^{\prime} \neq x$ and $y \neq y^{\prime}$. 
We shall show that the present situation amounts to the left-hand side figure of Theorem 9.9. Note that we have

$$
2 x \neq w, \quad 2 x^{\prime} \neq w, \quad 2 y \neq w, \quad 2 y^{\prime} \neq w, \quad x+y^{\prime} \neq w, \quad x^{\prime}+y \neq w .
$$

In particular, using Step 2, we can conclude from this list that neither $x^{\prime}$ nor $y^{\prime}$ belong to $\tau^{\circ}$.

We claim that $\tau \subset \operatorname{cone}\left(x^{\prime}, y^{\prime}\right)$ holds. For the verification of this claim, we may assume that $x=(1,0)$ and $y=(0,1)$ hold. Since all weight vectors belong to the strictly convex cone $\vartheta=Q(\gamma)$, we see that $\tau \not \subset$ cone $\left(x^{\prime}, y^{\prime}\right)$ can only happen if $x^{\prime}$ and $y^{\prime}$ both lie either in the upper or the right half plane.

By symmetry, it suffices to treat the case that $x^{\prime}$ and $y^{\prime}$ both lie in the upper half plane. There are integers $a$ and $b$ such that

$$
x^{\prime}=(a, b), \quad w=x+x^{\prime}=(a+1, b), \quad y^{\prime}=w-y=(a+1, b-1) .
$$

Note that $b \geq 1$ holds because $y^{\prime}$ belongs to the upper half plane. Moreover, we have $a \leq 0$ because $x^{\prime}$ does not belong to $\tau^{\circ}$.

We settle the case $b=1$. Strict convexity of $\vartheta$ and $x, y^{\prime} \in \vartheta$ imply $a \geq-1$. If $a=0$, then $x^{\prime}=y$ and $y^{\prime}=x$ holds, and we are done. If $a=-1$ holds, then we obtain $y^{\prime}=0$, which contradicts the characterization 8.2 of $\mathcal{O}(X)=\mathbb{K}$. So we may assume $b \geq 2$ from now on.

Since $2 x, 2 y^{\prime}$ and $x+y^{\prime}$ all differ from $w$, Lemma 10.1(i) yields that cone $\left(x, y^{\prime}\right)$ is the image of a two-dimensional $\mathfrak{F}$-face. By the defining properties of $\Phi$, we can conclude that cone $\left(x, y^{\prime}\right)$ contains $\tau$. This means $a \leq-1$. Moreover, by Lemma $10.1(\mathrm{v})$, the vectors $x$ and $y^{\prime}$ generate the lattice. In particular, $b=2$ holds. Summing up, we obtained

$$
x^{\prime}=(a, 2), \quad w=(a+1,2), \quad y^{\prime}=(a+1,1), \quad a \leq-1 .
$$

In view of Step 2, this excludes in particular, the existence of weight vectors inside $\tau^{\circ}$. We can even exclude existence of weight vectors different from $x$ and $y$ in the whole right half plane: such a vector is of the form $u=(\zeta, \eta)$ with $\zeta \geq 0$ and $\eta \leq 0$. By Lemma 10.1(iv), we have a further weight vector

$$
u^{\prime}=w-u=(a+1-\zeta, 2-\eta) .
$$

Since $2 x, 2 u^{\prime}$ and $x+u^{\prime}$ all differ from $w$, we may apply Lemma 10.1(i) and (v), and see that $x$ and $u^{\prime}$ generate the lattice $K$. This contradics that fact that the second coordinate of $u^{\prime}$ is at least two. So, there are no weight vectors different from $x$ in the fourth quadrant.

Using the second property of Definition 2.1(ii), we obtain that there are at least two different $e_{i}, e_{j} \in \gamma$ mapping into the ray through $x$. Thus, applying Lemma 10.1(ii) to $e_{i}, e_{j}$ and any $e_{k}$ mapping to $x^{\prime}$ shows that $x^{\prime}$ and the images of $e_{i}$ and $e_{j}$ generate the lattice, which is a contradiction.

This ends the verification of the first claim of Step 5, and we know now that $\tau \subset \operatorname{cone}\left(x^{\prime}, y^{\prime}\right)$ holds.

Next we claim that $x^{\prime}$ and $y^{\prime}$ form a lattice basis. Indeed, if $x^{\prime}+y^{\prime}=w$ holds, then we have $x^{\prime}=y$ and $y^{\prime}=x$, and we are done. If $x^{\prime}+y^{\prime} \neq w$ holds, then Lemma 10.1(i) and (v) tell us that $x^{\prime}$ and $y^{\prime}$ form a lattice basis, and the claim is verified.

By a suitable change of coordinates, we achieve that $x^{\prime}=(0,1)$ and $y^{\prime}=(1,0)$ hold. Write $x=(k, l-1)$. Then $y=(k-1, l)$ holds with $k, l>0$. The fact that $x$ 
and $y$ form a lattice basis translates to

$$
\pm 1=k l-(k-1)(l-1)=k+l-1 .
$$

Since $k, l$ are positive integers, we eventually obtain $k=l=1$ and therefore $x=y^{\prime}$ and $x^{\prime}=y$. Thus, we have $w=(1,1)$. According to Step 2, this shows, in particular, that $\tau^{\circ}$ contains no weight vectors.

In order to arrive at the left-hand side figure of Theorem 9.9, we still have to show that $x$ and $y$ are the only weight vectors.

Suppose that there is a weight vector $u$, which differs from $x$ and from $y$. By Lemma 10.1(iv), we then find a weight vector $u^{\prime}$ with $u+u^{\prime}=w=(1,1)$. Note that neither $u$ nor $u^{\prime}$ belong to $\tau$. Moreover, they lie in different quadrants, because of $u+u^{\prime}=w \in \tau^{\circ}$.

By possibly interchanging $u$ and $u^{\prime}$, we achieve that $u=(a,-b)$ holds with positive integers $a$ and $b$. Now, Lemma 10.1(i) applies to cone $(u, y)$. It shows that $u$ and $y$ form a lattice basis. Thus, we obtain $a=1$. This leads to a contradiction:

$$
u^{\prime}=w-u=(0,1+b) \in \tau .
$$

Step 6. The case $x=x^{\prime}$ or $y=y^{\prime}$.

We treat as an example the case $y=y^{\prime}$. Note that then $2 y=w$ holds. Thus, according to Step 2, there are no weight vectors in $\tau^{\circ}$. By a suitable choice of coordinates we achieve

$$
x=(1,0), \quad y=(0,1), \quad w=(0,2), \quad x^{\prime}=(-1,2) .
$$

We show that we are in the situation of the right-hand side figure. That means that we have to exclude existence of further weight vectors. Assume to the contrary that there is a weight vector $u$, which is different from $x, x^{\prime}$ and $y$.

Lemma 10.1(iv) provides a counterpart $u^{\prime}$ satisfying $u+u^{\prime}=w$. Since all weight vectors lie in the strictly convex cone $\vartheta=Q(\gamma)$, neither $u$ nor $u^{\prime}$ can belong to the negative quadrant. Consequently, after possibly interchanging $u$ and $u^{\prime}$, there are non-negative integers $a$ and $b$ with

$$
u=(a,-b), \quad u^{\prime}=w-u=(-a, 2+b) .
$$

Since $2 x, 2 u^{\prime}$ and $x+u^{\prime}$ differ from $w$, Lemma 10.1(i) and (v) show that $u^{\prime}$ and $x$ generate the lattice. This contradicts the explicit presentation of $u^{\prime}$. Thus, Step 6 is done.

So, we proved that the $\mathfrak{F}$-bunches corresponding to the smooth intrinsic quadrics $X$ with $\mathcal{O}(X)=\mathbb{K}$ arise from one of the figures listed in the assertion. Quasiprojectivity and the statements on being Fano are due to our general results. Moreover, Proposition 8.3 gives completeness of the varieties in question.

In order to see that different figures belong to non-isomorphic varieties, note first that the left- and the right-hand side have nothing to do with each other, because one is Fano and the other is not. Moreover, different choices of the weights $\mu$ on the left-hand side give rise to varieties of different dimension.

The right-hand side is a little more subtle. First note that the occuring weight vectors form a Hilbert basis for the cone in $K=\mathrm{Cl}(X)$ generated by the degrees that admit non-trivial homogeneous elements in $R=\mathcal{R}(X)$. This Hilbert basis as well as the dimensions of the corresponding spaces of homogeneous sections are invariants of $X$. But the latter determine $\mu_{1}$ and $\mu_{2}$. 


\section{Surfaces}

In this section we focus on the case of surfaces. We first present some general results, and then turn to explicit examples. The first observation is that, in our setup, complete surfaces are determined by their total coordinate ring.

Proposition 11.1. Let $X_{1}$ and $X_{2}$ be normal $A_{2}$-maximal surfaces with finitely generated free divisor class groups and finitely generated total coordinate rings $R_{1}$ and $R_{2}$ respectively, and suppose that $X_{2}$ is complete. Then we have $X_{1} \cong X_{2}$ if and only if $R_{1} \cong R_{2}$ as graded rings.

Proof. Only for the "if" part there is something to show. By Theorem 4.2, we may assume that $X_{2}$ arises from a bunched ring $\left(R_{2}, \mathfrak{F}_{2}, \Phi_{2}\right)$. Let $\alpha: R_{2} \rightarrow R_{1}$ be a graded isomorphism, and set $\mathfrak{F}_{1}:=\alpha\left(\mathfrak{F}_{2}\right)$. Then, by Assertion 4.2), we may assume $X_{1}=X\left(R_{1}, \mathfrak{F}_{1}, \Phi_{1}\right)$ with an $\mathfrak{F}_{1}$-bunch $\Phi_{1}$.

Now, look at $\bar{X}_{i}:=\operatorname{Spec}\left(R_{i}\right)$, and the isomorphism $\bar{\varphi}: \bar{X}_{1} \rightarrow \bar{X}_{2}$ defined by $\alpha: R_{2} \rightarrow R_{1}$. Moreover, consider the open subsets $\widehat{X}_{i} \subset \bar{X}_{i}$, the quotient presentations $p_{i}: \widehat{X}_{i} \rightarrow X_{i}$ of Proposition [2.9] and the open sets $W_{i} \subset X_{i}$ as studied in Lemma 3.5

Then $\bar{\varphi}$ maps $p_{1}^{-1}\left(W_{1}\right)$ onto $p_{2}^{-1}\left(W_{2}\right)$, and thus induces an isomorphism $W_{1} \rightarrow$ $W_{2}$. Since the surface $X_{2}$ is complete, and the complements $X_{i} \backslash W_{i}$ are of codimension at least two and hence at most finite, the map $W_{1} \rightarrow W_{2}$ extends to an open embedding $X_{1} \rightarrow X_{2}$. Since $X_{1}$ is $A_{2}$-maximal, $X_{1} \rightarrow X_{2}$ is onto.

As an application of the independence of a complete surface $X(R, \mathfrak{F}, \Phi)$ from the particular choice of $\mathfrak{F}$ and $\Phi$, we obtain a general projectivity criterion.

Proposition 11.2. Let $X$ be a normal $A_{2}$-maximal surface with finitely generated free divisor class group and finitely generated total coordinate ring. If $\mathcal{O}(X)=\mathbb{K}$ holds, then $X$ is projective.

Proof. By Theorem4.2 we may assume that $X=X(R, \mathfrak{F}, \Phi)$ holds with a bunched ring $(R, \mathfrak{F}, \Phi)$. As in Lemma 3.1. we extend $\Phi$ to a bunch $\Theta$ in the projected cone $(E \stackrel{Q}{\longrightarrow} K, \gamma)$ associated to $\mathfrak{F}$. Consider the dual projected cone $(F \stackrel{P}{\longrightarrow} N, \delta)$, the maximal projectable fan $\widehat{\Delta}$ in $F$ correponding to $\Theta$, and its quotient fan $\Delta$ in $N$; see [4, Sec. 4].

According to Proposition 8.2 the rays of $\Delta$ generate the vector space $N_{\mathbb{Q}}$ as a cone. Consequently, Lemma 1.6 provides a polytopal fan $\Delta^{\prime}$ in $N$ having the same rays as $\Delta$. The fan $\widehat{\Delta}^{\prime}$ in $F$ generated by those faces of $\delta$ that map onto a cone of $\Delta^{\prime}$ is maximal projectable, and hence $\widehat{\Delta}^{\prime}$ corresponds to a bunch $\Theta^{\prime}$ in $(E \stackrel{Q}{\longrightarrow} K, \gamma)$; see again [4, Sec. 4].

Picking out the $\mathfrak{F}$-faces from $\operatorname{rlv}\left(\Theta^{\prime}\right)$, and taking the minimal cones among their images under $Q$, we arrive at an $\mathfrak{F}$-bunch $\Phi^{\prime}$, and hence a bunched $\operatorname{ring}\left(R, \mathfrak{F}, \Phi^{\prime}\right)$. The associated variety $X^{\prime}$ is by construction embedded into $Z_{\Theta^{\prime}}$, and hence is projective. Proposition 11.1 gives $X \cong X^{\prime}$.

We give some applications of this proposition. Goodman showed in 12 that a normal complete surface is projective, if and only if the set of its non-factorial singularities admits a common affine neighbourhood. For surfaces with a finitely generated total coordinate ring this can be sharpened. 
Corollary 11.3. A normal complete surface with finitely generated free divisor class group and finitely generated total coordinate ring is projective if and only if any two of its non-factorial singularities admit a common affine neighbourhood.

Proof. The "if" part is the interesting one. We have to show that the surface, call it $X$, is then $A_{2}$. Let $x, x^{\prime} \in X$. If both points are non-factorial, then they have a common affine neighbourhood by assumption. So, we may assume that $x$ is factorial.

Let $X^{\prime} \rightarrow X$ resolve all the non-factorial singularities different from $x^{\prime}$. Then $X^{\prime}$ is projective, hence $x$ and $x^{\prime}$ have a common affine neighbourhood $U^{\prime} \subset X^{\prime}$. After suitably shrinking $U^{\prime}$, we achieve that it does not meet exceptional curves, and hence maps onto an affine neighbourhood $U$ of $x$ and $x^{\prime}$ in $X$.

Corollary 11.4. If $X$ is a full intrinsic quadric of dimension two, then $X$ is projective and $\mathrm{Cl}(X)$ is of rank at most 5 .

Proof. The estimate for the rank of $\mathrm{Cl}(X)$ is a direct consequence of Proposition 9.6. and projectivity of $X$ is due to Proposition 11.2.

We now turn to explicit examples. The first one is a quotient of an open subset of the Grassmannian $G(2,4)$ by the action of a two-dimensional torus. Consider the ring

$$
R:=\mathbb{K}\left[T_{1}, \ldots, T_{6}\right] /\left\langle T_{1} T_{6}-T_{2} T_{5}+T_{3} T_{4}\right\rangle,
$$

and define a $\mathbb{Z}^{3}$-grading on $R$ by setting $\operatorname{deg}\left(T_{i}\right):=w_{i}$, where the vectors $w_{i} \in \mathbb{Z}^{3}$ are the following:

$$
\begin{aligned}
& w_{1}:=(1,0,1), \quad w_{2}:=(1,1,1), \quad w_{3}:=(0,1,1), \\
& w_{4}:=(0,-1,1), \quad w_{5}:=(-1,-1,1), \quad w_{6}:=(-1,0,1) .
\end{aligned}
$$

Note that in this setup, condition 9.4(ii) is fulfilled. By Proposition 11.1, these data already uniquely determine a surface, and the results of the paper give insight to the geometry.

Theorem 11.5. Let $X$ be an $A_{2}$-maximal normal variety having the $\mathbb{Z}^{3}$-graded ring $R$ as its total coordinate ring.

(i) The variety $X$ is a $\mathbb{Q}$-factorial, projective surface. It has precisely five singular points.

(ii) The Picard group $\operatorname{Pic}(X)$ is a sublattice of index 72 in the divisor class group $\mathrm{Cl}(X) \cong \mathbb{Z}^{3}$.

(iii) The semiample cone $\operatorname{Sample}(X)$ equals the moving cone $\operatorname{Mov}(X)$; it is of full dimension in $\mathrm{Cl}_{\mathbb{Q}}(X)$, and has six extremal rays.

(iv) The canonical divisor of $X$ is of the form $4 D$ with a Weil divisor $D$. The lowest locally principal multiple is $12 D$, and this is an ample divisor.

Proof. We have to represent $X$ as the variety arising from a bunched ring $(R, \mathfrak{F}, \Phi)$. As usual, let $\mathfrak{F}$ consist of the classes of the $T_{i}$ in $R$. Let $(E \stackrel{Q}{\longrightarrow} K, \gamma)$ be the associated projected cone, i.e., we have $K=\mathbb{Z}^{3}$ and $E=\mathbb{Z}^{6}$, the map $Q$ sends the $i$-th canonical base vector $e_{i}$ to $w_{i}$, and $\gamma=\mathbb{Q}_{>0}^{6}$ is the positive orthant.

According to Assertion 4.2 ) and Proposition 11.1, we may take any $\mathfrak{F}$-bunch $\Phi$. Write $\tau\left(i_{1}, \ldots, i_{r}\right)$ for the cone in $K_{\mathbb{Q}}$ generated by the vectors $w_{i_{1}}, \ldots, w_{i_{r}}$, and consider the cones

$$
\tau(1,3,5), \quad \tau(2,4,6), \quad \tau(1,6,2,5), \quad \tau(1,6,3,4), \quad \tau(2,5,3,4) .
$$


As in Proposition 9.5(ii) we see that these cones form an $\mathfrak{F}$-bunch $\Phi$. Indicating the two simplicial cones by dashed lines, we obtain the following picture:

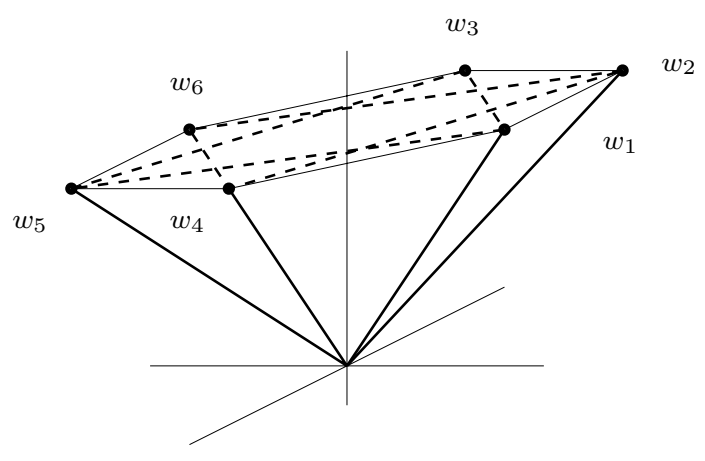

Similarly as for the projected faces, we denote the faces of $\gamma$ by $\gamma\left(i_{1}, \ldots, i_{r}\right)$. Then the collection $\operatorname{rlv}(\Phi)$ of relevant faces consists of $\gamma$ itself, all the facets of $\gamma$, and the faces

$$
\gamma(1,3,5), \quad \gamma(2,4,6), \quad \gamma(1,6,2,5), \quad \gamma(1,6,3,4), \quad \gamma(2,5,3,4) .
$$

The images of the relevant cones are of full dimension, hence $X$ is $\mathbb{Q}$-factorial. Moreover, all strata associated to the faces of dimension five or more consist of smooth points, whereas the strata associated to the faces listed in (11.5.1) are (isolated) singularities. This proves (i).

In order to prove the second assertion, recall from Proposition 7.1 that the Picard group of $X$ is given by

$$
\operatorname{Pic}(X)=\bigcap_{\gamma_{0} \in \operatorname{cov}(\Phi)} Q\left(\operatorname{lin}\left(\gamma_{0}\right) \cap E\right) .
$$

Since the images of the faces of dimension five or six generate $K$, we may restrict the intersection to the faces listed in (11.5.1). Thus, we obtain that $\operatorname{Pic}(X)$ is generated as a sublattice of $K$ by the vectors

$$
(2,4,0), \quad(0,6,0), \quad(0,0,6) .
$$

We turn to (iii). Theorem 7.3 gives us the semiample cone of $X$. Namely, we have to intersect the members of $\Phi$. Explicitly, this means that $\operatorname{Sample}(X)$ has the primitive generators

$$
(1,-1,3), \quad(-1,-2,3), \quad(-1,1,3), \quad(-2,-1,3), \quad(2,1,3), \quad(1,2,3) .
$$

According to Proposition 8.5, the canonical divisor class of $X$ is represented by the vector $(0,0,-4)$. In particular, $X$ is a $\mathbb{Q}$-Fano variety. More precisely, from our description of the Picard group of $X$, we see that $(0,0,-12)$ is the least multiple, which is Cartier.

Finally, we consider a surface studied by Hassett and Tschinkel in [16, namely the minimal resolution $X$ of the $E_{6}$ cubic surface

$$
X^{\prime}:=\left\{\left[z_{0}, \ldots, z_{3}\right] \in \mathbb{P}^{3} ; z_{1} z_{2}^{2}+z_{2} z_{0}^{2}+z_{3}^{3}=0\right\} \subset \mathbb{P}^{3} .
$$

As shown in [16], the surface $X$ has a free divisor class group $\mathrm{Cl}(X)$ of rank 7 with a (quite) canonical basis

$$
F_{1}, F_{2}, F_{3}, F_{4}, F_{5}, F_{6}, \ell
$$


where the $F_{i}$ are the exceptional curves of the resolution $X \rightarrow X^{\prime}$ and $\ell$ is the strict transform of a certain distinguished line on $X^{\prime}$; see [16, Prop. 4.4].

Moreover, Hassett and Tschinkel determined the total coordinate ring of $X$. As a ring, it is given by

$$
\mathcal{R}(X)=\mathbb{C}\left[T_{1}, \ldots, T_{10}\right] /\left\langle T_{10} T_{7}^{3} T_{4}^{2} T_{5}+T_{9}^{2} T_{2}+T_{8}^{3} T_{1}^{2} T_{3}\right\rangle .
$$

The $\mathrm{Cl}(X)$-grading of $\mathcal{R}(X)$ is defined by sending $T_{i}$ to the vector in $\mathrm{Cl}(X)$ having as its coordinates with respect to the above canonical basis the $i$-th column of the following matrix:

$$
\left[\begin{array}{llllllllll}
1 & 0 & 0 & 0 & 0 & 0 & 0 & 0 & 1 & 2 \\
0 & 1 & 0 & 0 & 0 & 0 & 0 & 1 & 1 & 3 \\
0 & 0 & 1 & 0 & 0 & 0 & 0 & 1 & 2 & 4 \\
0 & 0 & 0 & 1 & 0 & 0 & 0 & 2 & 3 & 4 \\
0 & 0 & 0 & 0 & 1 & 0 & 0 & 2 & 3 & 5 \\
0 & 0 & 0 & 0 & 0 & 1 & 0 & 2 & 3 & 6 \\
0 & 0 & 0 & 0 & 0 & 0 & 1 & 2 & 3 & 3
\end{array}\right]
$$

Using Remark 9.2 it is easy to see that the classes of the $T_{i}$ in $\mathcal{R}(X)$ are indeed pairwise non-associated primes.

In order to apply our results, we have to describe $X$ as the variety arising from a bunched ring $(R, \mathfrak{F}, \Phi)$, where $R:=\mathcal{R}(X)$ with the grading lattice $K=\mathbb{Z}^{7}$. Let $\mathfrak{F}$ consist of the classes of the variables $T_{i}$ in $R$.

The associated projected cone $(E \stackrel{Q}{\longrightarrow} K, \gamma)$ is given by $E:=\mathbb{Z}^{10}$, the map $Q: E \rightarrow K$ determined by the matrix (11.5.2), and $K=\mathbb{Z}^{7}$. Similarly as above, we write $\tau\left(i_{1}, \ldots, i_{s}\right)$ for the cone in $K_{\mathbb{Q}}$ generated by $Q\left(e_{i_{1}}\right), \ldots, Q\left(e_{i_{s}}\right)$. Consider

$$
\begin{array}{ll}
\tau(1,2,3,4,7,8,9,10), & \tau(1,2,3,5,6,8,9,10), \\
\tau(1,2,3,6,7,8,9,10), & \tau(1,2,4,5,7,8,9,10), \\
\tau(2,4,5,6,7,8,9,10), & \tau(1,3,4,5,7,8,9,10) .
\end{array}
$$

Using Proposition 0.3, one sees that these are the cones of an $\mathfrak{F}$-bunch $\Phi$. Thus, Theorem 7.3 and Proposition 8.5 give us the semiample and the ample cone of $X$ and its canonical divisor class; compare [16, Prop. 4.5].

Proposition 11.6. The semiample cone Sample $(X)$ is the regular cone in $\mathbb{Z}^{7}$ having as its primitive generators the columns of the matrix:

$$
\left[\begin{array}{lllllll}
0 & 2 & 1 & 2 & 2 & 2 & 1 \\
1 & 3 & 1 & 3 & 3 & 3 & 2 \\
1 & 4 & 2 & 4 & 4 & 4 & 2 \\
2 & 4 & 3 & 4 & 5 & 6 & 4 \\
2 & 5 & 3 & 5 & 5 & 6 & 4 \\
2 & 6 & 3 & 6 & 6 & 6 & 4 \\
2 & 3 & 3 & 4 & 5 & 6 & 4
\end{array}\right]
$$

Moreover, Sample $(X)$ equals the moving cone, the ample cone is the relative interior of $\operatorname{Sample}(X)$, and the canonical divisor class of $X$ is given by the vector $-(2,3,4,4,5,6,3)$.

Finally, we provide the fan describing the minimal toric ambient variety $Z:=$ $Z(R, \mathfrak{F}, \Phi)$ of $X$ as discussed in Proposition 3.7 . Note that in the present example, $Z$ is not complete, moreover it is not even $A_{2}$-maximal. 
Proposition 11.7. The fan corresponding to the minimal toric ambient variety $Z=Z(R, \mathfrak{F}, \Phi)$ of $X$ has ten maximal cones; one of them is the negative orthant cone $((-1,0,0),(0,-1,0),(0,0,-1))$, and the remaining nine are of dimension two:

$$
\begin{array}{lll}
\text { cone }((2,3,3),(0,0,-1)), & \operatorname{cone}((1,1,3),(0,-1,0)), & \operatorname{cone}((0,1,2),(-1,0,0)), \\
\operatorname{cone}((2,3,4),(2,3,3)), & \operatorname{cone}((2,3,5),(2,3,6)), & \operatorname{cone}((1,2,4),(2,3,6)), \\
\text { cone }((1,1,3),(2,3,6)), & \operatorname{cone}((2,3,4),(2,3,5)), & \operatorname{cone}((0,1,2),(1,2,4)) .
\end{array}
$$

Proof. First we have to determine the covering collection of the $\mathfrak{F}$-bunch $\Phi$. It consists of the following ten faces of $\gamma=\operatorname{cone}\left(e_{1}, \ldots, e_{10}\right)$ :

$$
\begin{array}{lll}
\gamma(1,2,3,4,7,8,9,10), & \gamma(1,2,3,5,6,8,9,10), & \gamma(1,2,3,6,7,8,9,10), \\
\gamma(1,2,4,5,7,8,9,10), & \gamma(2,4,5,6,7,8,9,10), & \gamma(1,3,4,5,7,8,9,10), \\
\gamma(1,2,3,4,5,6,8,9), & \gamma(1,3,4,5,6,7,8,10), & \gamma(2,3,4,5,6,7,9,10), \\
\gamma(1,2,3,4,5,6,7), & &
\end{array}
$$

where, as usual, we denote cone $\left(e_{i_{1}}, \ldots, e_{i_{r}}\right)$ by $\gamma\left(i_{1}, \ldots, i_{r}\right)$. Then, the maximal cones of the fan of $Z$ are the images $P\left(\gamma_{0}^{*}\right)$, where $\gamma_{0} \in \operatorname{cov}(\Theta)$ and $P$ is the map dual to the inclusion of $M:=\operatorname{ker}(Q)$ in $E$.

\section{REFERENCES}

[1] V. Batyrev, O. Popov: The Cox ring of a del Pezzo surface. Arithmetic of higher-dimensional algebraic varieties (Palo Alto, CA, 2002), 85-103, Progr. Math. 226, Birkhäuser Boston, Boston, MA (2004). MR2029863 (2005h:14091)

[2] F. Berchtold: Lifting of morphisms to quotient presentations. Manuscripta Math. 110, 33-44 (2003). MR:1951798 (2003m:14083)

[3] F. Berchtold, J. Hausen: Homogeneous coordinates for algebraic varieties. J. Algebra 266, 636-670 (2003). MR1995130 (2004e:14004)

[4] F. Berchtold, J. Hausen: Bunches of cones in the divisor class group - A new combinatorial language for toric varieties. Int. Math. Res. Not., 261-302 (2004). MR2041065(2004m:14111)

[5] A. Białynicki-Birula, J. Święcicka: Complete quotients by algebraic torus actions. In: Group actions and vector fields. Springer LNM 956, 10-22 (1981). MR0704983 (85d:14065)

[6] A. Białynicky-Birula, J. Święcicka: Three theorems on existence of good quotients. Math. Ann. 307, 143-149 (1997). MR1427680(97j:14054)

[7] J. Boutot: Singularités rationelles et quotients par les groupes réductifs. Invent. Math. 88, 65-68 (1987). MR0877006 (88a:14005)

[8] D. Cox: The homogeneous coordinate ring of a toric variety. J. Alg. Geom. 4, 17-50 (1995). MR 1299003 (95i:14046)

[9] J. Elizondo, K. Kurano, K. Watanabe: The total coordinate ring of a normal projective variety. J. Algebra 276, 625-637 (2004). MR2058459 (2005b:14013)

[10] G. Ewald: Polygons with Hidden Vertices. Contributions to Algebra and Geometry 42, 439442 (2001). MR 1865531 (2002g:52010)

[11] W. Fulton: Introduction to toric varieties. Annals of Mathematics Studies. 131. Princeton University Press, Princeton, NJ (1993). MR1234037 (94g:14028)

[12] J.E. Goodman: Affine open subsets of algebraic varieties and ample divisors. Ann. of Math. 89, 160-183 (1969). MR0242843 (39:4170)

[13] R. Hartshorne: Algebraic geometry. Corr. 3rd printing. Graduate Texts in Mathematics, 52. Springer-Verlag, New York, Heidelberg, Berlin (1983). MR 0463157 (57:3116)

[14] J. Hausen: Producing good quotients by embedding into toric varieties. In: L. Bonavero, M. Brion (Eds): Geometry of toric Varieties. Sém. et Congr. 6, 193-212 (2002). MR2075611 (2005h:14113)

[15] M. Hochster, J. Roberts: Rings of invariants of reductive groups acting on regular rings are Cohen-Macaulay. Adv. Math. 13, 115-175 (1974). MR0347810 (50:311)

[16] B. Hassett, Y. Tschinkel: Universal torsors and Cox rings. Arithmetic of higher-dimensional algebraic varieties (Palo Alto, CA, 2002), 149-173, Progr. Math. 226., Birkhäuser Boston, Boston, MA (2004). MR2029868 (2005a:14049) 
[17] Y. Hu, S. Keel: Mori dream spaces and GIT. Michigan Math. J. 48, 331-348 (2000). MR:1786494 (2001i:14059)

[18] T. Kambayashi, P. Russell: On linearizing algebraic torus actions. J. Pure Appl. Algebra 23, 243-250 (1982). MR0644276 (83d:14027)

[19] S. L. Kleiman: Toward a numerical theory of ampleness. Ann. of Math. (2) 84, 293-344 (1966). MR0206009 (34:5834)

[20] P. Kleinschmidt: A classification of toric varieties with few generators. Aequationes Math. 35, 254-266 (1988). MR0954243(89f:14056)

[21] F. Knop: Über Hilberts vierzehntes Problem für Varietäten mit Kompliziertheit eins. Math. Z. 213, 33-36 (1993). MR 1217668(94b:14050)

[22] F. Knop, H. Kraft, T. Vust: The Picard group of a G-variety, in: Algebraische Transformationsgruppen und Invariantentheorie, DMV Seminar, Vol. 13, Birkhäuser, Basel (1989). MR 1044586

[23] J. Kollár: Rational curves on algebraic varieties. Springer-Verlag, Berlin (1995). MR 1440180 (98c:14001)

[24] K. Leichtweiß: Konvexe Mengen. Springer-Verlag, Berlin, Heidelberg, New York (1980). MR0586235 (81j:52001)

[25] K. Matsuki: Introduction to the Mori program. Springer-Verlag, Berlin, Heidelberg, New York (2001). MR1875410(2002m:14011)

[26] D. Mumford, J. Fogarty, F. Kirwan: Geometric invariant theory. 3rd enl. ed.. Ergebnisse der Mathematik und ihrer Grenzgebiete. Springer-Verlag, Berlin, Heidelberg, New York (1993). MR:1304906 (95m:14012)

[27] T. Oda, H. S. Park: Linear Gale Transforms and Gelfand-Kapranov-Zelevinskij Decompositions. Tôhoku Math. J. 43, 375-399 (1991). MR1117211(92d:14042)

[28] J. Świẹcicka: A combinatorial construction of sets with good quotients by an action of a reductive group. Colloq. Math. 87, 85-102 (2001). MR1812145(2001m:14069)

[29] B. Szendröi: On a conjecture of Cox and Katz. Math. Z. 240, 233-241 (2002). MR1900310 (2003c:14043)

[30] H. Whitney: Complex Analytic Varieties. Addison-Wesley Publishing Company, Inc. (1972). MR0387634 (52:8473)

[31] J. Włodarczyk: Embeddings in toric varieties and prevarieties. J. Algebr. Geom. 2, 705-726 (1993). MR1227474(94e:14070)

Mathematisches Institut, Universität Heidelberg, 69221 Heidelberg, Germany Current address: Fachbereich Mathematik und Statistik, Universität Konstanz, D-78457 Konstanz, Germany

E-mail address: Florian.Berchtold@uni-konstanz.de

Mathematisches Forschungsinstitut Oberwolfach, Lorenzenhof, 77709 OberwolfachWalke, Germany

Current address: Mathematisches Institut, Universität Tübingen, Auf der Morgenstelle 10, 72076 Tübingen, Germany

E-mail address: hausen@mail.mathematik.uni-tuebingen.de 